\title{
Nanowire and Nanocable Intrinsic Quantum Capacitances and Junction Capacitances: Results for Metal and Semiconducting Oxides
}

\author{
C. M. Krowne \\ Microwave Technology Branch, Naval Research Laboratory, Washington, DC 20375, USA \\ Correspondence should be addressed to C. M. Krowne, cliff.krowne@nrl.navy.mil \\ Received 16 August 2010; Accepted 25 October 2010 \\ Academic Editor: Xuedong Bai
}

Copyright ( $\odot 2010$ C. M. Krowne. This is an open access article distributed under the Creative Commons Attribution License, which permits unrestricted use, distribution, and reproduction in any medium, provided the original work is properly cited.

\begin{abstract}
Here we calculate the intrinsic quantum capacitance of $\mathrm{RuO}_{2}$ nanowires and $\mathrm{RuO}_{2} / \mathrm{SiO}_{2}$ nanocables (filled interiors of nanotubes, which are empty), based upon available ab initio density of states values, and their conductances allowing determination of transmission coefficients. It is seen that intrinsic quantum capacitance values occur in the aF range. Next, expressions are derived for Schottky junction and $p-n$ junction capacitances of nanowires and nanocables. Evaluation of these expressions for $\mathrm{RuO}_{2}$ nanowires and $\mathrm{RuO}_{2} / \mathrm{SiO}_{2}$ nanocables demonstrates that junction capacitance values also occur in the aF range. Comparisons are made between the intrinsic quantum and junction capacitances of $\mathrm{RuO}_{2}$ nanowires and $\mathrm{RuO}_{2} / \mathrm{SiO}_{2}$ nanocables, and between them and intrinsic quantum and junction capacitances of carbon nanotubes. We find that the intrinsic quantum capacitance of $\mathrm{RuO}_{2}$-based nanostructures dominates over its junction capacitances by an order of magnitude or more, having important implications for energy and charge storage.
\end{abstract}

\section{Introduction}

With the past work on nanowire and nanotube (or nanocable) materials, including silicon nanowires [1-4], carbon nanotubes (single and many walled) [5-19], metallic nanowires, and now nanoribbon graphene [20-29], it is not surprising that so many uses have been found for nanostructured one-dimensional (1D) objects. These uses range from employment in solid state devices like Schottky and $p-n$ diodes to transistors in electronics, to uses as catalysts, fuel cell membranes, batteries, and supercapacitors for energy applications, with solid state solar cells bridging the gap between electronic and energy uses.

Recent findings [30] for a particularly interesting metallic oxide, ruthenium dioxide $\mathrm{RuO}_{2}$, deposited as a shell on an inner silicon dioxide $\mathrm{SiO}_{2}$ core (forming a coaxial cable geometry), have shown possibilities for use as fuel cells and solar cells because of its anomalously high electronic conductivity $\left(0.5 \mathrm{~S} / \mathrm{cm}\right.$ at $0.1 \%$ volume of $\mathrm{RuO}_{2}$ in the $\mathrm{RuO}_{2} / \mathrm{SiO}_{2}$ composite when deposited as nanoclusters; conductivity range is discussed in $[31,32]$ related to its most ordered simplest crystalline form to least ordered forms), optical transparency, negligible amount of expensive atomic Ru element used $\left(0.3 \mathrm{mg}\right.$ of $\mathrm{RuO}_{2}$ per square of $\mathrm{SiO}_{2}$ paper$28 \notin$ per square), ultrahigh surface area $\left(90 \mathrm{~m}^{2}\right.$ per gram $\mathrm{RuO}_{2}$ ), anomalously high energy storage ( $>700 \mathrm{~F}$ per gram anhydrous $\mathrm{RuO}_{2}$ ), and vigorous catalytic action for water splitting. Previous uses in the bulk and nanoscale forms include Schottky barrier photovoltaics [33], field emission of nanorods [34], and thick film resistors [35]. To properly make use of $\mathrm{RuO}_{2}$, its atom scale chemistry is important in addition to electronic properties $[36,37]$.

There are other structured metal oxides which have potential for electronic applications, including cathodoluminescent $\mathrm{Ga}_{2} \mathrm{O}_{3}$ nanowires [38-42], electrochromic behavior materials $\left(\mathrm{WO}_{3}, \mathrm{MoO}_{3}, \mathrm{TiO}_{2}, \mathrm{~V}_{2} \mathrm{O}_{3}\right.$, and $\mathrm{Sb}$-doped $\mathrm{SnO}_{2}$ dispersed into inert inorganic supports) [43, 44], $\mathrm{WO}_{3}$ nanowires on $W$ [45], resistive gas-sensor materials (like $\mathrm{WO}_{3}, \mathrm{SnO}_{2}$, and $\mathrm{In}_{2} \mathrm{O}_{3}$ ) [46], and indium-tin-oxide transparent conductive materials $[47,48]$ which can be used for solar cells and LEDs, for example. Furthermore, gadolinium-based oxides $[49,50]$ may hold potential for 
ionic nanowires. Finally, nanobeams, represent quasi-onedimensional objects, smaller than characteristic grain sizes, that may be of interest, which have been realized in $\mathrm{VO}_{2}$, and are semiconductors below a critical temperature $T_{c}=68 \mathrm{C}$ with an optical bandgap $E_{g}=0.6 \mathrm{eV}$ [51].

The following four sections treat the intrinsic quantum capacitance of $\mathrm{RuO}_{2}$ nanowires (Section 2), quantum conductance and transmission coefficient of $\mathrm{RuO}_{2}$ nanowires (Section 3), quantum conductance and transmission coefficient of $\mathrm{RuO}_{2} / \mathrm{SiO}_{2}$ nanocables (Section 4), and intrinsic quantum capacitance of $\mathrm{RuO}_{2} / \mathrm{SiO}_{2}$ nanocables (Section 5). After these four sections, the next two sections cover semiconductor junction capacitances of nanocables (Section 6) and nanowires (Section 7). Section 6 is divided into Sections 6.1 and 6.2 examining, respectively, Schottkyand asymmetric-semiconductor junction capacitances of nanocables. Section 7 also is divided into Sections 7.1 and 7.2, investigating, respectively, symmetric- and asymmetricsemiconductor junction capacitances of nanocables.

Finally, the paper presents a discussion and comparison in Section 8 between the intrinsic quantum capacitances and the junction capacitances, in four parts, Sections 8.1, 8.2, 8.3, and 8.4 , focusing on respectively, junction capacitances of nanocables and nanowires, intrinsic quantum capacitances of nanocables and nanowires, comparisons between intrinsic quantum and junction capacitances of nanocables and nanowires, and electrochemical aspects in relation to the physics of nanowires and nanocables. A short conclusion follows (Section 9). Next follow two appendices, one providing details on the Green's function solution for Poisson's equation in the electrostatic limit (Appendix A), the other on modifications in the nonabrupt nanocable junction potential with distance along the longitudinal axis (Appendix B).

\section{Intrinsic Quantum Capacitance of $\mathrm{RuO}_{2}$ Nanowires}

Intrinsic quantum capacitance of nanowires (Figure 1(a)), based upon charge storage of electron carriers, calculated from the density of states (DOSs) determined from first principles quantum simulations employing the orbital structure of the crystalline system, utilizing the unit of electron charge magnitude $e$, is given in the report by Amantram and Léonard [13] and the text by Leonard [14] as

$$
C_{i}=e^{2} D_{l}\left(E_{F}\right),
$$

where $D_{l}\left(E_{F}\right)$ is the density of states at the Fermi level $E_{F}$ and the subscript $l$ on $D_{l}$ indicates that this is one dimensional density of states whose units are $\mathrm{eV}^{-1} \cdot \mathrm{nm}^{-1}$. Equation (1) may be derived by finding the added energy stored by adding electrons to the system when $E=E(k)$, finding for a single electron $\delta E=d E(k) /\left.d k\right|_{E=E_{F}} \delta k=\delta k /\left[2 \pi D\left(E_{F}\right)\right]$, using the discrete level separation $\delta k=2 \pi / L$ for a nanowire (or nanocable) of length $L$, and equating this band structure energy to the capacitive energy stored $E_{C}$, where $e=Q=C V$ giving $e^{2}=C(e V)=C E_{C}$, making capacitance per unit length equal to $C_{l}=C / L=e^{2} / L E_{C}$. Expression (1) may also be obtained by incrementing the electrostatic potential $V$

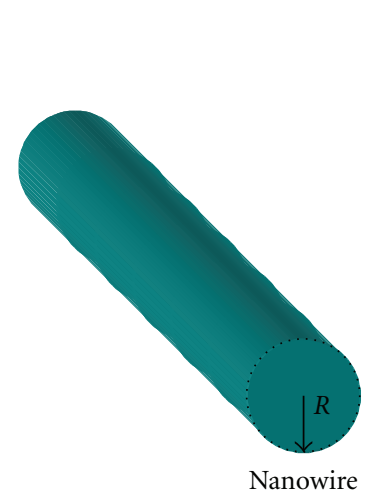

(a)

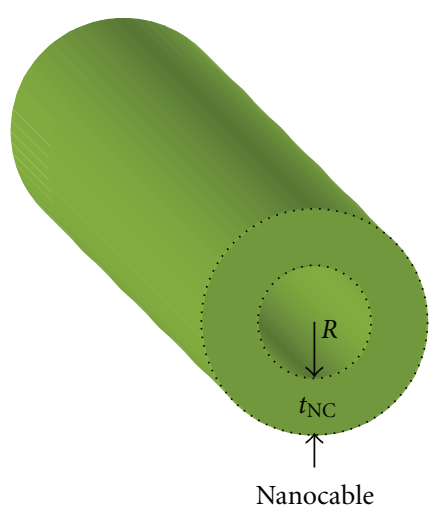

(b)
Figure 1: (a) Basic nanowire geometry. (b) Basic nanocable geometry. In the text the kernel is developed based upon the outer shell being much thicker than the inner core, namely, that $t_{\mathrm{NC}} \gg R$.

of the entire nanowire (or nanocable) to $V+\delta V$, which adds the charge $\delta Q=e \int_{-\infty}^{\infty} D_{l}(E) \delta\left[f\left(E-E_{F}\right)\right] d E$ or $\delta Q=$ $e \int_{-\infty}^{\infty} D_{l}(E)\left[f\left(E+e \delta V-E_{F}\right)-f\left(E-E_{F}\right)\right] d E$, which yields $\delta Q=e^{2} D_{l}\left(E_{F}\right) \delta V$ [because $\delta Q \approx-e \delta V \int_{-\infty}^{\infty} D_{l}(E)[d f(E-$ $\left.\left.E_{F}\right) / d E\right] d E$ which makes $\delta Q \approx-e^{2} \delta V D_{l}(E) \int_{-\infty}^{\infty}\left[d f\left(E-E_{F}\right) /\right.$ $\left.d E] d E=-e^{2} \delta V D_{l}(E) \int_{-1}^{0} d f\right]$ and $C_{i}=\delta Q / \delta V . D_{l}\left(E_{F}\right)$ may be calculated from the bulk three-dimensional (3D) density of states $D\left(E_{F}\right)$ using the following relationship:

$$
D_{l}\left(E_{F}\right)=\frac{\pi R^{2}}{V_{\text {unit-cell }}} D\left(E_{F}\right) \text {. }
$$

At the Fermi level, for $\mathrm{RuO}_{2}, D\left(E_{F}\right)$ is known to be 3.2 [52], 3.8 (rutile crystal structure [53]), 2.6 (orthorhombic crystal structure [53]), 2 [54], with [55] not providing absolute scales to extract values from but an earlier work of these authors [56] suggest a value of 1.4 (rutile structure, off of their Figure 7; [57] extracts an incorrect value of 1.7 listed in their Table 1), 2.36 [57], and 3.6 (off of Figure 4 in [58]; [57] extracts an incorrect value of 2.89 listed in their Table 1). The unit cell volume for a rutile crystal structure is $V_{\text {uni-cell }}^{\mathrm{RuO}_{2}}=a b c$ and the tetragonal values of the cell sides [34] are $a(\AA)=b(\AA)=4.45$ and $c(\AA)=3.16$, making $V_{\text {uni-cell }}^{\mathrm{RuO}_{2}}=$ $65.07078 \AA^{3} \approx 0.065071 \mathrm{~nm}^{3}$. Note that the values of $a=b$, and $c$ used are close to earlier reported values of $a=4.4909$ and $c=3.1064$ [59].

The final formula for $C_{i}$, in units of $\mathrm{aF} / \mathrm{nm}$, is given by

$$
C_{i}^{\mathrm{RuO}_{2}}=21 \cdot 1 \bar{R}^{2}(\mathrm{~nm}) D^{\mathrm{RuO}_{2}}(\# \text { states/eV } \cdot \text { unit cell }) \mathrm{aF} / \mathrm{nm} .
$$

Setting $D^{\mathrm{RuO}_{2}}\left(E_{F}\right) \approx 3 \mathrm{eV}^{-1} /$ cell, and the nanowire radius $R=1 \mathrm{~nm}$, we find that

$$
C_{i}^{\mathrm{RuO}_{2}}=63.3 \mathrm{aF} / \mathrm{nm} .
$$

This may be compared to a single-walled carbon nanotube intrinsic capacitance $C_{i}^{\text {SWCNT }}=4 e^{2} /\left(\pi \hbar v_{F}\right)=0.4 \mathrm{aF} / \mathrm{nm}$ [14]. The reason why the SWCNT formula is $R$ independent whereas the nanowire is not, is that for the nanowire larger 
$R$ means a larger cross-sectional area of atoms to include, whereas for the SWCNT, its thickness remains one atomic layer thick no matter what $R$ is. It is apparent from this calculation, that the charge storage capacity of $\mathrm{RuO}_{2}$ is over 158 times that of single-walled carbon nanotubes. This is over two orders of magnitude improvement and suggests that metal-oxide nanowires may be better for charge storage applications.

Before we can go on to calculate the intrinsic capacitance of an outer cylindrical shell of $\mathrm{RuO}_{2}$ surrounding an inner $\mathrm{SiO}_{2}$ core (coaxial cable geometry), we must study the conductivity and transmission properties of nanowires and nanocables in the next section.

\section{Quantum Conductance and Transmission Coefficient of $\mathbf{R u O}_{2}$ Nanowires}

At room temperature, the resistivity of $\mathrm{RuO}_{2}$ is given by [55]

$$
\rho_{\text {bulk }}^{\mathrm{RuO}_{2}}=34 \mu \Omega \cdot \mathrm{cm}
$$

a value considerably higher than, say a good monoatomic metal like silver, whose resistivity value is $\rho_{\text {bulk }}^{\mathrm{Ag}}=1.62 \mu \Omega \cdot \mathrm{cm}$ [60]. The value shown in (5) is in nearly perfect agreement with that in [37], who cites the value from [61], as $\rho_{\text {bulk }}^{\mathrm{RuO}_{2}}=$ $35 \mu \Omega \cdot \mathrm{cm}$. Using the slightly smaller value, we can write the conductivity as

$$
\sigma_{\text {bulk }}^{\mathrm{RuO}_{2}}=\frac{1}{\rho_{\text {bulk }}^{\mathrm{RuO}}}=29410 \Omega^{-1} \cdot \mathrm{cm}^{-1}=29410 \mathrm{~S} / \mathrm{cm} .
$$

With this conductivity value, the conductance of a $1 \mu \mathrm{m}$ long nanowire is be calculated to be

$$
\begin{aligned}
G_{\mathrm{NW}}^{\mathrm{RuO}_{2}} & =\frac{A_{\mathrm{NW}}^{\mathrm{RuO}_{2}}}{L_{\mathrm{NW}}^{\mathrm{RuO}}} \sigma_{\text {bulk }}^{\mathrm{RuO}_{2}} \\
& =9.2394 \times 10^{-6} \text { mhos }=9.2394 \mu \mathrm{S} .
\end{aligned}
$$

From this conductance, a transmission coefficient characterizing the scattering properties of the metal-oxide material can be determined. Here is how it is derived. The conductance can be expressed fairly accurately, for small applied voltage differences to the wire ends, as [10-14]

$$
G=\frac{2 e^{2}}{h} g_{\text {spin }} \sum_{m} \int_{-\infty}^{\infty} T_{m}(E)\left\{-\frac{\partial f(E)}{\partial E}\right\} d E .
$$

where $h$ is Planck's constant, $T_{m}$ is the transmission coefficient for the $m$ th mode, and $f(E)$ is the Fermi-Dirac distribution function providing the statistics for the particles under consideration. This equation can be broken into three factors, the quantum of conductance $G_{0}=2 e^{2} / h, g_{\text {spin }}$, and the summation over the integrals for each scattering mechanism, $I_{m G}$. Then (8) may be rewritten as

$$
G=G_{0} g_{\text {spin }} \sum_{m} I_{m G}
$$

where we will take the spin degeneracy to be $g_{\text {spin }}=2$, and

$$
I_{m G}=\int_{-\infty}^{\infty} T_{m}(E)\left\{-\frac{\partial f(E)}{\partial E}\right\} d E .
$$

We will assume that this integral can be represented approximately by the following product at the Fermi level:

$$
I_{m G}=T_{m}\left(E_{F}\right) f\left(E_{F}\right)
$$

and inserting this into (9) yields

$$
G=G_{0 s} f\left(E_{F}\right) \sum_{m} T_{m}\left(E_{F}\right)
$$

where we use $G_{0 s}\left(G_{0 s}=154.94 \mu \mathrm{S}\right)$ as the fundamental quantum conductance with spin degeneracy included, and write the summation of all modes of scattering as

$$
T\left(E_{F}\right)=\sum_{m} T_{m}\left(E_{F}\right)
$$

because it is the total conductance $G$ that is measured. Placing (13) into (12) allows that total transmission coefficient to be calculated as

$$
T\left(E_{F}\right)=2 \frac{G}{G_{0 s}}
$$

because the Ferm-Dirac function, $f(E)=1 /\left[1+\exp \left(E-E_{F}\right) /\right.$ $\left.k_{B} T\right]$, is simply a half when evaluated at the Fermi level. For a $1 \mathrm{~nm}$ radius nanowire of $\mathrm{RuO}_{2}$, we find from (14) that

$$
T_{\mathrm{NW}}^{\mathrm{RuO}_{2}}\left(E_{F}\right)=0.1193 \approx 0.12 .
$$

\section{Quantum Conductance and Transmission Coefficient of $\mathrm{RuO}_{2} / \mathrm{SiO}_{2}$ Nanocables}

These nanocables (see Figure 1(b)), coaxial geometry with an inner $\mathrm{SiO}_{2}$ solid cylindrical core and an outer cylindrical shell of $\mathrm{RuO}_{2}$, are described in [30], as having electronic conductivity

$$
\sigma_{\mathrm{NW}_{2} \text { cable }}^{\mathrm{RuO}_{2} / \mathrm{iO}_{2}}=0.3-0.8 \frac{\mathrm{S}}{\mathrm{cm}}, \quad 5 \mathrm{wt} \% \quad \mathrm{RuO}_{2} \text { on } \mathrm{SiO}_{2} \text {. }
$$

To use a formula like (7) to find the conductance, we need the inner core radius, outer shell radius, and their difference:

$$
\begin{gathered}
\Delta R=R_{\mathrm{RuO}_{2}}-R_{\mathrm{SiO}_{2}}=2-3 \mathrm{~nm}, \\
R_{\mathrm{SiO}_{2}}=30 \mathrm{~nm} \text { and up, } \quad R_{\mathrm{RuO}_{2}}=R_{\mathrm{SiO}_{2}}+\Delta R .
\end{gathered}
$$

Because the core-shell combination acts as a parallel resistor system, the conductance must be

$$
\begin{aligned}
G_{\mathrm{NW}^{2} \text { cable }}^{\mathrm{RuO}_{2} / \mathrm{SiO}_{2}} & =\frac{1}{L_{\mathrm{NW} \text { cable }}^{\mathrm{RuO} / \mathrm{SiO}_{2}}}\left\{A_{\mathrm{SiO}_{2}} \sigma_{\mathrm{SiO}_{2}}+A_{\mathrm{RuO}_{2}} \sigma_{\mathrm{RuO}_{2}}\right\} \\
& =\frac{A_{\mathrm{RuO}_{2}}}{L_{\mathrm{NW} \text { cable }}^{\mathrm{Ru} \mathrm{SiO}_{2}}} \sigma_{\mathrm{RuO}_{2}},
\end{aligned}
$$


where the second equality holds when the inner core is perfectly insulating, which we are assuming.

The area of the thin annulus of $\mathrm{RuO}_{2}$ clusters forming the outer shell, has an area equal to the difference between the inner and outer circular cross-sections, or

$$
A_{\mathrm{RuO}_{2}}^{\text {clust }}=2 \pi R_{a v}^{\text {cable }} \Delta R^{\text {cable }}, \quad R_{a v}^{\text {cable }}=\frac{\left(R_{\mathrm{RuO}_{2}}+R_{\mathrm{SiO}_{2}}\right)}{2} .
$$

For $\Delta R=2 \mathrm{~nm}$ and $R_{\mathrm{SiO}_{2}}=30 \mathrm{~nm}, A_{\mathrm{RuO}_{2}}^{\text {clust }}=124 \pi \mathrm{nm}^{2}$, and taking $\sigma_{\mathrm{NW} \text { cable }}^{\mathrm{RuO}_{2} / \mathrm{SiO}_{2}}=0.5 \mathrm{~S} / \mathrm{cm}$ and $L_{\mathrm{NW} \text { cable }}^{\mathrm{RuO}_{2} / \mathrm{SiO}_{2}}=1 \mu \mathrm{m}$,

$$
G_{\mathrm{NW} \text { cable }}^{\mathrm{RuO}_{2} / \mathrm{SiO}_{2}}=1.95 \times 10^{-8} \text { mhos }=0.0195 \mu \mathrm{S} .
$$

This is considerably smaller than the $\mathrm{RuO}_{2}$ nanowire result (see (7)) by a factor of

$$
\frac{G_{\mathrm{NW}}^{\mathrm{RuO}_{2}}}{G_{\mathrm{NW} \text { cable }}^{\mathrm{RuO} \mathrm{SiO}_{2}}}=474
$$

whose value is substantially smaller than an Ag nanowire by a factor of

$$
\frac{G_{\mathrm{NW}}^{\mathrm{Ag}}}{G_{\mathrm{NW}}^{\mathrm{RuO}_{2}}}=\frac{\sigma_{\mathrm{Ag}}}{\sigma_{\mathrm{RuO}_{2}}}=21
$$

How does this value compare to indium tin oxide (ITO; $5 \mathrm{wt} \% \mathrm{SnO}_{2}+95 \% \mathrm{wt} \% \mathrm{In}_{2} \mathrm{O}_{3}$ ) which is studied by Kim et al. [48]? They found that

$$
\rho_{\mathrm{ITO}}= \begin{cases}400 \mu \Omega \cdot \mathrm{cm}, & 150 \mathrm{~nm} \text { film, } \\ 200 \mu \Omega \cdot \mathrm{cm}, & 170 \mathrm{~nm} \text { film }\end{cases}
$$

so using a value of $\rho_{\text {ITO }}=300 \mu \Omega \cdot \mathrm{cm}$ for a ITO nanowire, we see that

$$
\frac{G_{\mathrm{NW}}^{\mathrm{RuO}}}{G_{\mathrm{NW}}^{\mathrm{ITO}}}=\frac{\sigma_{\mathrm{RuO}_{2}}}{\sigma_{\mathrm{ITO}}}=8.83
$$

The transmission coefficient is given by (14),

$$
T_{\mathrm{NW}_{2} \text { cable }}^{\mathrm{RuO}_{2} / \mathrm{SiO}_{2}}\left(E_{F}\right)=2 \frac{G_{\mathrm{NWcable}_{2}}^{\mathrm{RuO}_{2} / \mathrm{SiO}_{2}}}{G_{0 s}}=2.516 \times 10^{-4} .
$$

This is much smaller than that for the $\mathrm{RuO}_{2}$ nanowire, and indicates that a $\mathrm{RuO}_{2} / \mathrm{SiO}_{2}$ nanocable, with $\mathrm{RuO}_{2}$ nanoclusters having interfacial interconnects, will have transmission reduction due to those interfaces, beyond the already expected bulk-like and size-reduced geometrical scattering.

\section{Intrinsic Quantum Capacitance of $\mathrm{RuO}_{2} / \mathrm{SiO}_{2}$ Nanocables}

Following formula (1), the intrinsic quantum capacitance of $\mathrm{RuO}_{2} / \mathrm{SiO}_{2}$ nanocables is

$$
C_{i}^{\mathrm{RuO}_{2} / \mathrm{SiO}_{2}}=e^{2} D_{l}^{\mathrm{RuO}_{2} / \mathrm{SiO}_{2}}\left(E_{F}\right)
$$

with the density of states expressible for the nanocable (Figure $1(\mathrm{~b})$ ) as

$$
D_{l}^{\mathrm{RuO}_{2} / \mathrm{SiO}_{2}}\left(E_{F}\right)=\frac{2 \pi R_{a v}^{\text {cable }} \Delta R^{\text {cable }}}{V_{\text {unit-cell }}^{\mathrm{RuO}_{2}}} D^{\mathrm{RuO}_{2} / \mathrm{SiO}_{2}}\left(E_{F}\right) .
$$

Again, inserting (27) into (26), we obtain a formula similar to $(3)$,

$$
\begin{array}{r}
C_{i}^{\mathrm{RuO}_{2} / \mathrm{SiO}_{2}}=14.262 \bar{R}_{a v}^{\mathrm{cable}}(\mathrm{nm}) \Delta R^{\text {cable }} D^{\mathrm{RuO}_{2} / \mathrm{SiO}_{2}} \\
\text { (\# states } / \mathrm{eV} \cdot \text { unit cell) } \mathrm{aF} / \mathrm{nm} .
\end{array}
$$

Here, $D^{\mathrm{RuO}_{2} / \mathrm{SiO}}$ can be estimated by

$$
D^{\mathrm{RuO}_{2} / \mathrm{SiO}_{2}}\left(E_{F}\right)=\frac{G_{\mathrm{NW} \text { cable }}^{\mathrm{RuO}_{2} / \mathrm{SiO}_{2}}}{G_{\mathrm{NW}}^{\mathrm{RuO}}} D^{\mathrm{RuO}_{2}}\left(E_{F}\right) .
$$

Referring back to Section 2 for $D^{\mathrm{RuO}_{2}}$, and to (21) for the conductance ratio, $D^{\mathrm{RuO}_{2} / \mathrm{SiO}_{2}}$ can be obtained and (28) evaluated for $C_{i}^{\mathrm{RuO}_{2} / \mathrm{SiO}_{2}}$ as

$$
C_{i}^{\mathrm{RuO}_{2} / \mathrm{SiO}_{2}}=5.60 \mathrm{aF} / \mathrm{nm}
$$

This result is over an order of magnitude lower compared to the $\mathrm{RuO}_{2}$ nanowire capacitance found in (4), not a totally unexpected result.

One might wonder what the value of $C_{i}^{\mathrm{RuO}_{2} / \mathrm{SiO}_{2}}\left(\Delta R^{\text {cable }}=\right.$ $\left.t_{\mathrm{CNT}}\right)$ might be if the $\mathrm{RuO}_{2} / \mathrm{SiO}_{2}$ nanocable had the same cross-sectional area as a single-walled carbon nanotube. Using the SWCNT thickness $t_{\mathrm{CNT}}=a_{\text {orbital extent }} \approx 2 a_{\mathrm{C}-\mathrm{C}}=2$ $(1.42 \AA=0.142 \mathrm{~nm})$, consistent with Mintmire and White [5] and Leonard [14], the area of a $R=1 \mathrm{~nm}$ radius CNT ring or annulus is

$$
A_{\mathrm{CNT}}=2 \pi R t_{\mathrm{CNT}} \approx 4 \pi a_{\mathrm{C}-\mathrm{C}} R=0.568 \pi \mathrm{nm}^{2} .
$$

Equation (31) allows us to express $C_{i}^{\mathrm{RuO}_{2} / \mathrm{SiO}_{2}}\left(\Delta R^{\text {cable }}=t_{\mathrm{CNT}}\right)$ as

$$
\begin{aligned}
C_{i}^{\mathrm{RuO}_{2} / \mathrm{SiO}_{2}}\left(\Delta R^{\text {cable }}=t_{\mathrm{CNT}}\right) & =\frac{A_{\mathrm{CNT}}}{A_{\mathrm{RuO}_{2}}} C_{i}^{\mathrm{RuO}_{2} / \mathrm{SiO}_{2}} \\
& =0.0256 \mathrm{aF} / \mathrm{nm}
\end{aligned}
$$

which is an order of magnitude smaller than $C_{i}^{\text {SWCNT }}=$ $0.4 \mathrm{aF} / \mathrm{nm}$ for the SWCNT. This result is not entirely unexpected, since the electron conduction mediated by the orbitals perpendicular to the plane of the carbon nanotube cylindrical wall, whose physical extent is given by $a_{\text {orbital extent }}$, is known to be extremely large, quasiballistic in fact.

Because we know the measured value of the capacitance per gram of the $\mathrm{RuO}_{2} / \mathrm{SiO}_{2}$ nanocables, $C_{g}^{\mathrm{RuO}_{2} / \mathrm{SiO}_{2}}=$ $700 \mathrm{~F} / \mathrm{gm}$, it is possible, if we assume all this capacitance comes from the intrinsic quantum capacitance, and no junction capacitances to be discussed in the next sections, to find the effective density of $\mathrm{RuO}_{2}$ in the $\mathrm{RuO}_{2} / \mathrm{SiO}_{2}$ nanocables. Therefore, setting $C_{i, g}^{\mathrm{RuO}_{2} / \mathrm{SiO}_{2}}=C_{g}^{\mathrm{RuO}_{2} / \mathrm{SiO}_{2}}, C_{i}^{\mathrm{RuO} /} / \mathrm{SiO}_{2}$ given in (30) times the overall total length $L$, may be equated to 
$C_{i, g}^{\mathrm{RuO}_{2} / \mathrm{SiO}_{2}}$ times the total volume of the nanocables times its mass density $\rho_{\text {cable }}^{\mathrm{RuO}_{2}}$, yielding

$$
C_{i}^{\mathrm{RuO}_{2} / \mathrm{SiO}_{2}}=C_{i, g}^{\mathrm{RuO}_{2} / \mathrm{SiO}_{2}} 2 \pi R_{a v}^{\text {cable }} \Delta R^{\text {cable }} \rho_{\text {cable }}^{\mathrm{RuO}_{2}} .
$$

Solving for $\rho_{\text {cable }}^{\mathrm{RuO}_{2}}$

$$
\rho_{\text {cable }}^{\mathrm{RuO}_{2}}=\frac{C_{i}^{\mathrm{RuO}_{2} / \mathrm{SiO}_{2}}}{C_{i, g}^{\mathrm{RuO}_{2} / \mathrm{SiO}_{2}}} \frac{1}{2 \pi R_{a v}^{\text {cable }} \Delta R^{\text {cable }}}
$$

which when evaluated using the available capacitances, gives the remarkable density

$$
\rho_{\text {cable }}^{\mathrm{RuO}_{2}}=0.02052 \mathrm{gm} / \mathrm{cc} \text {. }
$$

Thus, the density reduction of $\mathrm{RuO}_{2}$ in the nanocable structure, allowing the high capacitive energy storage, is

$$
\frac{\rho_{\mathrm{cable}}^{\mathrm{RuO}_{2}}}{\rho_{\mathrm{cry}}}=340
$$

using the known value for rutile (tetragonal) crystalline structured $\mathrm{RuO}_{2}$.

\section{Semiconductor Junction Capacitances of Nanocables}

As mentioned in Section 1, there may be metallic oxides, which when properly doped, that may act as semiconductors. We already know that $\mathrm{Ga}_{2} \mathrm{O}_{3}$ nanowires [38-42], display cathodoluminescence. These oxides have a large bandgap, experimentally determined to be $E_{g}=4.8 \mathrm{eV}$ [38], whereas the theoretically determined value is about $5.8 \mathrm{eV}$ [39]. When doped with $\mathrm{Sn}$, a deep donor level $E_{d}=0.96 \mathrm{eV}$ below the conduction band arises [38], sufficient for allowing the measured cathodoluminescent properties. Because $k_{B} T=$ $0.026 \mathrm{eV}$ at room temperature, this will not be a material useful for ordinary semiconducting applications. However, the metallic oxide $\mathrm{Ga}_{2} \mathrm{O}_{3}$, and others such as $\mathrm{WO}_{3}, \mathrm{MoO}_{3}, \mathrm{TiO}_{2}$, $\mathrm{V}_{2} \mathrm{O}_{3}, \mathrm{SnO}_{2}, \mathrm{In}_{2} \mathrm{O}_{3}$, and $\mathrm{VO}_{2}$, and with other stoichiometric atomic combinations more favorable for obtaining suitable bandgaps, with available donor or acceptor species, may be found. Finally, like the metallic $\mathrm{RuO}_{2} / \mathrm{SiO}_{2}$ nanocables studied in Section 4, there may be semiconducting analogs.

In the next subsection, we will first look at one of the simplest cases, the junction between a planar metal contact and a semiconductor $n$-type nanocable. For that Schottky junction, its junction potential difference as a function of its $n$-type depletion width will be found, and from it the capacitance. After that, the much more complicated, but general case of an abrupt asymmetric $p-n$ semiconductor nanocable junction will be addressed in the second subsection. Here the junction potential difference as a function of its $p$ - and $n$-type depletion widths will be found, and for the two limiting cases of an infinitely high $p$-type doping density and symmetric doping densities, capacitances will be determined.

The following section, then, addresses nanowire junctions.
6.1. Schottky-Semiconductor Junction Capacitance of Nanocables. The nanocable potential functions can be found by an integral expression over a volume which accounts for nanocable annulus, radius, and length. For examination of the potential along the longitudinal axis of the cable, given a charge distribution $\rho(r, \phi, z)$ in the junction region between a metal contact and an $n$-doped thin annulus region (Figure 2(a)), this Schottky junction potential can be found [62]

$$
\begin{aligned}
& \varphi_{\text {annulus }}(0,0, z) \\
& =\frac{1}{\varepsilon_{a v}} \int_{-\infty}^{\infty} d z^{\prime} \int_{0}^{R} s d s \int_{0}^{2 \pi} d \phi \frac{\rho\left(s, \phi, z^{\prime}\right)}{\sqrt{s^{2}+\left(z-z^{\prime}\right)^{2}}} \\
& =\frac{2 \pi}{\varepsilon_{a v}} \int_{-\infty}^{\infty} d z^{\prime} \int_{0}^{R} s \frac{\rho\left(s, z^{\prime}\right) d s}{\sqrt{s^{2}+\left(z-z^{\prime}\right)^{2}}} \\
& \quad \approx \frac{2 \pi}{\varepsilon_{a v}} \int_{-\infty}^{\infty} d z^{\prime} \frac{R \rho\left(z^{\prime}\right) t_{\mathrm{NC}}}{\sqrt{R^{2}+\left(z-z^{\prime}\right)^{2}}}
\end{aligned}
$$

leading to the kernel (or Green's function)

$$
K\left(z, z^{\prime}\right)=\frac{2 \pi R t_{\mathrm{NC}}}{\varepsilon_{\mathrm{NC}}} \frac{1}{\sqrt{R^{2}+\left(z-z^{\prime}\right)^{2}}} .
$$

Electrostatic Green's function basis for (37) and (38) can be found in [63], for example, and is discussed in Appendix A (Green's function solution of Poisson's equation for electrostatic approach to field solution).

Potential due to the $n$-side of a metal planar contact$n$ nanocable junction is given, using (38), by [64, page 81 , $2.261, c>0]$

$$
\begin{aligned}
V_{n}^{\text {ring, cable }}(z)= & \int_{0}^{W} K\left(z, z^{\prime}\right) \rho\left(z^{\prime}\right) d z^{\prime} \\
& =\rho \frac{2 \pi R t_{\mathrm{NC}}}{\varepsilon_{\mathrm{NC}}} \int_{0}^{W} \frac{d z^{\prime}}{\sqrt{R^{2}+\left(z-z^{\prime}\right)^{2}}} \\
& =\rho \frac{2 \pi R t_{\mathrm{NC}}}{\varepsilon_{\mathrm{NC}}} \int_{-z}^{W-z} \frac{d \bar{z}^{\prime}}{\sqrt{R^{2}+\bar{z}^{\prime 2}}} \\
& =\left.\rho \frac{2 \pi R t_{\mathrm{NC}}}{\varepsilon_{\mathrm{NC}}} \ln \left[2\left(\sqrt{R^{2}+\bar{z}^{\prime 2}}+\bar{z}^{\prime}\right)\right]\right|_{-z} ^{W-z} \\
& =\rho \frac{2 \pi R t_{\mathrm{NC}}}{\varepsilon_{\mathrm{NC}}} \ln \left[\frac{\sqrt{R^{2}+(W-z)^{2}}+W-z}{\sqrt{R^{2}+z^{2}}-z}\right]
\end{aligned}
$$

caused by the charge density depletion separation (assumed abrupt for simplicity nonabruptness is addressed in Appendix B) in the annulus volume

$$
\rho_{r s}(z)= \begin{cases}\rho, & 0<z<W \\ -\frac{W}{\delta} \rho, & -\delta<z<0, \\ 0, & z<-\delta \text { or } \quad z>W .\end{cases}
$$




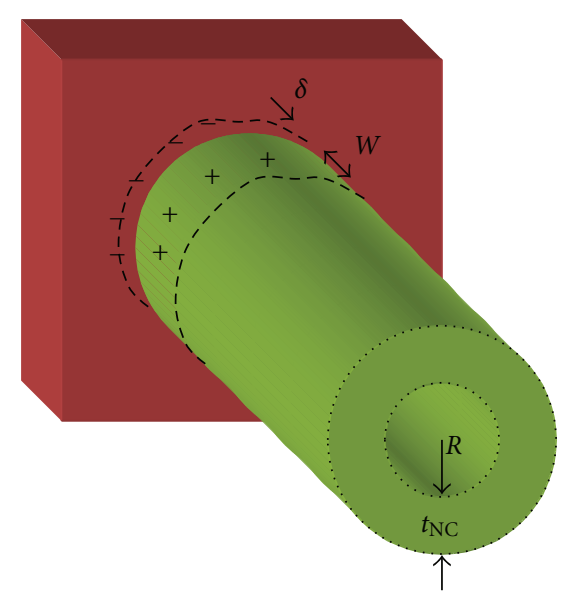

Planar metal- $n$ nanocable junction

(a)

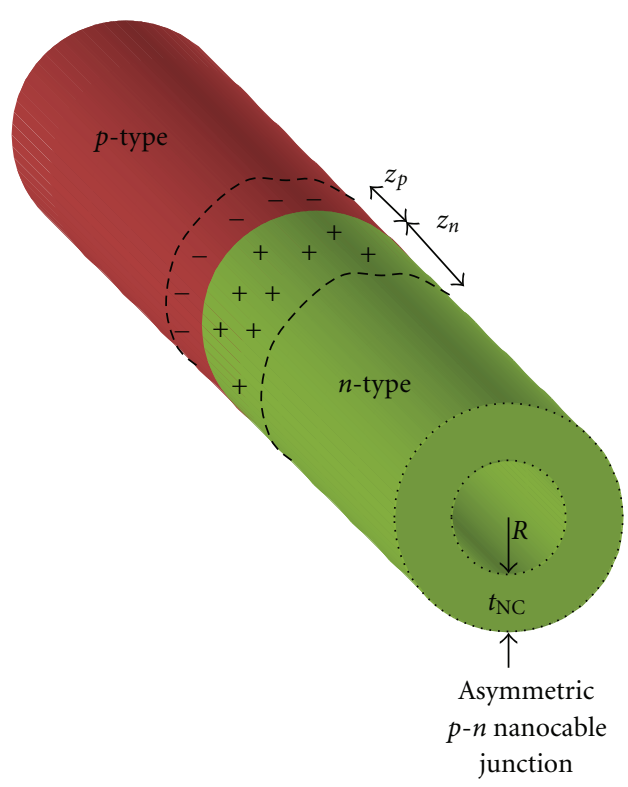

(b)

FIGURE 2: (a) Schottky junction between a planar metal contact and a semiconductor $n$-type nanocable. (b) Asymmetric semiconductor $p$ - $n$ nanocable junction. Two limits are examined in detail, the case when $\rho_{p} \rightarrow \infty$, the infinitely asymmetric case, and the exactly symmetric case, when $\rho_{p}=\rho_{n}$.

Here $\rho=e N_{d}$. Equation (40) has incorporated the condition of charge neutrality,

$$
A_{\mathrm{NC}} \int_{0}^{W} \rho\left(z^{\prime}\right) d z^{\prime}=-A_{\mathrm{NC}} \int_{-\delta}^{0} \rho\left(z^{\prime}\right) d z^{\prime} .
$$

For the image charge in the metal (which is negative), its potential contribution is

$$
\begin{aligned}
& V_{\text {metal }}^{\text {ring, cable }}(z) \\
& \quad=\int_{-\delta}^{0} K\left(z, z^{\prime}\right) \rho\left(z^{\prime}\right) d z^{\prime} \\
& =-\rho \frac{W}{\delta} \frac{2 \pi R t_{\mathrm{NC}}}{\varepsilon_{\mathrm{NC}}} \int_{-\delta}^{0} \frac{d z^{\prime}}{\sqrt{R^{2}+\left(z-z^{\prime}\right)^{2}}} \\
& =-\rho \frac{W}{\delta} \frac{2 \pi R t_{\mathrm{NC}}}{\varepsilon_{\mathrm{NC}}} \int_{-\delta-z}^{-z} \frac{d \bar{z}^{\prime}}{\sqrt{R^{2}+\bar{z}^{\prime 2}}} \\
& =-\left.\rho \frac{W}{\delta} \frac{2 \pi R t_{\mathrm{NC}}}{\varepsilon_{\mathrm{NC}}} \ln \left[2\left(\sqrt{R^{2}+\bar{z}^{\prime 2}}+\bar{z}^{\prime}\right)\right]\right|_{-\delta-z} ^{-z} \\
& =-\rho \frac{W}{\delta} \frac{2 \pi R t_{\mathrm{NC}}}{\varepsilon_{\mathrm{NC}}} \ln \left[\frac{\sqrt{R^{2}+z^{2}}-z}{\sqrt{R^{2}+(\delta+z)^{2}}-\delta-z}\right] .
\end{aligned}
$$

The total potential along the nanocable length $z$ will then be a superposition of both the donor depletion and metal image ring charge potentials in (39) and(42)

$$
V_{\mathrm{NC}}=V_{\text {metal }}^{\text {ring, cable }}+V_{n}^{\text {ring,cable }}
$$

or

$$
\begin{aligned}
V_{\mathrm{NC}}= & \rho \frac{2 \pi R t_{\mathrm{NC}}}{\varepsilon_{\mathrm{NC}}} \\
& \times\left\{-\frac{W}{\delta} \ln \left[\frac{\sqrt{R^{2}+z^{2}}-z}{\sqrt{R^{2}+(\delta+z)^{2}}-\delta-z}\right]\right. \\
& \left.+\ln \left[\frac{\sqrt{R^{2}+(W-z)^{2}}+W-z}{\sqrt{R^{2}+z^{2}}-z}\right]\right\} .
\end{aligned}
$$

We note that for large $z(z \gg W, \delta)$,

$$
V_{\mathrm{NC}}=\rho \frac{2 \pi R t_{\mathrm{NC}}}{\varepsilon_{\mathrm{NC}}}\left\{1+\frac{\delta}{W}\right\}\left(\frac{W}{z}\right)^{2} .
$$

The capacitance must be given by

$$
C_{\mathrm{NC}}= \pm \frac{d Q_{\mathrm{NC}}}{d V_{\mathrm{bias}}}
$$

where the differential element is taken of

$$
Q_{\mathrm{NC}}=\rho 2 \pi R_{a v}^{\mathrm{NC}} \Delta R^{\mathrm{NC}} W
$$

and the bias voltage across the junction is related to the junction voltage $V_{j}^{\mathrm{NC}}$ by

$$
V_{j}^{\mathrm{NC}}=V_{b i} \pm \frac{V_{\mathrm{bias}}-\alpha_{\mathrm{NC}} k_{B} T}{e},
$$

where sign of $V_{\text {bias }}$ is associated with, respectively, reverse or forward bias and the last term is an approximate correction 
due to the mobile majority carrier spatial distribution tail, discussed in Sze with related references [65]. The tail correction is based upon bulk arguments, and it is expected to be somewhat different by a proportional factor $\alpha_{\mathrm{NC}}$. The junction voltage, enlisting (44), is

$$
\begin{aligned}
V_{j}^{\mathrm{NC}}= & V_{\mathrm{NC}}(W)-V_{\mathrm{NC}}(-\delta) \\
= & \rho \frac{2 \pi t_{\mathrm{NC}}}{\varepsilon_{\mathrm{NC}}} \\
& \times\left\{-\frac{W}{\delta} \ln \left[\frac{\sqrt{R^{2}+W^{2}}-W}{\sqrt{R^{2}+(\delta+W)^{2}}-\delta-W}\right]\right. \\
& +\ln \left[\frac{R}{\sqrt{R^{2}+W^{2}}-W}\right] \\
& +\frac{W}{\delta} \ln \left[\frac{\sqrt{R^{2}+\delta^{2}}+\delta}{R}\right] \\
& \left.-\ln \left[\frac{\sqrt{R^{2}+(W+\delta)^{2}}+W+\delta}{\sqrt{R^{2}+\delta^{2}}+\delta}\right]\right\} .
\end{aligned}
$$

For the case where $W \gg \delta$,

$$
\left.V_{j}^{\mathrm{NC}}\right|_{W \gg \delta}=\rho \frac{2 \pi t_{\mathrm{NC}}}{\varepsilon_{\mathrm{NC}}}\left\{\frac{2 W}{R}-\frac{1}{\sqrt{1+(R / W)^{2}}}\right\} .
$$

Because we would expect $W / R \gg 1$ for nanowires and nanocables, taking the limiting form of (50) for $W / R \rightarrow \infty$ is reasonable and yields

$$
\left.V_{j}^{\mathrm{NC}}\right|_{\substack{W \gg \delta \rightarrow 0 \\ W / R \gg 1}} \approx \rho \frac{4 \pi t_{\mathrm{NC}}}{\varepsilon_{\mathrm{NC}}}\left(\frac{W}{R}\right)
$$

allowing $W$ to be expressed as

$$
\left.W_{\mathrm{NC}}\right|_{W / R \gg 1 \gg \delta / R \rightarrow 0}=\frac{\left.\varepsilon_{\mathrm{NC}} V_{j}^{\mathrm{NC}}\right|_{W \gg \delta \rightarrow 0, W / R \gg 1}}{e N_{d}} \frac{R}{4 \pi t_{\mathrm{NC}}} .
$$

For an unbiased device, the junction voltage may be replaced by the built-in voltage, giving

$$
\left.W_{b i}^{\mathrm{NC}}\right|_{W / R \gg 1 \gg \delta / R \rightarrow 0}=\frac{\varepsilon_{\mathrm{NC}} V_{b i}}{e N_{d}} \frac{R}{4 \pi t_{\mathrm{NC}}},
$$

where the $4 \pi$ part is simply indicative that we are using cgs units.

The single permittivity $\varepsilon_{\mathrm{NC}}$ characterizing the nanocable takes into account the field penetration from the semiconducting shell into both the dielectric core and the outside medium, often air but it could be another surrounding dielectric. It might be estimated by

$$
\varepsilon_{\mathrm{NC}} \approx \frac{w_{\text {diel }}^{\text {inner }} \varepsilon_{\text {diel }}^{\text {inner }}+w_{\text {shell }}^{\text {semi }} \varepsilon_{\text {shell }}^{\text {semi }}+w_{\text {diel }}^{\text {outer }} \varepsilon_{\text {diel }}^{\text {outer }}}{w_{\text {diel }}^{\text {inner }}+w_{\text {shell }}^{\text {semi }}+w_{\text {diel }}^{\text {outer }}} .
$$

Equation (53) enables the use of a single permittivity, which was the basis of developing a tractable kernel or electrostatic
Green's function approach. Without this assumption, a much more complicated field matching approach must be utilized, involving continuity conditions at cylindrical interfaces implying Bessel function type solutions [66-71]. It should be noted that a more accurate form of $C_{\mathrm{NC}}$ can be found by using the equality in (50) and taking the derivative of both sides of that equation with respect to $V_{\text {bias }}$, solving for $d W / d V_{\text {bias }}$, and inserting that into the capacitive expression of (46). When this is done, one finds that

$$
\begin{aligned}
& C_{\mathrm{NC}}^{\text {Schottky }} \\
& =\frac{R \varepsilon_{\mathrm{NC}}}{2} \\
& \quad \times\left[1-\frac{1}{2} \frac{1}{\sqrt{1+(W / R)^{2}}}+\frac{1}{2}\left(\frac{W}{R}\right)^{2} \frac{1}{\left[1+(W / R)^{2}\right]^{3 / 2}}\right]^{-1} .
\end{aligned}
$$

To obtain $C_{\mathrm{NC}}^{\text {Schottky }}$ in terms of $V_{\mathrm{bi}}$, set $V_{j}^{\mathrm{NC}}=V_{b i}$ on the lefthand side of $(50)$

$$
V_{\mathrm{bi}}=\rho \frac{2 \pi t_{\mathrm{NC}}}{\varepsilon_{\mathrm{NC}}}\left\{\frac{2 W}{R}-\frac{1}{\sqrt{1+(R / W)^{2}}}\right\}
$$

and solve for $W\left(V_{\mathrm{bi}}\right)$, and insert this into (55).

Taking the derivative of (52) with respect to $V_{\text {bias, }}$ and inserting into (46), the capacitance is

$$
C_{\mathrm{NC}}^{\text {Schottky }}=\frac{1}{2} R \varepsilon_{\mathrm{NC}}
$$

which is amazingly independent of explicit doping dependence. However, (53) shows that the depletion width does have this dependence. If we evaluate the capacitance for radaii typical of a carbon nanotube, say at $R=1 \mathrm{~nm}$, then

$$
C_{\mathrm{NC}}^{\text {Schottky }}=C_{\mathrm{CNT}}^{\text {Schottky }}=0.0563 \mathrm{aF}, \quad \varepsilon_{\mathrm{CNT}}=1.0 .
$$

Equation (58) assumes most of the volume is air. If instead, the CNT was surrounded by a high dielectric constant like water with $\varepsilon_{\mathrm{H}_{2} \mathrm{O}}=80.1$ at $20 \mathrm{C}$, then $C_{\mathrm{NC}}^{\text {Schottky }}=C_{\mathrm{CNT}}^{\text {Schottky }}=$ $4.5 \mathrm{aF}$.

For our $\mathrm{RuO}_{2} / \mathrm{SiO}_{2}$ nanocable dimensions, replacing $R \rightarrow R_{a v}^{\text {cable }}=31 \mathrm{~nm}$ makes the capacitance

$$
C_{\mathrm{NC}, \text { Schottky }}^{\mathrm{RuO}_{2} / \mathrm{SiO}_{2}}=6.44 \mathrm{aF}, \quad \varepsilon_{\mathrm{NT}}=3.69
$$

using a relative permittivity $\varepsilon_{\mathrm{NC}}=3.69$ which is a compromise between the core $\varepsilon_{\mathrm{SiO}_{2}}=2.15$ at $546.1 \mathrm{~nm}$ [72], and that of $\mathrm{RuO}_{2}$ with $\varepsilon_{\mathrm{RuO}_{2}}(E>2 \mathrm{eV}) \leq 3,4<\left|\varepsilon_{\mathrm{RuO}_{2}}(E=1 \mathrm{eV})\right|<$ 6 , and $2<\varepsilon_{\mathrm{RuO}_{2}, \infty}<3$, where $\varepsilon_{\mathrm{RuO}_{2}, \infty}$ is the high frequency dielectric constant $[57,73,74]$. This choice will be utilized throughout the remainder of the paper for the nanocable.

One may wonder what happens to capacitance, if in formula (51) the power of the $(W / R)$ factor was 2 , not 1 . (This actually happens for Schottky nanowires-see Section 7.2, and here occurs by dropping the second term in (50).) This 
makes $\left.W_{b i}^{\mathrm{NC}}\right|_{W / R \gg 1 \gg \delta / R \rightarrow 0}=\sqrt{\left(R \varepsilon_{\mathrm{NC}} V_{b i}\right) /\left[4 \pi t_{\mathrm{NC}} e N_{d}\right]}$, and leads to the capacitance per unit area of

$$
\begin{aligned}
C_{\mathrm{NC}}^{u a} & = \pm \frac{1}{A_{\mathrm{NC}}} \frac{d Q_{\mathrm{NC}}}{d V_{\mathrm{bias}}} \\
& = \pm e N_{d} \frac{d W\left(V_{\mathrm{bias}}\right)}{d V_{\mathrm{bias}}} \\
& =\sqrt{\frac{e \varepsilon_{\mathrm{NC}} N_{d}}{2}}\left(V_{b i} \pm \frac{V_{\text {bias }}-\alpha_{\mathrm{NC}} k_{B} T}{e}\right)^{-1 / 2} \sqrt{\frac{R}{8 \pi t_{\mathrm{NC}}}} \\
& =\frac{\varepsilon_{\mathrm{NC}}}{\sqrt{2} L_{D}^{\mathrm{NC}}}\left(\beta V_{b i} \pm \beta V_{\mathrm{bias}}-\alpha_{\mathrm{NC}}\right)^{-1 / 2}
\end{aligned}
$$

and looks like the classical bulk form with planar junction modified by the last factor in the third line or has a newly defined Debye length $L_{D}^{\mathrm{NC}}$, given by

$$
L_{D}^{\mathrm{NC}}=L_{D}^{\text {bulk }} \sqrt{\frac{8 \pi t_{\mathrm{NC}}}{R}}, \quad L_{D}^{\text {bulk }}=\sqrt{\frac{\varepsilon_{\mathrm{NC}}}{e N_{d} \beta}}, \quad \beta=\frac{e}{k_{B} T} .
$$

Let us evaluate $C_{\mathrm{NC}}$ for a carbon nanotube, using (60), noting that for small bias voltages and a built-in voltage $V_{\mathrm{bi}}=$ $0.42 \mathrm{~V}$ typical of a SWCNT, $\alpha_{\mathrm{NC}} \approx 1$, at room temperature, the square root factor reduces to $1 / \sqrt{V_{\mathrm{bi}}}$. Set $R=1 \mathrm{~nm}$ and $t_{\mathrm{NC}}=t_{\mathrm{NT}}=0.284 \mathrm{~nm}, A_{\mathrm{NC}}=A_{\mathrm{CNT}}=2 \pi R t_{\mathrm{NT}}$, giving from (60)

$$
C_{\mathrm{NC}}^{\mathrm{Schottky}}=R \sqrt{R t_{\mathrm{NC}}} \sqrt{\frac{e \varepsilon_{\mathrm{NC}} N_{d} \pi}{4 V_{\mathrm{bi}}}}=\frac{R^{2}}{4} \frac{\varepsilon_{\mathrm{NC}}}{W_{\mathrm{bi}}^{\mathrm{NC}}}
$$

(last equality in (62) follows from (53), and it yields the form $C_{\mathrm{NC}, u a}^{\text {Schottky }}=\left(\varepsilon_{\mathrm{NC}} / W_{b i}^{\mathrm{NC}}\right)\left(R / t_{\mathrm{NC}}\right)(1 / 2)(1 /[4 \pi])$, with a typical bulk like factor [65], modified by the nanocable parameters). Equation (62) arises if the first term in the $(W / R)^{2}$ contribution of (50) is dropped. Anyway, using (62) gives for the CNT capacitance

$$
\begin{gathered}
C_{\mathrm{NC}}^{\text {Schottky }}=C_{\mathrm{CNT}}=0.033 \mathrm{aF}, \\
N_{d}=5 \times 10^{20} / \mathrm{cc}, \quad \varepsilon_{\mathrm{NT}}=1.0
\end{gathered}
$$

which corresponds to a fraction $f=10^{-2}$ of $\mathrm{C}$ atoms contributing electrons $\left(N_{d}=f N_{\mathrm{CNT}}=0.5 / \mathrm{nm}^{3}\right)$, if the number of atoms in a volumetric sense is approximated as $N_{\mathrm{CNT}}=$ $5 \times 10^{22} / \mathrm{cc}$, a value consistent with Avogadro's number and other atomic densities [75]. Using the volume in a $l_{\mathrm{CNT}}=1 \mathrm{~nm}$ length, $V_{\mathrm{CNT}}=l_{\mathrm{CNT}} A_{\mathrm{CNT}}=1.784 \mathrm{~nm}^{3}$, the number of doped atoms is $N_{\mathrm{CNT}}^{\text {doped atoms }}=V_{\mathrm{CNT}} N_{d}=$ 0.892 atoms, which is quite believable. An even more accurate way to estimate this number is to use the unwrapped flat graphene hexagonal unit cell size determined in terms of the C-C distance found in (31), $A_{\text {hex }}=(3 / 2) \sqrt{3} a_{\mathrm{C}-\mathrm{C}^{2}}$, the number of carbon atoms in this cell $N_{\text {hex }}=2$, and find the volume per atom as

$$
\begin{aligned}
V_{\text {atom }} & =\frac{A_{\text {hex }}}{N_{\text {hex }}} t_{\mathrm{CNT}}=\frac{A_{\text {hex }}}{2} 2 a_{\mathrm{C}-\mathrm{C}} \\
& =\frac{3}{2} \sqrt{3} a_{\mathrm{C}-\mathrm{C}}{ }^{3}=7.439 \times 10^{-3} \mathrm{~nm}^{3}
\end{aligned}
$$

corresponding to $N_{\mathrm{CNT}}=1 / V_{\text {atom }}=1.344 \times 10^{23} / \mathrm{cc}$ and $N_{d}=f N_{\mathrm{CNT}}=1.34 / \mathrm{nm}^{3}$. The number of atoms in the solid annulus of the CNT then would be $N_{\mathrm{CNT}}^{\text {atoms }}=V_{\mathrm{CNT}} / V_{\text {atom }}=$ 239.82, making $N_{\mathrm{CNT}}^{\text {doped atoms }}=f N_{\mathrm{CNT}}^{\text {atoms }}=2.40$.

For our $\mathrm{RuO}_{2} / \mathrm{SiO}_{2}$ nanocables dimensions, replacing $R \rightarrow R_{a v}^{\text {cable }}=31 \mathrm{~nm}$ and $t_{\mathrm{NC}} \rightarrow \delta R^{\text {cable }}=2 \mathrm{~nm}$ in (62), we find

$$
C_{\mathrm{NC}, \text { Schottky }}^{\mathrm{RuO}_{2} / \mathrm{SiO}_{2}}=60.46 \mathrm{aF}, \quad N_{d}=5 \times 10^{20} / \mathrm{cc}, \quad \varepsilon_{\mathrm{NT}}=3.69
$$

using the same doping density we had for the carbon nanotube. That may not be entirely reasonable, and using a value two orders of magnitude lower for $N_{d}$ yields

$$
C_{\mathrm{NC}, \text { Schottky }}^{\mathrm{RuO}_{2} / \mathrm{SiO}_{2}}=6.05 \mathrm{aF}, \quad N_{d}=5 \times 10^{18} / \mathrm{cc}, \quad \varepsilon_{\mathrm{NT}}=3.69 .
$$

The $N_{d}$ employed in the last calculation is commonly seen for ordinary semiconductors, and avoids the high value enlisted in the CNT calculation, which as we had seen may even be higher, approaching $N_{d}=1.344 \times 10^{21} / \mathrm{cc}$ for $f=10^{-2}$ fractional doping. (Even $f=10^{-4}$ could yield $N_{d}=1.344 \times$ $10^{19} /$ cc.)

What we learn from examining the capacitance results of (58) and (59) which rely upon a linear junction voltagedepletion width relationship, and (63), (65), and (66) which uses a planar bulk-like quadratic behavior, is that the values are quite sensitive to the details of the nanostructure geometry and associated derivation details.

Effect of evaluating $C_{\mathrm{NC}}^{\text {Schottky }}$ using (57), the simpler Schottky junction capacitance formula, versus using (55), is shown in Figure 3, where the normalized capacitance $C_{\mathrm{NC}}^{\text {Schottky }} /\left(R \varepsilon_{\mathrm{NC}}\right)$ is plotted against the ratio $W / R$. Also, formula (62) resulting from dropping a term, is also plotted. It is seen that agreement between (55) and (57) becomes very close as $W / R \rightarrow 10$, whereas for $W / R=1$, the error is noticeable at $17.7 \%$. Formula (62) has a declining trend, but is way off in magnitude from the accurate expression (55).

6.2. Asymmetric-Semiconductor $p-n$ Junction Capacitance of Nanocables. For the asymmetric abrupt $p-n$ junction (Figure 2(b)), unequal doping occurs in the $p$ - and $n$-sides 


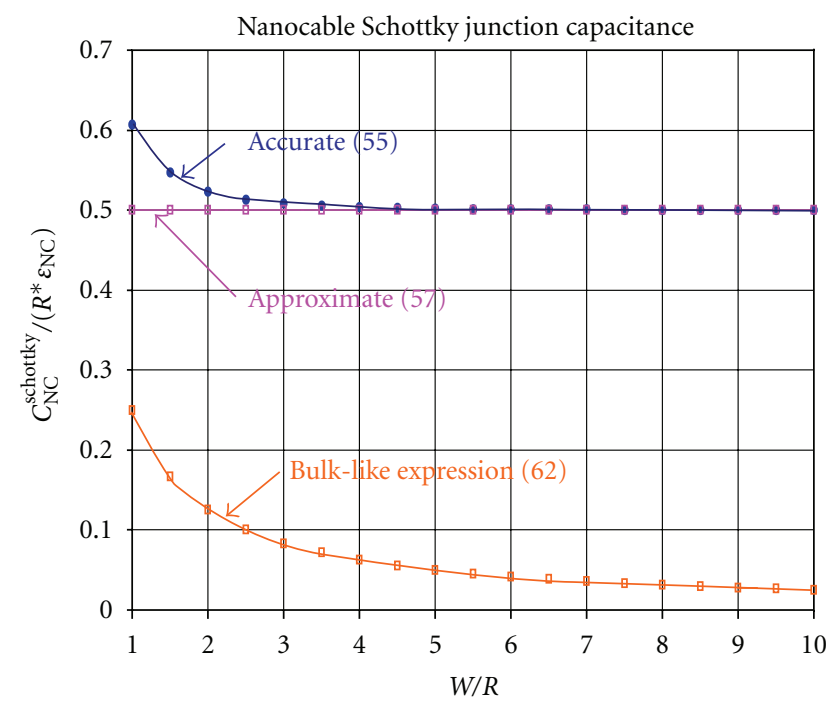

FiguRE 3: Normalized nanocable Schottky junction capacitance against $W / R$. Normalization is to $R \varepsilon_{\mathrm{NC}}$. Formulas (55) and (57) providing $C_{\mathrm{NC}}^{\text {Schottky }}$ are plotted. Also, as a comparison is a result for a bulk-like rendition given by (62).

of the nanocable. Equation (42) must be replaced by

$$
\begin{aligned}
V_{\text {asym, } p}^{\text {ring, cable }}(z) & =\int_{-z_{p}}^{0} K\left(z, z^{\prime}\right) \rho\left(z^{\prime}\right) d z^{\prime} \\
& =-\rho_{p} \frac{2 \pi R t_{\mathrm{NC}}}{\varepsilon_{\mathrm{NC}}} \int_{-z_{p}}^{0} \frac{d z^{\prime}}{\sqrt{R^{2}+\left(z-z^{\prime}\right)^{2}}} \\
& =-\rho_{p} \frac{2 \pi R t_{\mathrm{NC}}}{\varepsilon_{\mathrm{NC}}} \int_{-z_{p}-z}^{-z} \frac{d \bar{z}^{\prime}}{\sqrt{R^{2}+\bar{z}^{\prime 2}}} \\
& =-\left.\rho_{p} \frac{2 \pi R t_{\mathrm{NC}}}{\varepsilon_{\mathrm{NC}}} \ln \left[2\left(\sqrt{R^{2}+\bar{z}^{\prime 2}}+\bar{z}^{\prime}\right)\right]\right|_{-z_{p}-z} ^{-z} \\
& =-\rho_{p} \frac{2 \pi R t_{\mathrm{NC}}}{\varepsilon_{\mathrm{NC}}} \ln \left[\frac{\sqrt{R^{2}+z^{2}}-z}{\sqrt{R^{2}+\left(z_{p}+z\right)^{2}}-z_{p}-z}\right]
\end{aligned}
$$

caused by the charge density depletion separation in the annulus volume

$$
\rho_{\text {asym }}^{\mathrm{NC}}(z)= \begin{cases}\rho_{n}, & 0<z<z_{n} \\ -\rho_{p}, & -z_{p}<z<0, \\ 0, & z<-z_{p} \quad \text { or } \quad z>z_{n} .\end{cases}
$$

Charge neutrality demands that the condition of (41) be generalized for arbitrary depletion widths,

$$
A_{\mathrm{NC}} \int_{0}^{z_{n}} \rho\left(z^{\prime}\right) d z^{\prime}=-A_{\mathrm{NC}} \int_{-z_{p}}^{0} \rho\left(z^{\prime}\right) d z^{\prime}
$$

which because of the unequal but constant doping densities assumed in (68), allows one depletion width to be determined in terms of the other:

$$
z_{p}=\frac{\rho_{n}}{\rho_{p}} z_{n}
$$

Similarly, (39) must be replaced with

$$
\begin{aligned}
& V_{\text {asym, } n}^{\text {ring, cable }}(z) \\
& =\int_{0}^{z_{n}} K\left(z, z^{\prime}\right) \rho\left(z^{\prime}\right) d z^{\prime} \\
& =\rho \frac{2 \pi R t_{\mathrm{NC}}}{\varepsilon_{\mathrm{NC}}} \int_{0}^{z_{n}} \frac{d z^{\prime}}{\sqrt{R^{2}+\left(z-z^{\prime}\right)^{2}}} \\
& =\rho \frac{2 \pi R t_{\mathrm{NC}}}{\varepsilon_{\mathrm{NC}}} \int_{-z}^{z_{n}-z} \frac{d \bar{z}^{\prime}}{\sqrt{R^{2}+\bar{z}^{\prime 2}}} \\
& =\left.\rho \frac{2 \pi R t_{\mathrm{NC}}}{\varepsilon_{\mathrm{NC}}} \ln \left[2\left(\sqrt{R^{2}+\bar{z}^{\prime 2}}+\bar{z}^{\prime}\right)\right]\right|_{-z} ^{z_{n}-z} \\
& =\rho \frac{2 \pi R t_{\mathrm{NC}}}{\varepsilon_{\mathrm{NC}}} \ln \left[\frac{\sqrt{R^{2}+\left(z_{n}-z\right)^{2}}+z_{n}-z}{\sqrt{R^{2}+z^{2}}-z}\right] .
\end{aligned}
$$

The total potential along the nanocable length $z$ will then be a superposition of both the acceptor and donor and charge potentials in (67) and(71)

$$
V_{\text {asym }}^{\mathrm{NC}}=V_{\text {asym, } p}^{\text {ring, cable }}+V_{\text {asym, } n}^{\text {ring, cable }}
$$

or

$$
\begin{aligned}
V_{\mathrm{asym}}^{\mathrm{NC}}(z)= & \frac{2 \pi R t_{\mathrm{NC}}}{\mathcal{E}_{\mathrm{NC}}} \\
& \times\left\{-\rho_{p} \ln \left[\frac{\sqrt{R^{2}+z^{2}}-z}{\sqrt{R^{2}+\left(z_{p}+z\right)^{2}}-z_{p}-z}\right]\right. \\
& \left.+\rho_{n} \ln \left[\frac{\sqrt{R^{2}+\left(z_{n}-z\right)^{2}}+z_{n}-z}{\sqrt{R^{2}+z^{2}}-z}\right]\right\}
\end{aligned}
$$


which makes the junction potential difference

$$
\begin{aligned}
& V_{\text {asym, } j}^{\mathrm{NC}} \\
& =V_{\mathrm{NC}}\left(z_{n}\right)-V_{\mathrm{NC}}\left(-z_{p}\right) \\
& =\frac{2 \pi R t_{\mathrm{NC}}}{\varepsilon_{\mathrm{NC}}} \\
& \times\left\{-\rho_{p} \ln \left[\frac{\sqrt{R^{2}+z_{n}^{2}}-z_{n}}{\sqrt{R^{2}+\left(z_{p}+z_{n}\right)^{2}}-z_{p}-z_{n}}\right]\right. \\
& +\rho_{n} \ln \left[\frac{R}{\sqrt{R^{2}+z_{n}^{2}}-z_{n}}\right]+\rho_{p} \ln \left[\frac{\sqrt{R^{2}+z_{p}^{2}}+z_{p}}{R}\right] \\
& \left.-\rho_{n} \ln \left[\frac{\sqrt{R^{2}+\left(z_{n}+z_{p}\right)^{2}}+z_{n}+z_{p}}{\sqrt{R^{2}+z_{p}^{2}}+z_{p}}\right]\right\} \\
& =\frac{2 \pi R t_{\mathrm{NC}}}{\varepsilon_{\mathrm{NC}}} \\
& \times\left\{-\rho_{p} \ln \left[\frac{\sqrt{R^{2}+z_{n}^{2}}-z_{n}}{\sqrt{R^{2}+z_{p}^{2}}+z_{p}} \frac{R}{\sqrt{R^{2}+\left(z_{p}+z_{n}\right)^{2}}-z_{p}-z_{n}}\right]\right. \\
& \left.+\rho_{n} \ln \left[\frac{R}{\sqrt{R^{2}+\left(z_{n}+z_{p}\right)^{2}}+z_{n}+z_{p}} \frac{\sqrt{R^{2}+z_{p}^{2}}+z_{p}}{\sqrt{R^{2}+z_{n}^{2}}-z_{n}}\right]\right\} .
\end{aligned}
$$

If we define the total depletion width of the nanocable as

$$
W_{\mathrm{asym}}^{\mathrm{NC}}=z_{p}+z_{n}=\left(\frac{\rho_{n}}{\rho_{p}}+1\right) z_{n}
$$

then when we examine the case when the $p$-region doping density gets large, $\rho_{p} \rightarrow \infty,(70)$ and (75) will reduce $V_{\mathrm{asym}, j}^{\mathrm{NC}}$ to the form of (49). That is, a Schottky junction consisting of a perfect infinite metal plane contacting an $n$-doped nanocable is equivalent to an asymmetric $p$ - $n$ junction when the $p$-doping becomes extremely large compared to the $n$ doping.

For the situation of a symmetric junction, when $\rho_{p}=$ $\rho_{n}=\rho$, and (70) becomes

$$
z_{p}=\frac{\rho_{n}}{\rho_{p}} z_{n}=z_{n}=W
$$

and (74) reduces to

$$
\begin{aligned}
& V_{\text {sym, } j}^{\mathrm{NC}}=V_{\mathrm{NC}}(W)- V_{\mathrm{NC}}(-W) \\
&=\rho \frac{2 \pi R t_{\mathrm{NC}}}{\varepsilon_{\mathrm{NC}}}\left\{2 \ln \left[\frac{\sqrt{R^{2}+W^{2}}+W}{\sqrt{R^{2}+W^{2}}-W}\right]\right. \\
&\left.+\ln \left[\frac{\sqrt{R^{2}+4 W^{2}}-2 W}{\sqrt{R^{2}+4 W^{2}}+2 W}\right]\right\} .
\end{aligned}
$$

(One notes that the symmetry properties $V_{\text {sym, } n}^{\mathrm{NC}}(W)=$ $-V_{\text {sym, } p}^{\mathrm{NC}}(-W)$ and $V_{\text {sym, } n}^{\mathrm{NC}}(-W)=-V_{\text {sym }, p}^{\mathrm{NC}}(W)$ are satisfied by (67) and (71), and cause $V_{\text {sym, } j}^{\mathrm{NC}}=2 V_{\text {sym }}^{\mathrm{NC}}(W)$.) Consider the limit of expression (77) when $W / R \rightarrow \infty$. The nanocable junction voltage reduces to

$$
\left.V_{\mathrm{sym}, j}^{\mathrm{NC}}\right|_{W / R \gg 1} \approx \rho \frac{4 \pi R t_{\mathrm{NC}}}{\varepsilon_{\mathrm{NW}}} \ln \left(\frac{W}{R}\right)
$$

which is a very different form of junction voltage dependence on $W$ than that for the Schottky nanocable junction seen in (51). It has gone from a linear to a logarithmic dependence. Using a formula like in (46) for the capacitance, namely,

$$
C_{\mathrm{NC}}^{\mathrm{sym}}= \pm \frac{d Q_{\mathrm{NC}}^{\mathrm{sym}}}{d V_{\mathrm{bias}}}= \pm e N_{d} A_{\mathrm{NC}} \frac{d W\left(V_{\text {bias }}\right)}{d V_{\text {bias }}}
$$

we see that solving for $W$ in(78)

$$
W\left(V_{j}\right)=\operatorname{Re}^{\varepsilon_{\mathrm{NC}} V_{j} /\left(4 \pi \rho R t_{\mathrm{NC}}\right)}
$$

and taking the derivative, yields

$$
C_{\mathrm{NC}}^{\mathrm{sym}}=\frac{1}{2} \varepsilon_{\mathrm{NC}} W .
$$

The exponent $\gamma$ in (80), for low bias voltages having $V_{j}=$ $V_{\mathrm{bi}}$, and for the values used for the $\mathrm{RuO}_{2} / \mathrm{SiO}_{2}$ nanocables before, namely, $V_{\mathrm{bi}}=0.42 \mathrm{~V}$ and $R \rightarrow R_{a v}^{\text {cable }}=31 \mathrm{~nm}$ and $t_{\mathrm{NC}} \rightarrow \Delta R^{\text {cable }}=2 \mathrm{~nm}$, with $N_{\mathrm{NC}}=N_{d}=10^{19} / \mathrm{cc}\left(\rho=e N_{d}\right)$, $\gamma=0.13905$ making $W=1.149 \mathrm{R}$. Clearly, this does not satisfy $W \gg R$ well. But if $N_{d}$ was an order of magnitude smaller, then $W=4.0169 \mathrm{R}$, which is respectable. For the larger doping value, we calculate

$$
C_{\mathrm{NC}, \mathrm{sym}}^{\mathrm{RuO}_{2} / \mathrm{SiO}_{2}}=6.95 \mathrm{aF}, \quad N_{d}=1.0 \times 10^{19} / \mathrm{cc}, \quad \varepsilon_{\mathrm{NC}}=3.69 .
$$

Capacitance can be determined exactly by taking the derivative of (77), finding

$$
C_{\mathrm{NC}}^{\mathrm{sym}}=\frac{\varepsilon_{\mathrm{NC}}}{2} \frac{\sqrt{R^{2}+W^{2}}}{2-\sqrt{\left(R^{2}+W^{2}\right) /\left(R^{2}+4 W^{2}\right)}} .
$$

To obtain $C_{\mathrm{NC}}^{\text {sym }}$ in terms of $V_{\mathrm{bi}}$, set $V_{j}^{\mathrm{NC}}=V_{\mathrm{bi}}$ on the lefthand side of (77):

$$
\begin{aligned}
& V_{\mathrm{bi}}=\rho \frac{2 \pi R t_{\mathrm{NC}}}{\varepsilon_{\mathrm{NC}}}\{2 \ln \left[\frac{\sqrt{R^{2}+W^{2}}+W}{\sqrt{R^{2}+W^{2}}-W}\right] \\
&\left.+\ln \left[\frac{\sqrt{R^{2}+4 W^{2}}-2 W}{\sqrt{R^{2}+4 W^{2}}+2 W}\right]\right\}
\end{aligned}
$$

and solve for $W\left(V_{\mathrm{bi}}\right)$, and insert this into (83). One might 
wonder what form is obtained for $C_{\mathrm{NC}}^{\text {sym }}$ by taking the limit $W / R \rightarrow \infty$ in (83) after its formula has been derived:

$$
C_{\mathrm{NC}}^{\mathrm{sym}}=\frac{1}{3} \varepsilon_{\mathrm{NC}} W
$$

which differs slightly from (81), making the capacitance somewhat smaller,

$$
C_{\mathrm{NC}, \mathrm{sym}}^{\mathrm{RuO}_{2} / \mathrm{SiO}_{2}}=4.64 \mathrm{aF}, \quad N_{d}=1.0 \times 10^{19} / \mathrm{cc}, \quad \varepsilon_{\mathrm{NC}}=3.69 .
$$

Comparison of the most general formula (83) for capacitance of a symmetric nanocable junction $C_{\mathrm{NC}}^{\mathrm{sym}}$ with either (81) or (85) in Figure 4, shows that the less accurate formulas seem to bracket it, with (81) almost always being greater than it, whereas (85) is always slightly less. Equation (81) diverges from (83) noticeably as the $W / R$ ratio increases.

Table 1 summarizes the nanocable capacitance formulas found in the last subsection and in this subsection. The formulas are given in unitless form because each capacitance is normalized to $R \varepsilon_{i}$ (this product's units is Farads) where $i=\mathrm{NC}$, NW. That is, the capacitance is provided as $C /\left(R \varepsilon_{i}\right)$.

\section{Semiconductor p-n Junction Capacitances of Nanowires}

The symmetric $p-n$ junction for semiconductor nanowires is a basic building block of nanowire devices, and would be of great interest to determine its capacitance. The nanocable potential functions cannot be used because they only include a thin annulus of semiconducting cross-section, while the nanowire has a disk cross-section.

In the next subsection, we will first look at the high symmetry case of equal doping on either side of the semiconductor nanowire junction. After that, the much more complicated case of an abrupt asymmetric $p-n$ semiconductor nanowire junction will be addressed in the second subsection. Here the junction potential difference as a function of its $p$ - and $n$-type depletion widths will be found, and the limiting case of an infinitely high $p$-type doping density will be studied. That Schottky-like capacitance will be determined.

7.1. Symmetric Semiconductor $p-n$ Junction Capacitance of Nanowires. We will look at the symmetric semiconductor nanowire $p-n$ junction here (Figure 5(a)). First specify the depletion region charge density, which in the abrupt approximation, changes from (40) to

$$
\rho_{\mathrm{sym}}^{\mathrm{NW}}(z)= \begin{cases}\rho, & 0<z<W \\ -\rho, & -W<z<0, \\ 0, & |z|>W .\end{cases}
$$

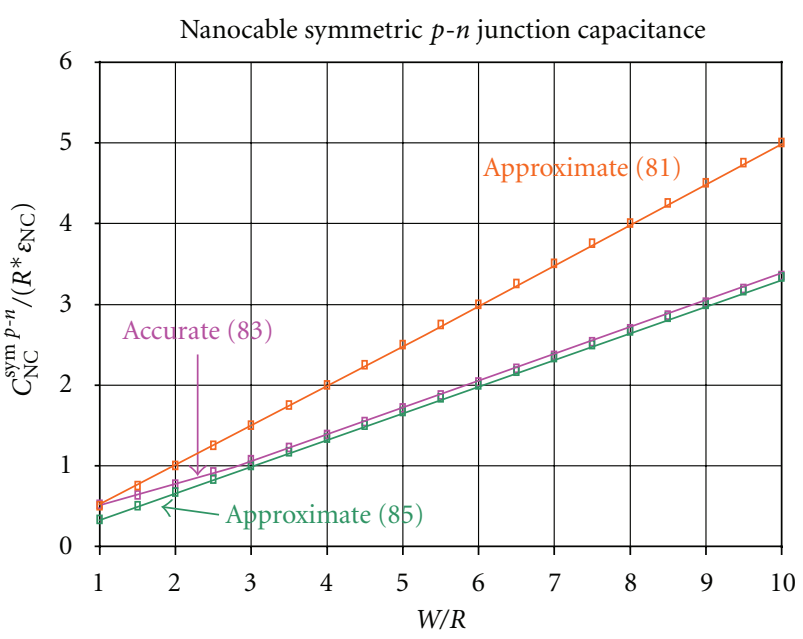

Figure 4: Normalized nanocable symmetric $p$ - $n$ semiconductor junction capacitance against $W / R$. Normalization is to $R \varepsilon_{\mathrm{NC}}$. Formulas (81), (83), and (85) providing $\mathrm{C}_{\mathrm{NC}}^{\text {sym }}$ are plotted.

By inspecting the integral formula for a vacuum potential solution [62], the on axis value for the nanowire is (use transformation $\left.u=s^{2}+\left(z-z^{\prime}\right)^{2}, d u=2 s d s\right)$

$$
\begin{aligned}
\varphi_{\mathrm{NW}} & (0,0, z) \\
= & \frac{1}{\varepsilon_{\mathrm{NW}}} \int_{-\infty}^{\infty} d z^{\prime} \int_{0}^{R} s d s \int_{0}^{2 \pi} d \phi \frac{\rho\left(s, \phi, z^{\prime}\right)}{\sqrt{s^{2}+\left(z-z^{\prime}\right)^{2}}} \\
= & \frac{2 \pi}{\varepsilon_{\mathrm{NW}}} \int_{-\infty}^{\infty} d z^{\prime} \int_{0}^{R} s d s \frac{\rho\left(s, z^{\prime}\right)}{\sqrt{s^{2}+\left(z-z^{\prime}\right)^{2}}} \\
= & \frac{2 \pi}{\varepsilon_{\mathrm{NW}}} \int_{-\infty}^{\infty} \rho\left(z^{\prime}\right) d z^{\prime} \int_{0}^{R} \frac{s d s}{\sqrt{s^{2}+\left(z-z^{\prime}\right)^{2}}} \\
= & \frac{2 \pi}{\varepsilon_{\mathrm{NW}}} \int_{-\infty}^{\infty} \rho\left(z^{\prime}\right)\left[\sqrt{R^{2}+\left(z-z^{\prime}\right)^{2}}-\left(z-z^{\prime}\right)\right] d z^{\prime}
\end{aligned}
$$

leading to the kernel $(||=\operatorname{abs}()$ operator is chosen leading to the correct branch cuts)

$$
K\left(z, z^{\prime}\right)=\frac{2 \pi}{\varepsilon_{\mathrm{NW}}}\left[\sqrt{R^{2}+\left(z-z^{\prime}\right)^{2}}-\left|z-z^{\prime}\right|\right]
$$

which is used for calculating the potential from the $p$-side of the junction [76], see [76, page 59, 260.01 and page 60 , 262.01 ], and also [64, page $86,2.271,3$, with $I_{1}$ for $c>0$ ]

$$
\begin{aligned}
V_{\text {sym, } p}^{\mathrm{NW}}(z) & =\int_{-W}^{0} K\left(z, z^{\prime}\right) \rho\left(z^{\prime}\right) d z^{\prime} \\
& =\frac{2 \pi}{\varepsilon_{\mathrm{NW}}} \int_{-W}^{0} \rho\left(z^{\prime}\right)\left[\sqrt{R^{2}+\left(z-z^{\prime}\right)^{2}}+\left(z-z^{\prime}\right)\right] d z^{\prime} \\
& =-\rho \frac{2 \pi}{\varepsilon_{\mathrm{NW}}}\left\{\int_{X_{-W}}^{X_{0}} \frac{X^{2} d X}{\sqrt{X^{2}-R^{2}}}+z W\left(1+\frac{1}{2} \frac{W}{z}\right)\right\}
\end{aligned}
$$




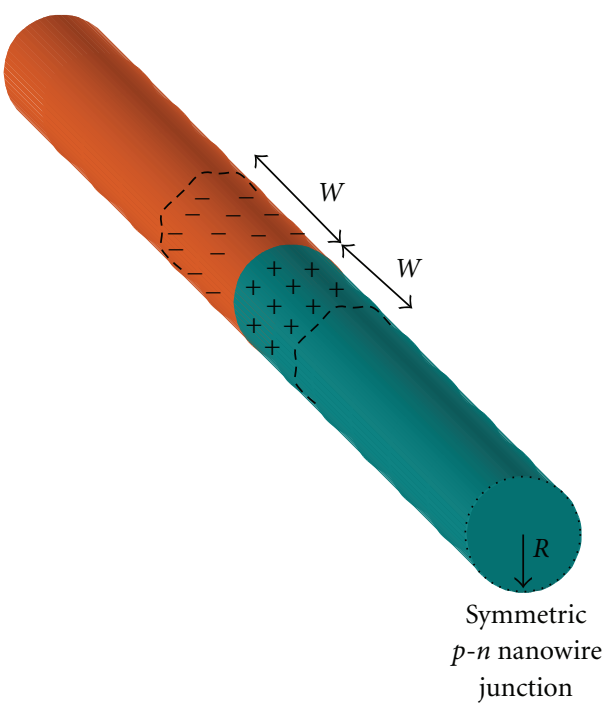

(a)

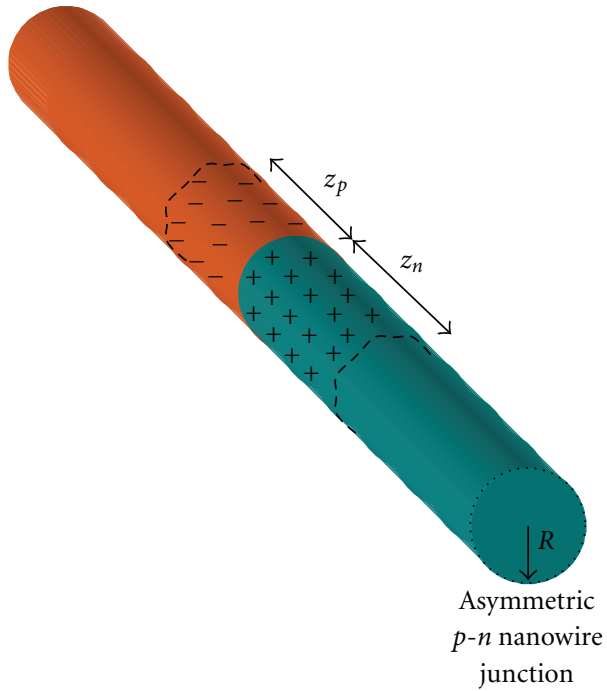

(b)

Figure 5: (a) Symmetric semiconductor $p-n$ nanowire junction. (b) Asymmetric semiconductor $p$ - $n$ nanowire junction. This is examined for the limiting case when $\rho_{p} \rightarrow \infty$.

$$
\begin{aligned}
= & -\rho \frac{2 \pi}{\varepsilon_{\mathrm{NW}}} \\
& \times\left\{\frac{(z+W) \sqrt{R^{2}+(z+W)^{2}}-z \sqrt{R^{2}+z^{2}}}{2}\right. \\
& -\frac{R^{2}}{2} \ln \left[\frac{\sqrt{R^{2}+(z+W)^{2}}-(z+W)}{\sqrt{R^{2}+z^{2}}-z}\right] \\
& \left.\mp z W\left(1+\frac{1}{2} \frac{W}{z}\right)\right\} .
\end{aligned}
$$

$$
\begin{aligned}
& =\rho \frac{2 \pi}{\varepsilon_{\mathrm{NW}}} \\
& \quad \times\left\{\frac{(W-z) \sqrt{R^{2}+(z-W)^{2}}+z \sqrt{R^{2}+z^{2}}}{2}\right. \\
& \\
& \quad-\frac{R^{2}}{2} \ln \left[\frac{\sqrt{R^{2}+(z-W)^{2}}+z-W}{\sqrt{R^{2}+z^{2}}+z}\right] \\
& \left.\quad \mp z W\left(1-\frac{1}{2} \frac{W}{z}\right)\right\} .
\end{aligned}
$$

where the change of variables $X^{2}=R^{2}+\left(z-z^{\prime}\right)^{2},-(z-$ $\left.z^{\prime}\right) d z^{\prime}=X d X$ was used. (Note, care is required in selecting correct branch cuts, and we use $-\sqrt{X^{2}-R^{2}}$ in the third line of (90), and $X=-\sqrt{R^{2}+\left(z-z^{\prime}\right)^{2}}$ and $S=+\sqrt{X^{2}-R^{2}}$ in the fourth line of (90).) The potential from the $n$-side of the junction will be

$$
\begin{aligned}
& V_{\text {sym, } n}^{\mathrm{NW}}(z) \\
& \quad=\int_{0}^{W} K\left(z, z^{\prime}\right) \rho\left(z^{\prime}\right) d z^{\prime} \\
& \quad=\rho \frac{2 \pi}{\varepsilon_{\mathrm{NW}}} \int_{0}^{W}\left[\sqrt{R^{2}+\left(z-z^{\prime}\right)^{2}}-\left(z-z^{\prime}\right)\right] d z^{\prime} \\
& \quad=\rho \frac{2 \pi}{\varepsilon_{\mathrm{NW}}}\left\{\left.\left[\frac{X S}{2}+\frac{R^{2}}{2} \ln |X+S|\right]\right|_{X_{0}} ^{X_{W}}-z W\left(1-\frac{1}{2} \frac{W}{z}\right)\right\}
\end{aligned}
$$

In (90) and (91), the $\mp$ symbol has its “-” and "+" signs refer to, respectively, $z \geq W$ and $z \leq-W$. Thus the electrostatic Green's function for the bounded or partitioned problem in $z$-space is specified in two out of its three spatial regions. Since we will not be making evaluations in the interior depletion charged region $-W<z<W$, it is not supplied here, although it can also be determined. Branch cuts selected for $V_{\text {sym, } p}^{\mathrm{NW}}(z)$ and $V_{\text {sym, } n}^{\mathrm{NW}}(z)$ satisfy the physical symmetry and limiting conditions

$$
\begin{gathered}
V_{\text {sym, } n}^{\mathrm{NW}}(W)=-V_{\text {sym, } p}^{\mathrm{NW}}(-W), \\
V_{\text {sym, } n}^{\mathrm{NW}}(-W)=-V_{\text {sym, } p}^{\mathrm{NW}}(W), \\
\lim _{z \rightarrow \infty} V_{\text {sym, } n}^{\mathrm{NW}}(z)=-\lim _{z \rightarrow-\infty} V_{\text {sym, } p}^{\mathrm{NW}}(z)=\rho \pi R^{2} W / z .
\end{gathered}
$$

The total potential due to both the $p$ - and $n$-sides of the junction depletion region will be (mobile carriers are neglected, which would migrate to the outer part of 
the nanowire cylinder, and not allow $\rho\left(z^{\prime}\right)=\rho=$ const to be extracted from the integration), enlisting (90) and (91),

$$
V_{\text {sym }}^{\mathrm{NW}}(z)=V_{\text {sym, } p}^{\mathrm{NW}}(z)+V_{\text {sym, } n}^{\mathrm{NW}}(z)
$$

or

$$
\begin{aligned}
& V_{\text {sym }}^{\mathrm{NW}}(z) \\
& =-\rho \frac{2 \pi}{\varepsilon_{\mathrm{NW}}}\left\{\frac { 1 } { 2 } \left[(z+W) \sqrt{R^{2}+(z+W)^{2}}\right.\right. \\
& \left.-(W-z) \sqrt{R^{2}+(z-W)^{2}}\right] \\
& -z \sqrt{R^{2}+z^{2}}-\frac{1}{2} R^{2} \\
& \quad \times \ln \left[\frac{\sqrt{R^{2}+(z+W)^{2}}-(z+W)}{\sqrt{R^{2}+(z-W)^{2}}+z-W} \frac{\sqrt{R^{2}+z^{2}}+z}{\sqrt{R^{2}+z^{2}}-z}\right] \\
& \\
& \left.\mp W^{2}\right\} .
\end{aligned}
$$

Junction voltage is found from a relationship like in (72), using either the general formula (95), or more simply, from the symmetry conditions in (92), which make

$$
\begin{aligned}
V_{\mathrm{sym}, j}^{\mathrm{NW}}= & V_{\mathrm{NW}}(W)-V_{\mathrm{NW}}(-W) \\
= & 2\left[V_{p}^{\mathrm{NW}}(W)+V_{n}^{\mathrm{NW}}(W)\right] \\
= & 2 V_{\mathrm{sym}}^{\mathrm{NW}}(W) . \\
V_{\mathrm{sym}, j}^{\mathrm{NW}}= & \rho \frac{4 \pi}{\varepsilon_{\mathrm{NW}}}\left\{W \sqrt{R^{2}+W^{2}}-W \sqrt{R^{2}+(2 W)^{2}}+\frac{R^{2}}{2}\right. \\
& \quad \times \ln \left[\frac{\sqrt{(2 W)^{2}+R^{2}}-2 W}{R} \frac{\sqrt{W^{2}+R^{2}}+W}{\sqrt{W^{2}+R^{2}}-W}\right] \\
& \left.\quad+W^{2}\right\} .
\end{aligned}
$$

Again it should be noted that a more accurate form of $C_{\mathrm{NW}}$ can be found by directly using formula in (97) and taking the derivative of both sides of that equation with respect to $V_{\text {bias }}$, utilizing (48), solving for $d W / d V_{\text {bias }}$, and inserting that into a capacitive expression like that of (55). We will not do that here first, but obtain a simpler form instead by considering the limiting form for $W / R \rightarrow \infty$, which allows

$$
\left.V_{\mathrm{sym}, j}^{\mathrm{NW}}\right|_{W / R \gg 1} \approx \rho \frac{\pi R^{2}}{\varepsilon_{\mathrm{NW}}}\left[1+2\left(\frac{R}{W}\right)^{2} \ln \frac{W}{R}\right] .
$$

Equation (95) does not allow $W$ to be expressed as, say $\left.W_{\mathrm{NW}}\right|_{W / R \gg 1}=\sqrt{\left.\varepsilon_{\mathrm{NW}} V_{\text {sym, } j}^{\mathrm{NW}}\right|_{W / R \gg 1} /\left(4 \pi e N_{d}\right)}$, which for an unbiased device, replacing the junction voltage by the builtin voltage, gives $\left.W_{\mathrm{bi}}^{\mathrm{NW}}\right|_{W / R \gg 1}=\sqrt{\varepsilon_{\mathrm{NW}} V_{\mathrm{bi}} /\left(4 \pi e N_{d}\right)}$, whose form is reminiscent of the two-sided abrupt junction depletion width seen in [66], namely, $W=\operatorname{sqrt}\left(4 \varepsilon_{s} V_{\mathrm{bi}} /\left[q N_{B}\right]\right)$ (Factor of $1 /[16 \pi]$ under the square root operator, has $1 /[4 \pi]$ of it due to the use of cgs units.)

The capacitance of the $p-n$ nanowire junction with area $A_{\mathrm{NW}}=\pi R^{2}$, will be using (98),

$$
\begin{aligned}
C_{\mathrm{NW}}^{p-n} & = \pm \frac{d Q_{\mathrm{NW}}}{d V_{\text {bias }}}= \pm \pi R^{2} \rho \frac{d W\left(V_{\text {bias }}\right)}{d V_{\text {bias }}} \\
& =\frac{1}{2} \varepsilon_{\mathrm{NW}} W \frac{(W / R)^{2}}{1-2 \ln (W / R)} .
\end{aligned}
$$

Formula (99) is really meant to be used for large $W / R$ ratios, but often the trend can be found for modest $W / R$ values. However, we see here that a singularity occurs at $W / R=$ $e^{1 / 2} \approx 1.65$ and that even if we kept the $\ln (W / R)$ factor in the denominator, the expression would be useless at low $W / R$ values near one.

To obtain a more accurate form of $C_{\mathrm{NW}}^{p-n}$, employ (97) and take its derivative on both sides of that equation with respect to $V_{\text {bias }}$, utilizing (48), solving for $d W / d V_{\text {bias }}$, and inserting that into a capacitive expression like (79), namely, the first line of(99)

$$
\begin{aligned}
C_{\mathrm{NW}}^{p-n}=\frac{R \varepsilon_{\mathrm{NW}}}{4}[ & \sqrt{1+\left(\frac{W}{R}\right)^{2}}-\sqrt{1+4\left(\frac{W}{R}\right)^{2}} \\
& +\frac{W / R}{\sqrt{1+(R / W)^{2}}}-\frac{2 W / R}{\sqrt{1+(R / 2 W)^{2}}} \\
& \left.+\frac{1}{\sqrt{1+(W / R)^{2}}}-\frac{1}{\sqrt{1+(2 W / R)^{2}}}+\frac{2 W}{R}\right]^{-1} .
\end{aligned}
$$

To obtain $C_{\mathrm{NW}}^{p-n}$ in terms of $V_{\mathrm{bi}}$, set $V_{j}^{\mathrm{NW}}=V_{\mathrm{bi}}$ on the lefthand side of (97):

$$
\begin{aligned}
V_{\mathrm{bi}}= & \rho \frac{4 \pi}{\varepsilon_{\mathrm{NW}}} \\
& \times\left\{W \sqrt{R^{2}+W^{2}}-W \sqrt{R^{2}+4 W^{2}}\right. \\
& \quad+\frac{R^{2}}{2} \ln \left[\frac{\sqrt{4 W^{2}+R^{2}}-2 W}{R} \frac{\sqrt{W^{2}+R^{2}}+W}{\sqrt{W^{2}+R^{2}}-W}\right] \\
& \left.+W^{2}\right\}
\end{aligned}
$$

and solve for $W\left(V_{\mathrm{bi}}\right)$, and insert this into (100).

An approximation to the nanowire $p-n$ junction capacitance capacitance may be examined for large $W / R$ by 
taking the limit of (100) for $W / R \rightarrow \infty$, with the result

$$
C_{\mathrm{NW}}^{p-n}=\varepsilon_{\mathrm{NW}} W
$$

Evaluating (102) for a small radius nanowire like the previously examined carbon nanotube, with $R=1 \mathrm{~nm}$, gives

$$
C_{\mathrm{NW}}^{p-n}=0.113 \mathrm{aF}, \quad \varepsilon_{\mathrm{NW}}=1
$$

Alternatively, evaluating (102) for a large radius nanowire like the previously examined $\mathrm{RuO}_{2} / \mathrm{SiO}_{2}$ nanocable, with $R=31 \mathrm{~nm}$, gives

$$
C_{\mathrm{NW}}^{p-n}=12.7 \mathrm{aF}, \quad \varepsilon_{\mathrm{NW}}=3.69 .
$$

Figure 6 shows the dependence of nanowire junction capacitance $C_{\mathrm{NW}}^{p-n}$ on $W / R$. The approximate formula (102) and the more accurate formula (100) increasingly diverge from each other as $W / R$ increases, with the approximate relation overestimating capacitance in excess of a factor of two at $W / R=10$.

7.2. Asymmetric Semiconductor $p-n$ Junction Capacitance of Nanowires. The asymmetric nanowire semiconductor $p-n$ junction (Figure 3(b)) is considerably more involved than the previous symmetric nanowire case. Depletion region charge density of the nanowire is generalized from (87), as was the nanocable in (66), to

$$
\rho_{\text {asym }}^{\mathrm{NW}}(z)= \begin{cases}\rho_{n}, & 0<z<z_{n} \\ -\rho_{p}, & -z_{p}<z<0, \\ 0, & z<-z_{p} \quad \text { or } \quad z>z_{n} .\end{cases}
$$

Potential contribution from the $p$-side of the nanowire junction, using (105), will be

$$
\begin{aligned}
& V_{\text {asym, } p}^{\mathrm{NW}}(z) \\
& =\int_{-z_{p}}^{0} K\left(z, z^{\prime}\right) \rho\left(z^{\prime}\right) d z^{\prime} \\
& =\frac{2 \pi}{\varepsilon_{\mathrm{NW}}} \int_{-z_{p}}^{0} \rho\left(z^{\prime}\right)\left[\sqrt{R^{2}+\left(z-z^{\prime}\right)^{2}}+\left(z-z^{\prime}\right)\right] d z^{\prime}
\end{aligned}
$$

$$
\begin{aligned}
& =-\rho_{p} \frac{2 \pi}{\varepsilon_{\mathrm{NW}}}\left\{\int_{X_{-z_{p}}}^{X_{0}} \frac{X^{2} d X}{\sqrt{X^{2}-R^{2}}}+z z_{p}\left(1+\frac{1}{2} \frac{z_{p}}{z}\right)\right\} \\
& =-\rho_{p} \frac{2 \pi}{\varepsilon_{\mathrm{NW}}}\left\{\frac{\left(z+z_{p}\right) \sqrt{R^{2}+\left(z+z_{p}\right)^{2}}-z \sqrt{R^{2}+z^{2}}}{2}\right.
\end{aligned}
$$$$
-\frac{R^{2}}{2} \ln \left[\frac{\sqrt{R^{2}+\left(z+z_{p}\right)^{2}}-\left(z+z_{p}\right)}{\sqrt{R^{2}+z^{2}}-z}\right]
$$$$
\left.\mp z z_{p}\left(1+\frac{1}{2} \frac{z_{p}}{z}\right)\right\} .
$$

Likewise, the potential contribution from the n-side of the nanowire junction will be

$$
\begin{aligned}
& V_{\mathrm{asym}, n}^{\mathrm{NW}}(z)=\int_{0}^{z_{n}} K\left(z, z^{\prime}\right) \rho\left(z^{\prime}\right) d z^{\prime} \\
& =\rho_{n} \frac{2 \pi}{\varepsilon_{\mathrm{NW}}} \int_{0}^{z_{n}}\left[\sqrt{R^{2}+\left(z-z^{\prime}\right)^{2}}-\left(z-z^{\prime}\right)\right] d z^{\prime} \\
& =\rho_{n} \frac{2 \pi}{\varepsilon_{\mathrm{NW}}}\left\{\left.\left[\frac{X S}{2}+\frac{R^{2}}{2} \ln |X+S|\right]\right|_{X_{0}} ^{X_{z_{n}}}\right. \\
& \left.-z z_{n}\left(1-\frac{1}{2} \frac{z_{n}}{z}\right)\right\} \\
& =\rho_{n} \frac{2 \pi}{\varepsilon_{\mathrm{NW}}} \\
& \times\left\{\frac{\left(z_{n}-z\right) \sqrt{R^{2}+\left(z-z_{n}\right)^{2}}+z \sqrt{R^{2}+z^{2}}}{2}\right. \\
& -\frac{R^{2}}{2} \ln \left[\frac{\sqrt{R^{2}+\left(z-z_{n}\right)^{2}}+z-z_{n}}{\sqrt{R^{2}+z^{2}}+z}\right] \\
& \left.\mp z z_{n}\left(1-\frac{1}{2} \frac{z_{n}}{z}\right)\right\} .
\end{aligned}
$$

Total potential due to both the $p$ - and $n$-sides of the asymmetric nanowire junction depletion region will be

$$
V_{\text {asym }}^{\mathrm{NW}}(z)=V_{\text {asym, } p}^{\mathrm{NW}}(z)+V_{\text {asym, } n}^{\mathrm{NW}}(z)
$$


and inserting into this (106) and (107) yields

$$
\begin{aligned}
& V_{\mathrm{asym}}^{\mathrm{NW}}(z) \\
& =\frac{2 \pi}{\varepsilon_{\mathrm{NW}}}\left\{\rho_{p} \frac{z \sqrt{R^{2}+z^{2}}-\left(z+z_{p}\right) \sqrt{R^{2}+\left(z+z_{p}\right)^{2}}}{2}\right. \\
& +\rho_{p} \frac{R^{2}}{2} \ln \left[\frac{\sqrt{R^{2}+\left(z+z_{p}\right)^{2}}-\left(z+z_{p}\right)}{\sqrt{R^{2}+z^{2}}-z}\right] \\
& \quad \pm \rho_{p} z z_{p}\left(1+\frac{\left.1 \frac{z_{p}}{2}\right)}{z}\right] \\
& +\rho_{n} \frac{\left(z_{n}-z\right) \sqrt{R^{2}+\left(z-z_{n}\right)^{2}}+z \sqrt{R^{2}+z^{2}}}{2} \\
& \quad-\rho_{n} \frac{R^{2}}{2} \ln \left[\frac{\sqrt{R^{2}+\left(z-z_{n}\right)^{2}}+z-z_{n}}{\sqrt{R^{2}+z^{2}}+z}\right] \\
& \left.\mp \rho_{n} z z_{n}\left(1-\frac{1}{2} \frac{z_{n}}{z}\right)\right\} . \\
&
\end{aligned}
$$

Junction voltage is calculated from

$$
V_{\mathrm{asym}, j}^{\mathrm{NW}}=\mathrm{V}_{\mathrm{asym}}^{\mathrm{NW}}\left(z_{n}\right)-V_{\mathrm{asym}}^{\mathrm{NW}}\left(-z_{p}\right)
$$

and when evaluating (109) at $z=z_{n},-z_{p}$, with a total nanowire depletion width $W_{T}=W_{\text {asym }}^{\mathrm{NW}}=z_{p}+z_{n}$ we find

$$
\begin{aligned}
& V_{\mathrm{asym}, j}^{\mathrm{NW}} \frac{2 \pi}{\varepsilon_{\mathrm{NW}}}\left\{-\frac{W_{T} \sqrt{R^{2}+W_{T}^{2}}}{2}\left(\rho_{p}+\rho_{n}\right)\right. \\
& +\frac{z_{n} \rho_{p} \sqrt{R^{2}+z_{n}^{2}}+z_{p} \rho_{n} \sqrt{R^{2}+z_{p}^{2}}}{2} \\
& +\frac{R^{2}}{2}\left\{\rho_{p} \ln \left[\frac{\sqrt{R^{2}+W_{T}^{2}}-W_{T}}{\sqrt{R^{2}+z_{n}^{2}}-z_{n}}\right]\right. \\
& \left.+\rho_{n} \ln \left[\frac{\sqrt{R^{2}+W_{T}^{2}}-W_{T}}{\sqrt{R^{2}+z_{p}^{2}}-z_{p}}\right]\right\}
\end{aligned}
$$

$$
\begin{aligned}
& +z_{n} z_{p}\left(\rho_{p}+\rho_{n}\right) \\
& -\frac{z_{p} \rho_{p} \sqrt{R^{2}+z_{p}^{2}}-z_{n} \rho_{n} \sqrt{R^{2}+z_{n}^{2}}}{2} \\
& -\frac{R^{2}}{2}\left\{\rho_{n} \ln \left[\frac{R}{\sqrt{R^{2}+z_{n}^{2}}+z_{n}}\right]\right. \\
& \left.+\rho_{p} \ln \left[\frac{R}{\sqrt{R^{2}+z_{p}^{2}}+z_{p}}\right]\right\}
\end{aligned}
$$

Now consider the case when the $p$-side becomes highly doped, $\rho_{p} \rightarrow \infty$. Formula (111) becomes, if one also allows $z_{n} \gg R$ (implies $W_{T}>z_{n} \gg R$ ),

$$
V_{\mathrm{asym}}^{\mathrm{NW}} \mid \underset{\substack{\rho_{p} \rightarrow \infty \\ W / R \gg 1}}{ }=\rho_{n} \frac{\pi W^{2}}{\varepsilon_{\mathrm{NW}}}
$$

when simplifying notation $W_{T} \rightarrow z_{n}=W$. Because this is similar to the altered form of (51) when we studied the effect then of a quadratic $W$ dependence, we may write $W$ as

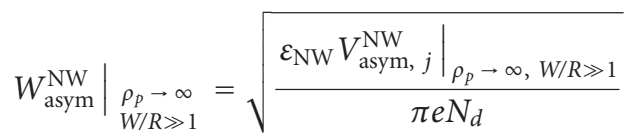

and giving for an unbiased device,

$$
\left.\mathrm{W}_{\mathrm{bi}}^{\mathrm{NW}}\right|_{\substack{\rho_{p \rightarrow \infty} \rightarrow R \gg 1 \\ W / R \gg 1}}=\sqrt{\frac{\varepsilon_{\mathrm{NW}} V_{\mathrm{bi}}}{\pi e N_{d}}} .
$$

The capacitance will be, enlisting (113),

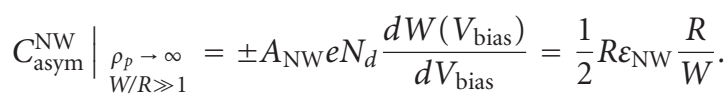

In mks units this would yield $\left.C_{\text {asym }}^{\mathrm{NW}}\right|_{\rho_{p} \rightarrow \infty, W / R \gg 1}=$ $2 \pi R^{2} \varepsilon_{\mathrm{NW}} / W$, and the per-unit area capacitance would be $\left.C_{\mathrm{asym}}^{\mathrm{NW}, u a}\right|_{\rho_{p} \rightarrow \infty, W / R \gg 1}=2 \varepsilon_{\mathrm{NW}} / W$, displaying the familiar form [62] which is inversely proportional to $W$. Evaluating (115) for a large radius nanowire like the previously examined $\mathrm{RuO}_{2} / \mathrm{SiO}_{2}$ nanocable, with $R=31 \mathrm{~nm}$, gives

$$
\begin{array}{c|c}
C_{\mathrm{asym}}^{\mathrm{NW}} \mid \underset{W / R \gg 1}{\rho_{p} \rightarrow \infty}=23.189 \mathrm{aF}, \\
N_{d}=5 \times 10^{18} / \mathrm{cc}, \quad \varepsilon_{\mathrm{NW}}=3.69 .
\end{array}
$$

However, if evaluated for a $R=1 \mathrm{~nm}$ radius,

$$
\begin{gathered}
\mathrm{C}_{\mathrm{asym}}^{\mathrm{NW}} \mid \underset{W / R \gg 1}{\rho_{p} \rightarrow \infty}=0.0241 \mathrm{aF}, \\
N_{d}=5 \times 10^{18} / \mathrm{cc}, \quad \varepsilon_{\mathrm{NW}}=3.69 .
\end{gathered}
$$




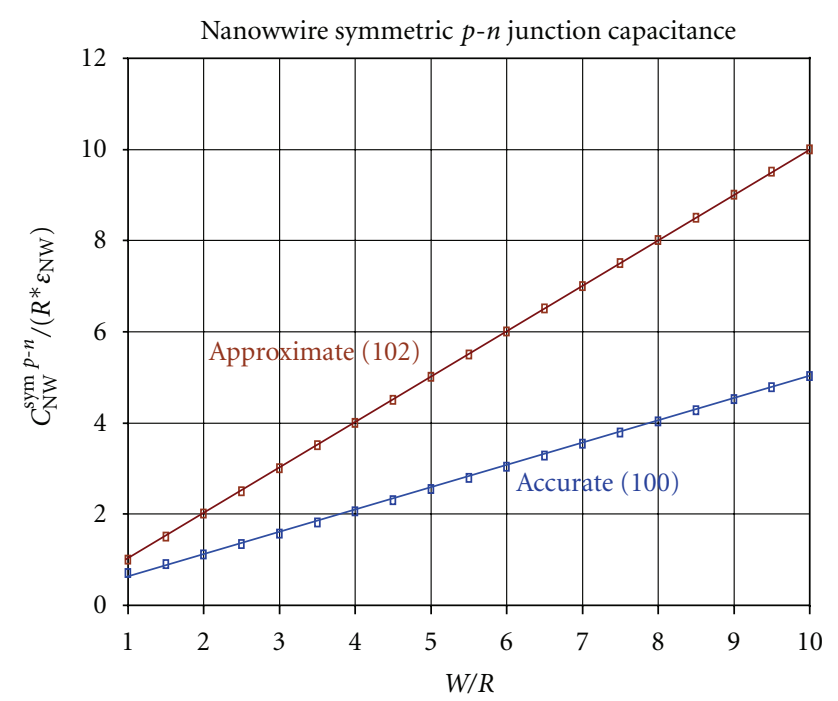

FIgURE 6: Normalized nanowire symmetric $p-n$ semiconductor junction capacitance against $W / R$. Normalization is to $R \varepsilon_{\mathrm{NW}}$. Formulas (100) and (102) providing $C_{\mathrm{NW}}^{p-n}$ are plotted.

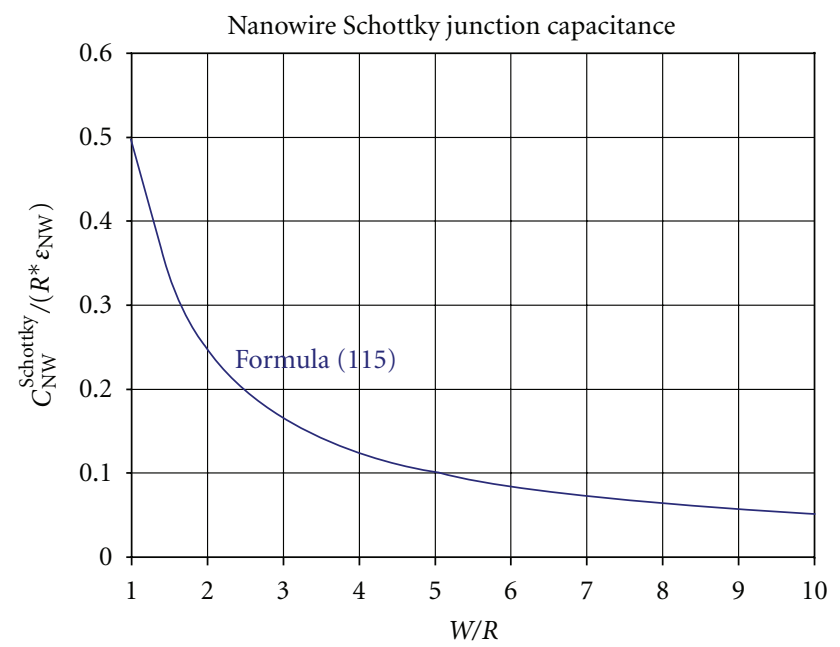

FIgURE 7: Normalized nanowire Schottky junction capacitance against $W / R$. Normalization is to $R \varepsilon_{\mathrm{NW}}$. Formula (115) for $\left.C_{\text {asym }}^{\mathrm{NW}}\right|_{\rho_{p} \rightarrow \infty, W / R \gg 1}$ is plotted.

Figure 7 shows the dependence of the asymmetric nanowire junction capacitance $\left.C_{\mathrm{asym}}^{\mathrm{NW}}\right|_{\rho_{p} \rightarrow \infty, W / R \gg 1}$ on the $W / R$ ratio, as given by (115).

Table 1 summarizes the nanocable capacitance formulas found in the last subsection and in this subsection. The formulas are given in unitless form because each capacitance is normalized to $R \varepsilon_{\mathrm{NW}}$ (this product's units is Farads). The last three rows pertain to the nanowire results.

\section{Discussion and Comparison of Quantum Capacitances and Junction Capacitances}

8.1. Junction Capacitances of Nanocables and Nanowires. Examination of the Schottky nanocable formulas and graphic results plotted shows that although there is a declining dependence with $W / R$ as noted before, it is slower than the simple planar bulk-like behavior seen for $3 \mathrm{D}$ junctions one is familiar with. This arises from the different junction voltage dependence seen in (50), which may be approximated less drastically than (51) to better understand how it controls the roll-off seen in Figure 3. Instead of dropping the second term of (50), but expanding it, we find that

$$
\left.V_{j}^{\mathrm{NC}}\right|_{W \gg \delta}=\rho \frac{2 \pi t_{\mathrm{NC}}}{\varepsilon_{\mathrm{NC}}}\left\{\frac{2 W}{R}+\frac{1}{2}\left(\frac{R}{W}\right)^{2}-1\right\}
$$

giving an effective junction voltage dependence $\left.V_{j}^{\mathrm{NC}}\right|_{W \gg \delta}=$ $\left(4 \pi \rho t_{\mathrm{NC}} / \varepsilon_{\mathrm{NC}}\right)(W / R)^{1+\Delta}$ on $W / R$ which is slightly faster than linear because $\Delta>0$. It is this super linear behavior which causes $C_{\mathrm{NC}}^{\text {Schottky }}$ to decline with $W / R$. Flat dependence for $C_{\mathrm{NC}}^{\text {Schottky }}$ only occurred when the most crude approximation is made, namely, letting the voltage- $-W / R$ ratio be perfectly linear, as in (51).

We also found that an asymmetric $p$ - $n$ nanocable junction, in the limit that the $p$ type doping becomes huge, limits to the just discussed metal-semiconductor junction for nanocables.

Now consider the other Schottky case treated, that for the nanowire. It was studied by considering an asymmetric $p$ - $n$ junction, and then taking the limit as its $p$ side doping approaches a value much in excess of the finite doped $n$ side. The result is (112), a quadratic dependence on $W / R$, which we know gives that classic rapid roll-off in capacitance $C_{\mathrm{NW}}^{\text {Schottky }}$, displayed in Figure 7.

Thus, for both the nanocable and the nanowire, when considering a junction voltage dependence of the form $V_{j}^{\text {Schottky }} \propto(W / R)^{1+\Delta}$, we see that $0<\Delta<1$. One naturally asks how can it be that for these special cases we have some mimicking of 3D behavior. The answer may reside in the fact that the huge charge storage capacity of a nearly infinitely doped side is somewhat equivalent to a planar surface unlimited in its $2 \mathrm{D}$ sheet extent. So a parallel plate capacitor, which is a feature of the abrupt planar 3D junction analysis, may be roughly satisfied for Schottky nanocable and nanowire junctions.

Clearly this is not so for finite doped asymmetric or symmetrically doped nanocable or nanowire junctions. Examination of the exact expressions for voltage-depletion width ratio, in (77) and (97), does not allow one to readily pick out an easily recognizable behavior. However, the approximations allow one to see that logarithmic or modified logarithmic dependences occur in (78) and (98). These dependences cause the capacitances to vary roughly linearly $((81)$ or $(85)$ for the nanocable, and (102) for the nanowire). Therefore, in the form $V_{\text {sym, } j}^{p-n} \propto(W / R)^{1+\Delta}, \Delta<0$ must hold, or the dependence is sublinear. Not surprisingly, the capacitance behavior against $W / R$ does not decline, but rather increases, as seen in Figures 4 and 6.

The increasing capacitances with $W / R$ may arise from the fact that the charge, when changing from a $3 \mathrm{D}$ parallel plate configuration, has in the nanocable or nanowire realizations, had the majority of the infinite sheet folded down and onto a 
TABLE 1: Capacitance formulas for nanocables and nanowires, under different limiting conditions.

\begin{tabular}{|c|c|c|c|}
\hline Formula & Formula $(C /[R \varepsilon])$ & Type & Limit \\
\hline (55) & $\frac{1}{2}\left\{1-0.5 / \sqrt{1+(W / R)^{2}}+(1 / 2)(W / R)^{2} /\left[1+(W / R)^{2}\right]^{3 / 2}\right\}^{-1}$ & $\begin{array}{l}\text { Nanocable- } \\
\text { Schottky }\end{array}$ & $R \gg t_{\mathrm{NC}}$ \\
\hline$(57)$ & $\frac{1}{2}$ & $\begin{array}{l}\text { Nanocable- } \\
\text { Schottky }\end{array}$ & $W \gg R$ \\
\hline$(62)$ & $\frac{1}{4} \frac{R}{W}$ & $\begin{array}{l}\text { Planar Equiv. } \\
\text { NC }\end{array}$ & $W \gg R$ \\
\hline$(81)$ & $\frac{1}{2} \frac{W}{R}$ & $\begin{array}{l}\text { Symmetric } p-n \\
\text { NC }\end{array}$ & $W \gg R$ \\
\hline$(83)$ & $\frac{1}{2} \frac{\sqrt{1+(W / R)^{2}} \sqrt{1+(2 W / R)^{2}}}{2 \sqrt{1+(2 W / R)^{2}}-\sqrt{1+(W / R)^{2}}}$ & $\begin{array}{l}\text { Symmetric } p-n \\
\text { NC }\end{array}$ & $R \gg t_{\mathrm{NC}}$ \\
\hline$(85)$ & $\frac{1}{3} \frac{W}{R}$ & $\begin{array}{l}\text { Symmetric } p-n \\
\text { NC }\end{array}$ & $W \gg R$ \\
\hline$(100)$ & $\frac{1}{4}\left[\sqrt{1+\left(\frac{W}{R}\right)^{2}}-\sqrt{1+\left(\frac{2 W}{R}\right)^{2}}+\frac{(W / R)+(R / W)}{\sqrt{1+(R / W)^{2}}}-\frac{(2 W / R)+(R / 2 W)}{\sqrt{1+(R / 2 W)^{2}}}+\frac{2 W}{R}\right]^{-1}$ & $\begin{array}{l}\text { Symmetric } p-n \\
\text { NW }\end{array}$ & $W \gg R$ \\
\hline$(102)$ & $\frac{W}{R}$ & $\begin{array}{l}\text { Symmetric } p-n \\
\text { NW }\end{array}$ & None \\
\hline$(115)$ & $\frac{1}{2} \frac{R}{W}$ & $\begin{array}{c}\text { Asymmetric } p-n \\
\text { NW }\end{array}$ & $\rho_{p} \rightarrow \infty$ \\
\hline
\end{tabular}

finite extent cylinder. Thus, as junction voltage is increased, the charge is now stored along the cylinder, whereas before it could be placed largely on the infinite sheets. The net effect of this change in charge storage placement, is that no longer does the capacitance provided by a $p-n$ junction appear as a simple parallel plate capacitor with a separation between plates equal to $W$. So instead of the capacitance declining with $W$ as in the simple parallel plate picture, the capacitance may actually go up because of the extra charge swept through when the distance is shifted along the nanocable or nanowire cylinder.

8.2. Intrinsic Quantum Capacitances of Nanocables and Nanowires. There are two widely different perspectives on the configurational placement and consequent charge storage access the nanocables and nanowires will have when considered for energy/charge storage uses such as in fuel cells, batteries, and supercapacitors, versus uses as single or finite numbered electrodes, channels, and other electronic device applications.

In the energy storage/charge storage model, the nanocable or nanowire has direct access to the enveloping medium, which may be a liquid electrolyte, and this provides parallel access charge pathways to the structures. This is not the case, for example, in many electronic devices, for example, when a single nanocylinder is being used as an FET channel, and an electrode below, above, or surrounding it, is acting as the gate. In this case, the nanostructure may be modeled as a cylinder over a ground plane (or back gate), and its classically determined capacitance is $C_{\text {grd-pl }}=\pi \varepsilon / \sinh ^{-1}(2 h / R)$ which for $h \gg R$, is $C_{\text {grd-pl }} \approx \pi \varepsilon / \ln (2 h / R)$ [77, page 1182, (10.1.11), page 1211, (10.1.44)], where $R$ is again merely the cylinder radius, and $h$ is the cylinder center-to-ground separation. One calculates $C_{\text {grd-pl }} \approx 0.005 \mathrm{aF} / \mathrm{nm}$ for $R=$ $0.75 \mathrm{~nm}$ and $h=100 \mathrm{~nm}$ when $\varepsilon=1$. For a much smaller $h=10 \mathrm{~nm}$ when $\varepsilon=4, C_{\text {grd-pl }} \approx 0.034 \mathrm{aF} / \mathrm{nm}$. Comparing this value to the intrinsic quantum capacitance mentioned earlier in Section 2 for single-walled carbon nanotubes, $C_{i}^{\text {SWCNT }}=0.4 \mathrm{aF} / \mathrm{nm}$, shows that $C_{\text {grd-pl }}<$ $C_{i}^{\text {SWCNT }}$. Because the gate potential voltage influencing the nanostructure is in a series circuit (the charge on the gate must flow, even if it is displacement current given by $d D / d t$ in Maxwell's equation, from the gate through the intervening dielectric medium, and then into the nanostructure), the actual capacitive influence of the gate on the nanostructure will be $C_{\text {gate }}=C_{\text {grd-pl }} C_{i}^{\text {SWCNT }} /\left(C_{\text {grd-pl }}+C_{i}^{\text {SWCNT }}\right)$. It is clear that for the selected values for the classical capacitance, $C_{\text {grd-pl }} \ll C_{i}^{\text {SWCNT }}$ and $C_{\text {gate }} \approx C_{\text {grd-pl }}$. That is, the intrinsic quantum capacitance has little effect on the gate-channel biasing relationship.

This may not be true for much larger permittivities, as seen for materials like $\mathrm{HfO}_{2}$, whose dielectric constant is $\varepsilon=16-22$ [78-82], which could push $C_{\text {grd-pl }}$ up to $C_{\text {grd-pl }} \approx 0.187 \mathrm{aF} / \mathrm{nm}$, and then only about a factor of two smaller than $C_{i}^{\text {SWCNT }}$. Another instance where the order of dominance switches, is that of the liquid gating, which has been used to achieve strong field effect action on carbon nanotubes when the nanotube is immersed in solution. The circuit is still essentially one in series, but because some liquids like water (which has $\varepsilon=80$ ) have very large dielectric constants compared to conventional materials like $\mathrm{SiO}_{2}$ or $\mathrm{Si}_{2} \mathrm{~N}_{4}$, this can enormously enhance capacitance. Leonard [14] estimates the capacitance per unit length to be $C_{\lg } \approx$ $10 \mathrm{aF} / \mathrm{nm}$ using the approximation $C_{\mathrm{lg}} \approx 2 \pi \varepsilon / \ln \left(2 l_{V} / R \gamma_{E}\right)$ 
(assumes $R \ll l_{V}$ ), with the potential screening distance in the electrolyte $l_{V}=\sqrt{\varepsilon k_{B} T /\left[Z^{2} e^{2} N_{I}\right]}$, ion valence $Z, N_{I}$ ion density per unit volume, and $\gamma_{E}$ Euler's constant. Reason why $C_{\mathrm{lg}} \approx 10 \mathrm{aF} / \mathrm{nm}$ is so large compared to $C_{\text {grd-pl }}$ is due not only to the large $\varepsilon$, but also because both estimation formulas have logarithmic arguments, with vastly different normalized distances. For $C_{\text {grd-pl }}$, that distance is $2 h$, which we had chosen to be between $200 \mathrm{~nm}$ down to $20 \mathrm{~nm}$, whereas, for $C_{\mathrm{lg}} \approx 10 \mathrm{aF} / \mathrm{nm}$, it is $2 l_{V} / \gamma_{E}$, both normalized with respect to $R$. For water, Leonard indicates $2 l_{V} / \gamma_{E} \approx 1 \mathrm{~nm}$ [14]. With the normalizing distance $R=0.75 \mathrm{~nm}, S_{\text {lg-bg }} \approx$ $\ln (2 h / R) / \ln \left(2 l_{V} / R \gamma_{E}\right)$, making $S_{\text {lg-bg }} \approx \ln (266.7) / \ln (1.333)$, and acknowledging that despite $R \ll l_{V}$ not being well satisfied, going ahead anyway to get the rough estimate $S_{\mathrm{lg}-\mathrm{bg}} \approx 5.586 / 0.2877=19.42$, for the $h=100 \mathrm{~nm}$ case. If one takes the old $C_{\text {grd-pl }} \approx 0.005 \mathrm{aF} / \mathrm{nm}$ value and multiplies by a factor of 80 for permittivity, and 20 for the logarithmic length rescaling, one would obtain 1600 times the old value, giving $C_{\mathrm{lg}} \approx 8 \mathrm{aF} / \mathrm{nm}$. For $h=10 \mathrm{~nm}, S_{\mathrm{lg}-\mathrm{bg}} \approx 3.283 / 0.2877=$ 11.41 , and taking a factor of ten for the logarithmic length rescaling, gives $C_{\mathrm{lg}} \approx 4 \mathrm{aF} / \mathrm{nm}$. Both results show that $C_{\mathrm{lg}}$ is not only much larger than $C_{\text {grd-pl }}$, but considerably in excess of $C_{i}^{\text {SWCNT }}$, making the intrinsic quantum capacitance the controlling storage element in these series types of circuit arrangements found in FET like structures.

\subsection{Various Comparisons of Quantum and Junction Capac-} itances of Nanocables and Nanowires. In order to compare the various intrinsic quantum capacitances with each other, they are plotted against the length over radius ratio in Figure 8 according to (4), (30), and (32) for, respectively, the $\mathrm{RuO}_{2}$ nanowire, the $\mathrm{RuO}_{2} / \mathrm{SiO}_{2}$ nanocable, and the thin walled shell $\mathrm{RuO}_{2} / \mathrm{SiO}_{2}$ nanocable. To plot against $L / R$, each formula must be rescaled to read $C_{i T}=R C_{i}(L / R)$ in aF (attoFarads), and it is this rescaling that causes the nanocable curve to exceed in magnitude (and slope) the nanowire curve. The thin walled nanocable and the singlewalled carbon nanotube (a hollow nanocable) provide stark comparisons, because they are so small and nearly overlap the abscissa. For proper viewing of the SWCNT curve and the thin walled nanocable curve, the results of Figure 8 are replotted in Figure 9 in a $\log _{10}$-linear display. The nearly 4 orders of magnitude variation becomes apparent in the Figure 9 plot.

Similarly to the previous comparison plots between the various intrinsic quantum capacitances, comparison plots of the various junction capacitances are provided in Figure 10. The most accurate formulas (8), (13), (18), and (22), are utilized giving, respectively, the junction capacitances for the Schottky $\mathrm{RuO}_{2} / \mathrm{SiO}_{2}$ nanocable, the symmetric $p$ - $n$ $\mathrm{RuO}_{2} / \mathrm{SiO}_{2}$ nanocable, the symmetric $p-n \mathrm{RuO}_{2}$ nanowire, and the Schottky $\mathrm{RuO}_{2}$ nanowire. To plot against $W / R$, each formula must be rescaled to read $C_{j T}=C_{j}^{n}(R \varepsilon)$ in aF (attoFarads), where the normalized junction capacitances $C_{j}^{n}$ previously plotted are unnormalized here. These curves are then replotted in a $\log _{10}$-linear display in Figure 11, which pulls the Schottky $\mathrm{RuO}_{2}$ nanowire off of the abscissa when it asymptotes.

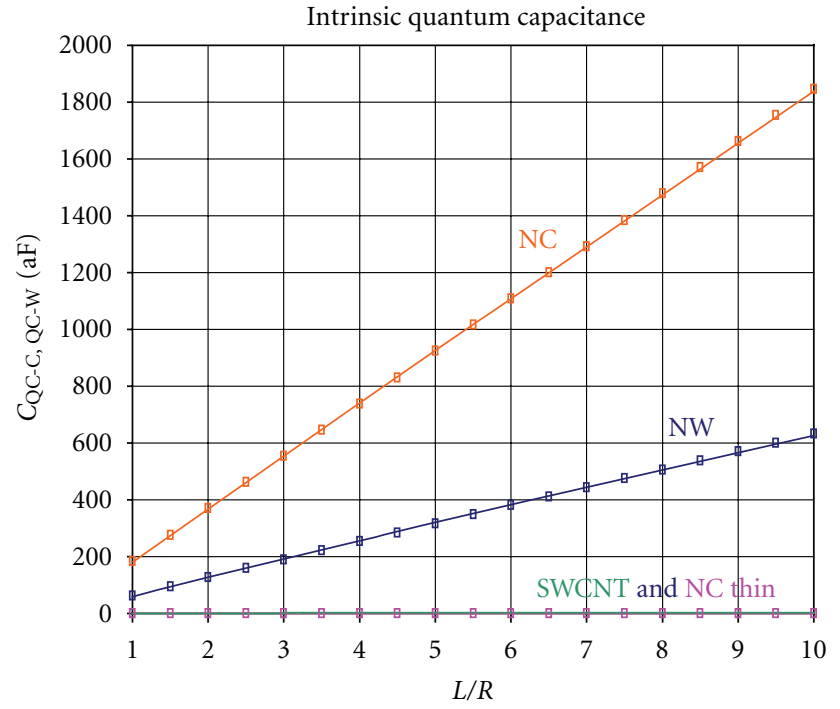

FIgURE 8: Intrinsic quantum capacitance $C_{i T}(\mathrm{aF})$ against $L / R$ according to (4), (30) and (32) and the SWCNT for, respectively, the $\mathrm{RuO}_{2}$ nanowire, the $\mathrm{RuO}_{2} / \mathrm{SiO}_{2}$ nanocable, the thin walled shell $\mathrm{RuO}_{2} / \mathrm{SiO}_{2}$ nanocable, and the carbon nanotube.

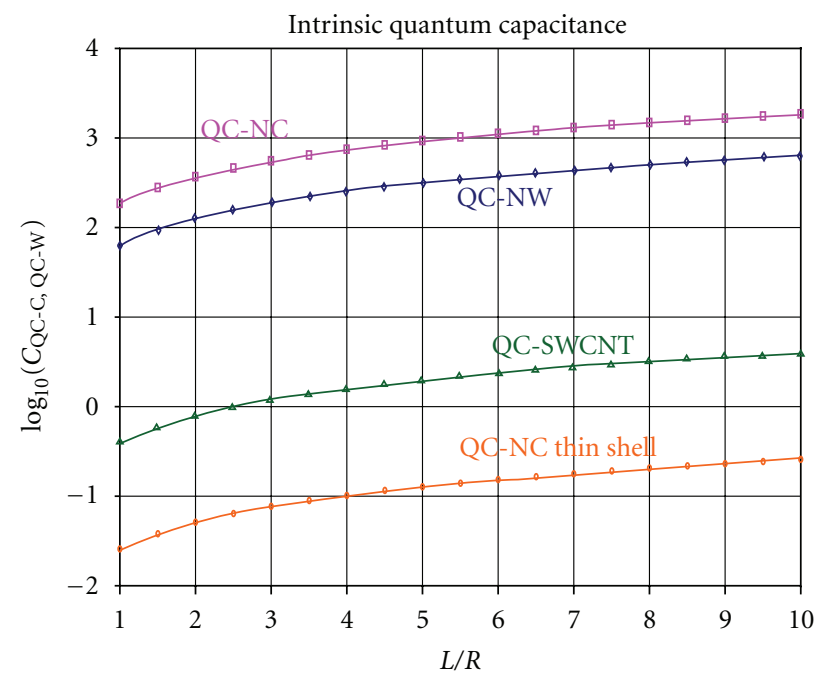

FIGURE 9: Intrinsic quantum capacitance $C_{i T}(\mathrm{aF})$ against $L / R$ curves in Figure 8 replotted in a $\log _{10}$-linear display.

The other reason for replotting the junction capacitance curves on a $\log _{10}$-linear display, is that a direct comparison of the junction capacitances can be made to all of the intrinsic quantum capacitance results. This is accomplished in Figure 12, where all of the intrinsic quantum capacitance results and all of the junction capacitance results are shown together. There is an implicit equivalencing of the length $L$ for the nanostructures used in the intrinsic quantum capacitance calculations and the depletion width $W$ in the junction capacitance calculations, namely, that $L \leftrightarrow W$, allowing one abscissa coordinate to be used. The combined plot shows immediately that the intrinsic quantum capacitance by about one order of magnitude exceeds all of the other capacitances, 


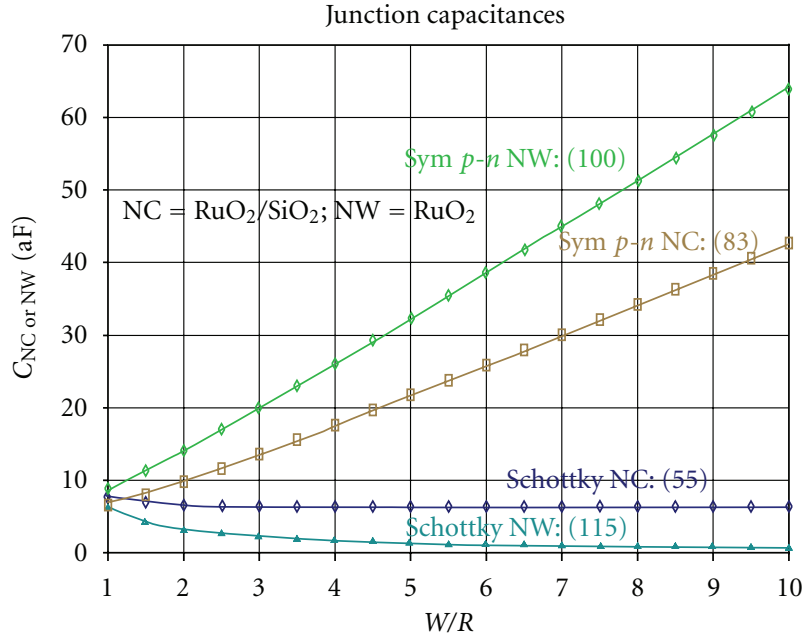

FIgURE 10: Junction capacitance $C_{j}$ against $W / R$ for most accurate formulas (55), (83), (100), and (115) giving, respectively, the results for the Schottky $\mathrm{RuO}_{2} / \mathrm{SiO}_{2}$ nanocable, the symmetric $p-n$ $\mathrm{RuO}_{2} / \mathrm{SiO}_{2}$ nanocable, the symmetric $p-n \mathrm{RuO}_{2}$ nanowire, and the Schottky $\mathrm{RuO}_{2}$ nanowire.

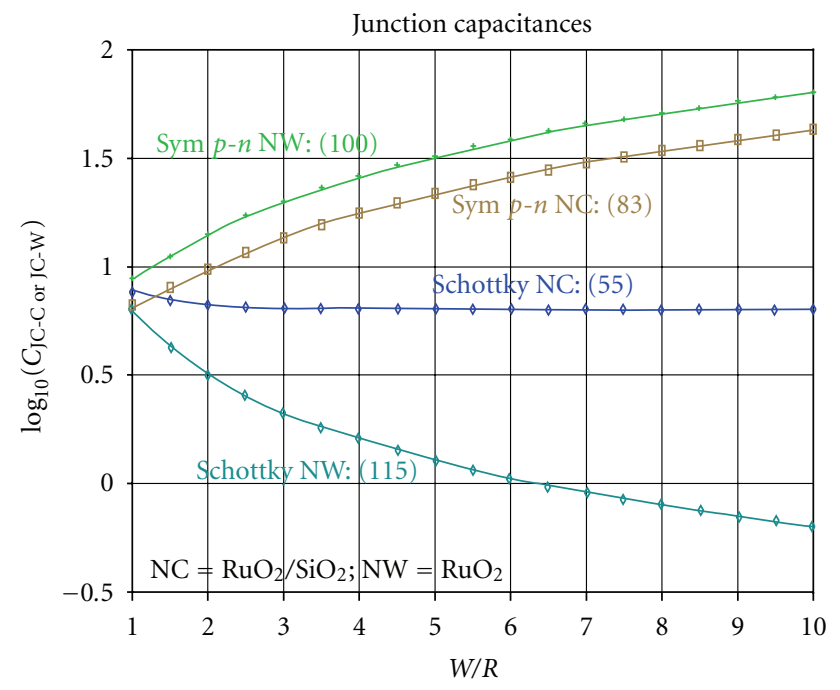

Figure 11: Junction capacitance $C_{j}(\mathrm{aF})$ against $W / R$ curves in Figure 8 replotted in a $\log _{10}$-linear display.

for the considered $\mathrm{RuO}_{2}$ and $\mathrm{RuO}_{2} / \mathrm{SiO}_{2}$ nanostructures. Only for the ultra thin shelled nanocables, namely the SWCNT and the very thin walled $\mathrm{RuO}_{2} / \mathrm{SiO}_{2}$ nanocable, is this not true for the quantum capacitances. For the very thin walled $\mathrm{RuO}_{2} / \mathrm{SiO}_{2}$ nanocable, its quantum capacitance is always less than all of the quantum capacitances and all of the junction capacitances. For the SWCNT quantum capacitance, this is true up to $L / R=W / R=4$, when it crosses the Schottky nanowire junction capacitance, and exceeds it beyond that value, staying below the Schottky nanocable junction capacitance.

8.4. Electrochemistry Meets Physics: Implications for Nanowires and Nanocables. Although we mentioned the $\mathrm{RuO}_{2}$ shell

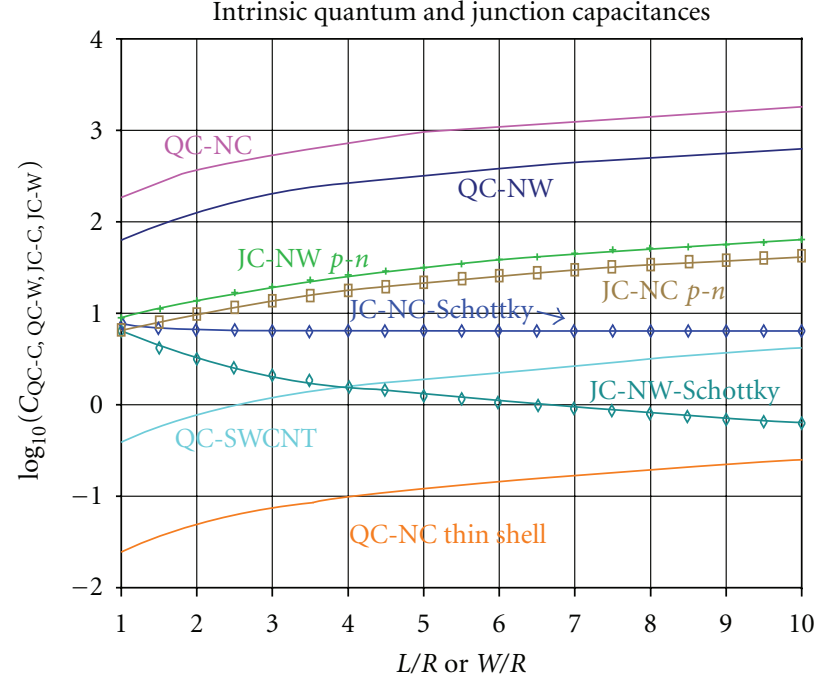

FIgURE 12: Intrinsic quantum capacitance $C_{i T}(\mathrm{aF})$ and junction capacitance $C_{j}(\mathrm{aF})$ replotted in a $\log _{10}$-linear display, against $L / R$ or $W / R$ as a single abscissa.

is anhydrous [30], versus being hydrous [83], and this is sure to affect the potential distributions beyond the coaxial cylinder for the nanocable, and the distributions for a simple continuous core in a $\mathrm{RuO}_{2}$ nanowire, in the past sections the focus has been on the calculation of properties based upon the physics. Here we would like to insert some assessment of possible electrochemical factors, from the perspective of understanding supercapacitors, variously also known as ultracapacitors, and their relation to batteries. For the moment, consider a capacitance $C$ under discussion, to be a constant with voltage. Then, considering the simple arrangement of a parallel plate, as charge is piled up on the plates, with increasing charge accumulated, it becomes increasingly harder to add additional charge. This is why the energy stored on the capacitor will be $E_{\text {cap }}=C V^{2} / 2=Q V / 2$, with the factor of $1 / 2$ included, for $V$ being the final potential difference on the plates. However, this is not the case for the electrochemical charging of a cell in a battery obeying a Nerstian relation. For the battery, $E_{\text {batt }}=C V^{2}=Q V$, and this relation was obtained noting the fact that for an ideal battery, the voltage on the cell remains constant as more charge is accumulated [84].

If $C$ is not a constant, then it is suggested that $C=d Q / d V$ instead of $C=Q / V$, which may occur in the case of doublelayer or pseudocapacitance at electrodes [84]. The doublelayer capacitance $C_{d l}$ ubiquitously arises at all electrode interfaces, having higher values for aqueous electrolytes than for nonaqueous solvents or organic surfactants. The doublelayer capacitance values can be quite large, and are caused by the small double-layer charge separations on the order of $3 \AA$ for a compact double-layer. Extremely diffuse doublelayers, on the other hand, can have charge distributions over an extent of $0.1 \mu \mathrm{m}$. Utilization of the double-layer at electrodes, with typical values $15-50 \mu \mathrm{F} / \mathrm{cm}^{2}$, can result in very large capacitive densities of $250 \mathrm{~F} / \mathrm{g}$, using an effective area per gram of $1000 \mathrm{~m}^{2} / \mathrm{g}$ as for carbon, for example. This 


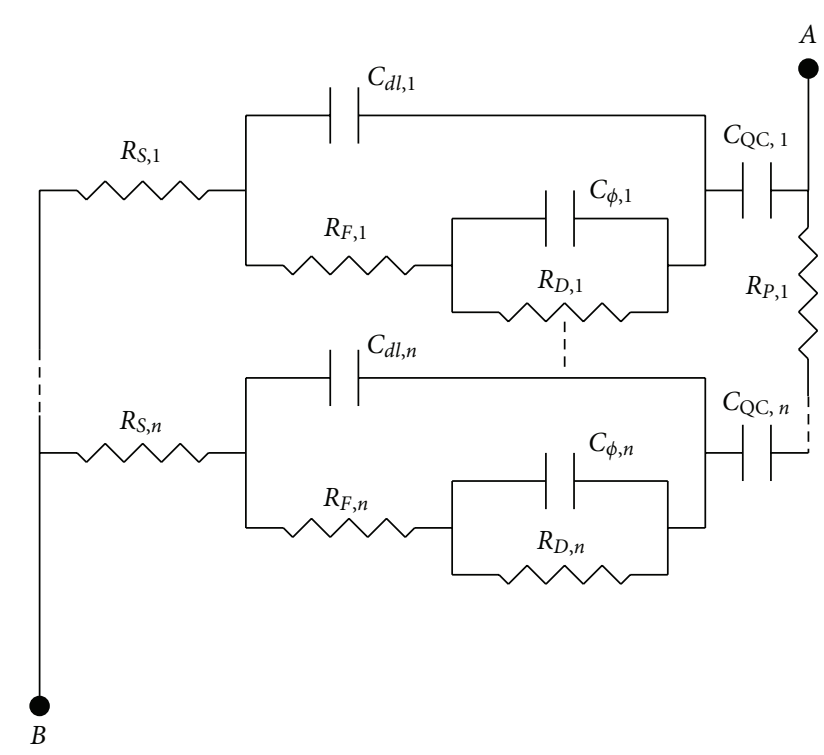

Figure 13: Circuit diagram of the intrinsic quantum capacitance embedded in the double-layer/pseudocapacitive subcircuit.

is consistent with the large values seen for anhydrous or hydrous $\mathrm{RuO}_{2}$ (whose value can be 3 times larger). The double-layer capacitance is affiliated with electrostatic charge separation.

Pseudocapacitance is distinguished from double-layer capacitance by its origin-it arises in cases where Faradaic charge transfer processes lead to passage of charge that depend on thermodynamic factors and the potential. Conway et al. [84] designate this capacitance as $C_{\phi}$, and associate it with redox reactions for which the potential is a logarithmic function of the ratio of activities of the oxidized and reduced species, or with a process of progressive occupation of the surfaces sites on an electrode by underpotentialdeposited species.

In view of Section 8.2, here is proposed a circuit model for an extended length nanowire or nanocable, or an electrode made thereof, based upon the equivalent circuits in Sections 3.2-3.4 of [84]. In the circuit diagram, shown in Figure 13, we see the intrinsic quantum capacitance, denoted by $C_{\mathrm{QC}}$, linked on the porous nanostructure by a resistance $R_{p}$. The quantum capacitances come off of this trunk, pointing perpendicular to it, branching in a series fashion, into a parallel arrangement of the doublelayer $C_{d l}$ and pseudo $C_{\phi}$-capacitances. Resistances $R_{F}$ and $R_{D}$ are, respectively, the Faradaic charging and discharging resistances. $R_{D}$ and $C_{\phi}$ are in parallel, in series with $R_{F}$, and the entire parallel arrangement in series with the solution resistance $R_{S}$. One terminal $B$, is connected off of these solution resistances, whereas the other terminal $A$ is attached to the nanostructures. One may view the previously discussed liquid gating capacitance $C_{\mathrm{lg}}$ in Section 8.2, as inserted in place of the double-layer capacitance $C_{\mathrm{dl}}$, or the pseudocapacitance $C_{\phi}$, depending upon the specific electrochemical interactions taking place.

\section{Conclusions}

We have derived and calculated the intrinsic quantum capacitances and transmission coefficients based upon invoking $\mathrm{ab}$ initio first principle density of states values. These capacitances are on the order of attofarads ( $\mathrm{aF}$ 's), and have been found for $\mathrm{RuO}_{2}$ nanowires $(63 \mathrm{aF})$ and $\mathrm{RuO}_{2} / \mathrm{SiO}_{2}$ nanocables $(6 \mathrm{aF})$. Comparison to single-walled carbon nanotubes (SWCNTs) has been made $(0.4 \mathrm{aF})$. We have also calculated the Schottky, unsymmetric and symmetric junction capacitances of nanocables, and evaluated the formulas for both SWCNTs $\left(0.06 \mathrm{aF}\right.$ for doping exceeding $10^{20} / \mathrm{cc}$ of a $1 \mathrm{~nm}$ tube radius) and $\mathrm{RuO}_{2} / \mathrm{SiO}_{2}$ nanocables $(6 \mathrm{aF}$ for a $30 \mathrm{~nm}$ inner dielectric core radius). $\mathrm{RuO}_{2}$ and $\mathrm{CNT}$ nanowire capacitances have been calculated for symmetric and unsymmetric $p-n$ junctions, and found to be as low as $0.024 \mathrm{aF}$ for doping exceeding $10^{18} / \mathrm{cc}$ (and a $1 \mathrm{~nm}$ radius) and as high as $13 \mathrm{aF}$ for a $30 \mathrm{~nm}$ nanowire radius. (Note that the results here were more carefully and thoroughly developed than in two earlier works [85] and [86, (53) and (57) are incorrect, and are correctly stated here as (97) and (101)], with entirely new aspects presented for the first time.)

With the continuing current pursuit of employing nanotubes [87-91], nanowires $[92,93]$ and including $\mathrm{V}_{3} \mathrm{O}_{7} \cdot \mathrm{H}_{2} \mathrm{O}$ nanowires and $\mathrm{Si} / \mathrm{a}-\mathrm{Si}$ core/shells or nanocables [94], and nanocables [95] in various components from electronics (diodes, transistors, photodetectors, and photovoltaic cells) to chemistry (sensors, membranes, catalysts, batteries, fuel cells, and supercapacitors), there is little doubt that knowledge of the intrinsic quantum capacitances obtained here will be useful. That is also true for the fundamental junction capacitances found here for these nanoscopic structures. In fact, the idea of obtaining high energy and charge storage is very much uppermost in the minds of researchers and technologists these days, as is readily evidenced, for example, by the recent result on supercapacitors fabricated using SWCNTs [96]. This trend of enhancing capacitance in terms of its uses and quantitative charge and energy storage is sure to endure in the future.

\section{Appendices}

\section{A. Green's Function Solution for Poisson's Equation: The Electrostatic Limit}

Poisson's equation, giving the effect of a medium to a charge distribution, is expressed as

$$
\nabla \cdot \nabla \varphi=-\frac{\rho}{\varepsilon}
$$

where the vacuum permittivity $\varepsilon_{0}$ seen in [63] (uses mks units) has been upgraded to be a general value. The potential $\varphi$ and electric field $\mathbf{E}$ are related by

$$
\mathbf{E}=-\nabla \varphi
$$

in the electrostatic limit to Maxwell's equations. (In the Lorentz gauge, the dropped term is proportional to $\mu \partial \mathrm{A} / \partial t$.) 
By the Helmholtz theorem, a vector $\mathbf{V}_{\mathbf{g}}$ may be written in terms of a divergence part and a curl part,

$$
\mathbf{V}_{\mathbf{g}}=-\nabla \varphi+\nabla \times \mathbf{A}
$$

where the electrostatic $\varphi$ and vector potentials $\mathbf{A}$ are be given by

$$
\begin{aligned}
& \varphi(\mathbf{r})=\frac{1}{4 \pi} \int \frac{s\left(\mathbf{r}^{\prime}\right)}{R_{s f}} d \tau^{\prime}, \\
& \mathbf{A}(\mathbf{r})=\frac{1}{4 \pi} \int \frac{\mathbf{c}\left(\mathbf{r}^{\prime}\right)}{R_{s f}} d \tau^{\prime}
\end{aligned}
$$

with $s\left(\mathbf{r}^{\prime}\right)$ and $\mathbf{c}\left(\mathbf{r}^{\prime}\right)$ being

$$
\begin{aligned}
& s\left(\mathbf{r}^{\prime}\right)=\left.\nabla \cdot \mathbf{V}\right|_{\mathbf{r}^{\prime}}, \\
& \mathbf{c}\left(\mathbf{r}^{\prime}\right)=\nabla \times\left.\mathbf{V}\right|_{\mathbf{r}^{\prime}}
\end{aligned}
$$

In (A.4a), (A.4b) equations, $R_{s f}$ is the distance between the source and field points located at, respectively, $\mathbf{r}^{\prime}$ and $\mathbf{r}$.

Notice that

$$
\nabla_{\mathbf{r}} \cdot \mathbf{V}\left(\mathbf{r}, \mathbf{r}^{\prime}\right)=-\frac{\rho\left(\mathbf{r}^{\prime}\right)}{\mathcal{E}\left(\mathbf{r}^{\prime}\right)}
$$

in $\mathbf{r}$ coordinate space for the field point, will be in $\mathbf{r}^{\prime}$ coordinate space, with $\nabla_{\mathbf{r}}=-\nabla_{\mathbf{r}^{\prime}}$,

$$
\nabla_{\mathbf{r}^{\prime}} \cdot \mathbf{V}=\frac{\rho\left(\mathbf{r}^{\prime}\right)}{\mathcal{E}\left(\mathbf{r}^{\prime}\right)}
$$

and for permittivity being uniform, reduces to

$$
\nabla_{\mathbf{r}^{\prime}} \cdot \mathbf{V}=\frac{1}{\varepsilon} \rho\left(\mathbf{r}^{\prime}\right)
$$

If this is inserted into (A.4a) for $\varphi\left(\mathbf{r}^{\prime}\right)$, we find

$$
\varphi(\mathbf{r})=\frac{1}{4 \pi \varepsilon} \int \frac{\rho\left(\mathbf{r}^{\prime}\right)}{R_{s f}} d \tau^{\prime} .
$$

A Green's function for electrostatic potential $\varphi_{g}$ is defined through the equation

$$
\nabla \cdot \nabla \varphi_{g}=-\delta\left(\mathbf{r}-\mathbf{r}^{\prime}\right) .
$$

However, it is known that the $\nabla^{2}$ operator acting on $R_{s d}^{-1}$ yields

$$
\nabla^{2}\left(\frac{1}{R_{s d}}\right)=-4 \pi \delta\left(\mathbf{r}-\mathbf{r}^{\prime}\right)
$$

making $\varphi_{g}$ satisfy

$$
\varphi_{g}\left(\mathbf{r}, \mathbf{r}^{\prime}\right)=4 \pi G\left(\mathbf{r}, \mathbf{r}^{\prime}\right)=\frac{1}{R_{s f}}
$$

where $G\left(\mathbf{r}, \mathbf{r}^{\prime}\right)$ is merely the electrostatic Green's function. That is, $G\left(\mathbf{r}, \mathbf{r}^{\prime}\right)$ satisfies

$$
\nabla^{2}\left[G\left(\mathbf{r}, \mathbf{r}^{\prime}\right)\right]=-\delta\left(\mathbf{r}-\mathbf{r}^{\prime}\right),
$$

with

$$
G\left(\mathbf{r}, \mathbf{r}^{\prime}\right)=\frac{1}{4 \pi R_{s f}}
$$

This is the bare Green's function which is missing the permittivity. How it appears, becomes apparent when substituting (A.14) into (A.9):

$$
\begin{aligned}
\varphi(\mathbf{r}) & =\frac{1}{\varepsilon} \int G\left(\mathbf{r}, \mathbf{r}^{\prime}\right) \rho\left(\mathbf{r}^{\prime}\right) d \tau^{\prime} \\
& =\int\left[\frac{1}{\varepsilon} G\left(\mathbf{r}, \mathbf{r}^{\prime}\right)\right] \rho\left(\mathbf{r}^{\prime}\right) d \tau^{\prime} \\
& =\int G_{e s}\left(\mathbf{r}, \mathbf{r}^{\prime}\right) \rho\left(\mathbf{r}^{\prime}\right) d \tau^{\prime}
\end{aligned}
$$

This gives the final form of the desired electrostatic Green's function,

$$
G_{e s}\left(\mathbf{r}, \mathbf{r}^{\prime}\right)=\frac{1}{\varepsilon} G\left(\mathbf{r}, \mathbf{r}^{\prime}\right)=\frac{1}{4 \pi \varepsilon} \frac{1}{\left|\mathbf{r}-\mathbf{r}^{\prime}\right|}
$$

Equation (A.16) is in mks units and is converted to cgs units by replacing the permittivity by $\mathcal{E} /(4 \pi)$, the form used in the text.

\section{B. Nonabrupt Nanocable Junctions}

This discussion is based upon Leonard [14], with amendments, and recognizes that although an abrupt assumption for a junction is a reasonable starting point, more accurate but more involved analytical approaches may be possible, when treating the charge distribution and its affect on the potential variation with $z$. The charge distribution's affect on the potential $V(z)$ has been indirectly acknowledged in (48) for the nanocable junction voltage $V_{j}^{\mathrm{NC}}$ where mobile majority carrier spatial distribution thermal tail is taken into account by the additive term $-\alpha_{\mathrm{NC}} k_{B} T / e$. Here we will extend the discussion to include explicitly the affect on $V(z)$ itself. Consider the limiting asymmetric case when doping is equal on both sides of the nanocable junction, $z_{n}=z_{p}=W$, making (66) become

$$
\rho_{\mathrm{sym}}^{\mathrm{NC}}(z)= \begin{cases}\rho, & 0<z<W, \\ \alpha V(z), & z>W \\ -\rho, & -W<z<0, \\ \alpha V(z), & z<-W .\end{cases}
$$

(This charge distribution $\rho(z)$ is not unlike that utilized in (85) for the nanowire case, but without the added linear regions for $|z|>W$.)

Following (A.15), (A.16), and (39) (first line), using the kernel for the nanocable (its Green's function) in (38), nanocable potential is

$$
V_{\mathrm{NC}}(z)=\int_{-\infty}^{\infty} K\left(z, z^{\prime}\right) \rho_{\mathrm{sym}}^{\mathrm{NC}}\left(z^{\prime}\right) d z^{\prime} .
$$


This potential solution may be broken up into three charge distribution contribution regions, as evidenced by (B.1), leading to $\left(\rho_{l}(z)=\rho_{\mathrm{sym}}^{\mathrm{NC}}(z)\right.$, the line charge distribution)

$$
\begin{aligned}
V_{\mathrm{NC}}(z)= & \int_{-\infty}^{-W} K\left(z, z^{\prime}\right) \rho_{l}\left(z^{\prime}\right) d z^{\prime}+\int_{-W}^{W} K\left(z, z^{\prime}\right) \rho_{l}\left(z^{\prime}\right) d z^{\prime} \\
& +\int_{W}^{\infty} K\left(z, z^{\prime}\right) \rho_{l}\left(z^{\prime}\right) d z^{\prime} \\
= & V_{0}(z)+\int_{-\infty}^{-W} K\left(z, z^{\prime}\right) \rho_{l}\left(z^{\prime}\right) d z^{\prime} \\
& +\int_{W}^{\infty} K\left(z, z^{\prime}\right) \rho_{l}\left(z^{\prime}\right) d z^{\prime} \\
\approx & V_{0}(z) \\
& +\alpha\left[\int_{-\infty}^{-W} V_{\mathrm{NC}}\left(z^{\prime}\right) K\left(z, z^{\prime}\right) d z^{\prime}\right. \\
& \left.+\int_{W}^{\infty} V_{\mathrm{NC}}\left(z^{\prime}\right) K\left(z, z^{\prime}\right) d z^{\prime}\right]
\end{aligned}
$$

where $V_{0}(z)$ is defined as

$$
V_{0}(z)=\int_{-W}^{W} K\left(z, z^{\prime}\right) \rho_{l}\left(z^{\prime}\right) d z^{\prime}
$$

the depletion width generated potential distribution. Define the potential integral contribution outside of the depletion region as

$$
V_{W}(z)=\int_{-\infty}^{-W} K\left(z, z^{\prime}\right) \rho_{l}\left(z^{\prime}\right) d z^{\prime}+\int_{W}^{\infty} K\left(z, z^{\prime}\right) \rho_{l}\left(z^{\prime}\right) d z^{\prime}
$$

and its approximation as

$V_{W}^{\alpha}(z) \approx \alpha\left[\int_{-\infty}^{-W} V_{\mathrm{NC}}\left(z^{\prime}\right) K\left(z, z^{\prime}\right) d z^{\prime}+\int_{W}^{\infty} V_{\mathrm{NC}}\left(z^{\prime}\right) K\left(z, z^{\prime}\right) d z^{\prime}\right]$

An approximation to $V(z)$ over the whole domain $[-\infty, \infty]$, employing (B.6), looks like

$V_{\mathrm{NC}}^{\alpha}(z)$

$$
\begin{aligned}
= & \left.\int_{-\infty}^{\infty} K\left(z, z^{\prime}\right) \rho_{l}\left(z^{\prime}\right) d z^{\prime}\right|_{\rho_{l}\left(z^{\prime}\right)=\alpha V\left(z^{\prime}\right)} \\
= & \alpha \int_{-\infty}^{\infty} V_{\mathrm{NC}}\left(z^{\prime}\right) K\left(z, z^{\prime}\right) d z^{\prime} \\
= & \alpha \int_{-W}^{W} V_{\mathrm{NC}}\left(z^{\prime}\right) K\left(z, z^{\prime}\right) d z^{\prime} \\
& +\alpha\left[\int_{-\infty}^{-W} V_{\mathrm{NC}}\left(z^{\prime}\right) K\left(z, z^{\prime}\right) d z^{\prime}+\int_{W}^{\infty} V_{\mathrm{NC}}\left(z^{\prime}\right) K\left(z, z^{\prime}\right) d z^{\prime}\right] \\
= & \alpha \int_{-W}^{W} V_{\mathrm{NC}}\left(z^{\prime}\right) K\left(z, z^{\prime}\right) d z^{\prime}+V_{W}^{\alpha}(z)
\end{aligned}
$$

by (B.6). Inserting (B.7) into the expression (B.3) yields

$$
\begin{aligned}
& V_{\mathrm{NC}}(z) \\
& \approx V_{0}(z)+V_{W}^{\alpha}(z) \\
& =V_{0}(z)+V_{\mathrm{NC}}^{\alpha}(z)-\alpha \int_{-W}^{W} V_{\mathrm{NC}}\left(z^{\prime}\right) K\left(z, z^{\prime}\right) d z^{\prime} \\
& =\left[V_{0}(z)-\alpha \int_{-W}^{W} V_{\mathrm{NC}}\left(z^{\prime}\right) K\left(z, z^{\prime}\right) d z^{\prime}\right]+V_{\mathrm{NC}}^{\alpha}(z) .
\end{aligned}
$$

Defining

$$
V_{0}^{\alpha}(z)=V_{0}(z)-\alpha \int_{-W}^{W} V_{\mathrm{NC}}\left(z^{\prime}\right) K\left(z, z^{\prime}\right) d z^{\prime}
$$

allows (B.8) to be cast as

$$
V_{\mathrm{NC}}(z) \approx V_{0}^{\alpha}(z)+V_{\mathrm{NC}}^{\alpha}(z)
$$

with

$$
V_{\mathrm{NC}}^{\alpha}(z)=\alpha \int_{-\infty}^{\infty} V_{\mathrm{NC}}\left(z^{\prime}\right) K\left(z, z^{\prime}\right) d z^{\prime} .
$$

Putting these last two equations together gives a single integral equation for $V_{\mathrm{NC}}(z)$,

$$
V_{\mathrm{NC}}(z) \approx V_{0}^{\alpha}(z)+\alpha \int_{-\infty}^{\infty} V_{\mathrm{NC}}\left(z^{\prime}\right) K\left(z, z^{\prime}\right) d z^{\prime} .
$$

This integral equation may be solved by defining a Fourier transform pair:

$$
\begin{gathered}
\tilde{V}_{\mathrm{NC}}(q)=\int_{-\infty}^{\infty} V_{\mathrm{NC}}(z) e^{-i q z} d z, \\
V_{\mathrm{NC}}(z)=\frac{1}{2 \pi} \int_{-\infty}^{\infty} \tilde{V}_{\mathrm{NC}}(q) e^{i q z} d q
\end{gathered}
$$

taking the Fourier transform of (B12), we have

$$
\begin{aligned}
& \tilde{V}_{\mathrm{NC}}(q) \\
& \approx \tilde{V}_{0}^{\alpha}(q)+\alpha \int_{-\infty}^{\infty} V_{\mathrm{NC}}\left(z^{\prime}\right) d z^{\prime} \int_{-\infty}^{\infty} e^{-i q z} K\left(z-z^{\prime}\right) d z \\
& =\tilde{V}_{0}^{\alpha}(q)+\alpha \int_{-\infty}^{\infty} V_{\mathrm{NC}}\left(z^{\prime}\right) d z^{\prime} e^{-i q z^{\prime}} \tilde{K}(q) \\
& =\tilde{V}_{0}^{\alpha}(q)+\alpha \tilde{K}(q) \int_{-\infty}^{\infty} V_{\mathrm{NC}}\left(z^{\prime}\right) d z^{\prime} e^{-i q z^{\prime}} \\
& =\tilde{V}_{0}^{\alpha}(q)+\alpha \tilde{K}(q) \tilde{V}_{\mathrm{NC}}(q),
\end{aligned}
$$

where the space-shifting property of the Fourier transform is invoked [97]. (It also follows from the space-convolution property.) In (B.14) we used the Green's function behavior that $K\left(z, z^{\prime}\right)=K\left(z-z^{\prime}\right)$. Because (B.14) is an algebraic statement, it allows nanocable potential solution by simple manipulation,

$$
\tilde{V}_{\mathrm{NC}}(q) \approx \frac{\tilde{V}_{0}^{\alpha}(q)}{1-\alpha \tilde{K}(q)}
$$


The Fourier transform of the kernel is determined as follows. Enlisting (38) for $K\left(z, z^{\prime}\right)$, and a similar transform pair to (B.13),

$$
\begin{aligned}
\widetilde{K}(q) & =\frac{2 \pi R t_{\mathrm{NC}}}{\varepsilon_{\mathrm{NC}}} \int_{-\infty}^{\infty} \frac{e^{-i q z}}{\sqrt{R^{2}+z^{2}}} d z \\
& =\frac{2 \pi R t_{\mathrm{NC}}}{\varepsilon_{\mathrm{NC}}} \int_{-\infty}^{\infty} \frac{[\cos (q z)-i \sin (q z)]}{\sqrt{R^{2}+z^{2}}} d z \\
& =\frac{4 \pi R t_{\mathrm{NC}}}{\varepsilon_{\mathrm{NC}}} \int_{0}^{\infty} \frac{\cos (q z)}{\sqrt{R^{2}+z^{2}}} d z \\
& =\frac{4 \pi R t_{\mathrm{NC}}}{\varepsilon_{\mathrm{NC}}} \int_{0}^{\infty} \frac{\cos (q R \bar{z})}{\sqrt{1+\bar{z}^{2}}} d \bar{z} \\
& =\frac{4 \pi R t_{\mathrm{NC}}}{\varepsilon_{\mathrm{NC}}} K_{0}(q R),
\end{aligned}
$$

where the variable $\bar{z}=z / R$ and $K_{0}$ is the modified Bessel function of integer order $n=0$ [98, page 376, 9.6.21 for $q R>0$ ]. For the small $K_{0}$ argument limit, an approximation derivable from small argument limits of the Bessel functions of the first and second kinds, $J_{0}$ and $N_{0}$, may be found utilizing [99], $K_{0}(u) \approx-\ln (\gamma u / 2) \approx-\ln (u)$, reducing $\widetilde{K}(q)$ in (B.16) to

$$
\tilde{K}(q) \approx \frac{4 \pi R t_{\mathrm{NC}}}{\varepsilon_{\mathrm{NC}}} \ln (q R) .
$$

This is a reasonable argument when thinking about large $z$ values.

Nanocable Fourier transform potential $\widetilde{V}_{\mathrm{NC}}(q)$ evaluation by (B.15) necessitates obtaining the Fourier transform of $\tilde{V}_{0}^{\alpha}(q)$. If one takes $\tilde{V}_{0}(q)$ as a first approximation to $\tilde{V}_{0}^{\alpha}(q)$, then in principle $\widetilde{V}_{\mathrm{NC}}(q)$ can be found from the simplified form of (B.15),

$$
\tilde{V}_{\mathrm{NC}}(q) \approx \frac{\tilde{V}_{0}(q)}{1-\alpha \tilde{K}(q)},
$$

where $\tilde{V}_{0}(q)$ is

$$
\widetilde{V}_{0, \mathrm{NC}}(q)=\int_{-\infty}^{\infty} e^{-i q z}\left\{\begin{array}{c}
V_{0, \mathrm{NC}}^{\text {Schottky }}(z) \\
V_{0, \mathrm{NC}}^{\text {asy } p \text {-n }}(z)
\end{array}\right\} d z .
$$

$V_{0, \mathrm{NC}}^{\text {Schottky }}$ and $V_{0, \mathrm{NC}}^{\text {asy } p-n}$ are given in, respectively, in (44) and (71). Equation (B.19) can be rewritten as

$$
\widetilde{V}_{0, \mathrm{NC}}(q)=\rho \frac{2 \pi R t_{\mathrm{NC}}}{\varepsilon_{\mathrm{NC}}} \int_{-\infty}^{\infty} e^{-i q z}\left\{\begin{array}{c}
I_{0, \mathrm{NC}}^{\text {Schottky }}(z) \\
I_{0, \mathrm{NC}}^{\text {asy } p-n}(z)
\end{array}\right\} d z .
$$

Simplified forms of $I_{0, \mathrm{NC}}^{\text {Schottky }}(z)$ and $I_{0, \mathrm{NC}}^{\text {asy }}$-n $(z)$ exist for large $z$, recalling $(45)$ for the Schottky case, making $I_{0, \mathrm{NC}}^{\text {Schotky }}(z)=$ $-\{1+\delta / W\}(W / z)^{2}$, and using the knowledge that for symmetric limit $p-n$ junction case of (71) has

$$
V_{\mathrm{NC}}(z) \approx \rho \frac{4 \pi R t_{\mathrm{NC}}}{\varepsilon_{\mathrm{NC}}}\left(\frac{W}{z}\right)^{2}
$$

its real space integral to be inserted into (B.20) is

$$
\left.I_{0, \mathrm{NC}}^{\mathrm{asym}}(z)\right|_{\rho_{n}=\rho_{p}}=I_{0, \mathrm{NC}}^{\mathrm{asym}}(z)=2\left(\frac{W}{z}\right)^{2} .
$$

Placing these last expressions into (B.20) provides the formula

$$
\begin{aligned}
\tilde{V}_{0, \mathrm{NC}}(q) & \rho \frac{2 \pi R t_{\mathrm{NC}}}{\varepsilon_{\mathrm{NC}}}\left\{\left\{1+\frac{\delta}{W}\right\}\right\} \int_{-\infty}^{\infty} e^{-i q z}\left(\frac{W}{z}\right)^{2} d z \\
= & \rho \frac{2 \pi R t_{\mathrm{NC}}}{\varepsilon_{\mathrm{NC}}}\left\{\left\{1+\frac{\delta}{W}\right\}\right\} \\
& \times \int_{-\infty}^{\infty}[\cos (q z)-i \sin (q z)]\left(\frac{W}{z}\right)^{2} d z \\
= & \rho \frac{4 \pi R t_{\mathrm{NC}}}{\varepsilon_{\mathrm{NC}}}\left\{\left\{1+\frac{\delta}{W}\right\}\right\} \int_{0}^{\infty} \cos (q z)\left(\frac{W}{z}\right)^{2} d z \\
= & \rho \frac{4 \pi R t_{\mathrm{NC}}}{\varepsilon_{\mathrm{NC}}} W^{2} q\left\{\left\{1+\frac{\delta}{W}\right\}\right\} \int_{0}^{\infty} \frac{\cos (\bar{z})}{\bar{z}} d \bar{z}
\end{aligned}
$$

using the normalization $\bar{z}=q z$. If one takes $W \approx 1 / q$ in (B23), then it becomes

$$
\widetilde{V}_{0, \mathrm{NC}}(q)=\rho \frac{4 \pi R t_{\mathrm{NC}}}{\varepsilon_{\mathrm{NC}}} \frac{1}{q}\left\{\left\{1+\frac{\delta}{W}\right\}\right\} I_{c n},
$$

where the $I_{c n}$ integral is

$$
I_{c n}=\int_{0}^{\infty} \frac{\cos (\bar{z})}{\bar{z}} d \bar{z}
$$

Clearly this integral is not well posed, as it has a troublesome singularity at $z=0$. This arose from making many simplifications in arriving at its final simplified form.

The value of the highly simplified form of (B.25) is that it demonstrates that the form of $\tilde{V}_{0}(q)$ is roughly an odd 
function of $q$, which allows $V_{\mathrm{NC}}(z)$ to be obtained from the inverse Fourier transform of (B.18) in a direct manner,

$$
\begin{aligned}
& V_{\mathrm{NC}}(z)=\frac{1}{2 \pi} \int_{-\infty}^{\infty} \tilde{V}_{\mathrm{NC}}(q) e^{i q z} d q \\
& =\frac{1}{2 \pi} \int_{-\infty}^{\infty} \tilde{V}_{\mathrm{NC}}(q)[\cos (q z)+i \sin (q z)] d q \\
& =\frac{i}{\pi} \int_{0}^{\infty} \tilde{V}_{\mathrm{NC}}(q) \sin (q z) d q \\
& \approx \frac{i}{\pi} \int_{0}^{\infty} \frac{\tilde{V}_{0}^{\alpha}(q)}{1-\alpha \tilde{K}(q)} \sin (q z) d q \\
& \approx-\frac{i}{\pi \alpha} \int_{0}^{\infty} \frac{\tilde{V}_{0}^{\alpha}(q)}{\tilde{K}(q)} \sin (q z) d q \\
& \approx-\frac{i \rho}{\pi \alpha} I_{c n}\left\{\left\{1+\frac{\delta}{W}\right\}\right\} \int_{0}^{\infty} \frac{\sin (q z)}{q \ln (q R)} d q \\
& \approx-\frac{i \rho}{\pi \alpha} I_{c n}\left\{\left\{1+\frac{\delta}{W}\right\}\right\} \int_{0}^{q_{c}} \frac{\sin (q z)}{q \ln (q R)} d q \\
& \approx-\frac{i \rho}{\pi \alpha} I_{c n}\left\{\left\{1+\frac{\delta}{W}\right\}\right\} z \int_{0}^{q_{c}} \frac{1}{\ln (q R)} d q,
\end{aligned}
$$

where the third equality comes from $\tilde{V}_{\mathrm{NC}}(q)$ being an odd function of $q,[\ln (q R)=\log (|q| R)$ for $q>0$ and $\ln (q R)=$ $\log (|q| R)+i \pi$ for $q<0$, so that for small $q, \ln (q R) \rightarrow-\infty \approx$ $\ln (|q| R)]$, the 7 th from assuming an upper limit to capture much of the integral, $q_{c} \approx 1 / z$, and the 8 th from the small angle approximation for the sin function.

Last integral in (B.26) may be treated by a normalization $\bar{q}=q / q_{c}$ and a sign absorption from the prefactor:

$$
\begin{aligned}
-\int_{0}^{q_{c}} \frac{1}{\ln (q R)} d q & =-q_{c} \int_{0}^{1} \frac{d \bar{q}}{\ln (\bar{q})+\ln \left(q_{c} R\right)} \\
& =q_{c} \int_{0}^{1} \frac{d \bar{q}}{-\ln (\bar{q})-\ln \left(q_{c} R\right)} \\
& =q_{c} \int_{0}^{1} \frac{d \bar{q}}{a-\ln (\bar{q})} \\
& =q_{c} e^{a} E i(-a) \\
& =q_{c} e^{-\ln \left(q_{c} R\right)} E i\left[\ln \left(q_{c} R\right)\right] .
\end{aligned}
$$

Second to last equality arises from $[64$, see page $523,4.212$, 2 , when $a>0$, and where we have $\left.a=-\ln \left(q_{c} R\right)\right]$. Inserting (B.27) back into (B.26), provides the $V_{\mathrm{NC}}(z)$ solution

$$
\begin{aligned}
& V_{\mathrm{NC}}(z) \\
& \quad \approx \frac{i \rho}{\pi \alpha} I_{c n}\left\{\left\{1+\frac{\delta}{W}\right\}\right\} z q_{c} e^{-\ln \left(q_{c} R\right)} \operatorname{Ei}\left[\ln \left(q_{c} R\right)\right] .
\end{aligned}
$$

For the constant $a$ in the argument of the $E i$ function being a large number ( $z$ is large, $q_{c}$ therefore is tiny), the function may be examined in this limit,

$$
\begin{aligned}
\lim _{a \rightarrow \infty} E i(-a) & =-e^{-a} \int_{0}^{1} \frac{d \bar{q}}{a-\ln (\bar{q})} \\
& \approx-e^{-a} \int_{0}^{1} \frac{d \bar{q}}{a} \\
& =-\frac{e^{-a}}{a} .
\end{aligned}
$$

Placing the (B.29) result into the $V_{\mathrm{NC}}(z)$ formula from (B.28), noting that $z q_{c} \approx 1$,

$$
\left.V_{\mathrm{NC}}(z) \approx-\frac{i \rho}{\pi \alpha} I_{c n}\left\{\left\{1+\frac{\delta}{W}\right\}\right\} \frac{1}{2}\right\} \frac{1}{\ln (R / z)}
$$

which does display the $V_{\mathrm{NC}}(z) \propto 1 / \ln (R / z)$ logarithmic dependence seen by Leonard [14].

\section{References}

[1] H. Scheel, S. Reich, and C. Thomsen, "Electronic band structure of high-index silicon nanowires," Physica Status Solidi B, vol. 242, no. 12, pp. 2474-2479, 2005.

[2] F. Vaurette, J. P. Nys, D. Deresmes, B. Grandidier, and D. Stívenard, "Resistivity and surface states density of n-and p-Type silicon nanowires," Journal of Vacuum Science and Technology B, vol. 26, no. 3, pp. 945-948, 2008.

[3] E. Ramayya, D. Vasileska, S. M. Goodnick, and I. Knezevic, "Electron transport in Si nanowires," Journal of Physics: Conference Series, vol. 38, no. 1, pp. 126-129, 2006.

[4] W. Lu, J. Xiang, B. P. Timko, Y. Wu, and C. M. Lieber, "One-dimensional hole gas in germanium/silicon nanowire heterostructures," Proceedings of the National Academy of Sciences of the United States of America, vol. 102, no. 29, pp. 10046-10051, 2005.

[5] J. W. Mintmire and C. T. White, "Universal density of states for carbon nanotubes," Physical Review Letters, vol. 81, no. 12, pp. 2506-2509, 1998.

[6] P. J. Lin-Chung and A. K. Rajagopal, "Green's function theory of electrical and thermal transport in single-wall carbon nanotubes," Physical Review B, vol. 65, no. 11, Article ID 113408, 4 pages, 2002.

[7] P. M. Martin, "Carbon nanotubes," Vacuum Technology \& Coating, vol. 9, article 8, 2008.

[8] J. W. G. Wildöer, L. C. Venema, A. G. Rinzler, R. E. Smalley, and C. Dekker, "Electronic structure of atomically resolved carbon nanotubes," Nature, vol. 391, no. 6662, pp. 59-62, 1998.

[9] T. W. Odom, J.-L. Huang, P. Kim, and C. M. Lieber, "Atomic structure and electronic properties of single-walled carbon nanotubes," Nature, vol. 391, no. 6662, pp. 62-64, 1998.

[10] F. Léonard and A. A. Talin, "Size-dependent effects on electrical contacts to nanotubes and nanowires," Physical Review Letters, vol. 97, no. 2, Article ID 026804, 4 pages, 2006.

[11] F. Léonard and J. Tersoff, "Novel length scales in nanotube devices," Physical Review Letters, vol. 83, no. 24, pp. 51745177, 1999.

[12] F. Léonard and J. Tersoff, "Role of Fermi-level pinning in nanotube Schottky diodes," Physical Review Letters, vol. 84, no. 20, pp. 4693-4696, 2000. 
[13] M. P. Anantram and F. Léonard, "Physics of carbon nanotube electronic devices," Reports on Progress in Physics, vol. 69, no. 3, pp. 507-561, 2006.

[14] F. Leonard, The Physics of Carbon Nanotube Devices, William Andrew, 2009.

[15] S. O. Koswatta, M. S. Lundstrom, and D. E. Nikonov, "Band-to-band tunneling in a carbon nanotube metal-oxidesemiconductor field-effect transistor is dominated by phononassisted tunneling," Nano Letters, vol. 7, no. 5, pp. 1160-1164, 2007.

[16] P. L. McEuen, M. S. Fuhrer, and H. Park, "Single-walled carbon nanotube electronics," IEEE Transactions on Nanotechnology, vol. 1, no. 1, pp. 78-84, 2002.

[17] C. Kocabas, H.-S. Kim, T. Banks et al., "Radio frequency analog electronics based on carbon nanotube transistors," Proceedings of the National Academy of Sciences of the United States of America, vol. 105, no. 5, pp. 1405-1409, 2008.

[18] J. E. Baumgardner, A. A. Pesetski, J. M. Murduck, J. X. Przybysz, J. D. Adam, and H. Zhang, "Inherent linearity in carbon nanotube field-effect transistors," Applied Physics Letters, vol. 91, no. 5, Article ID 052107, 2007.

[19] A. A. Pesetski, J. E. Baumgardner, E. Folk, J. X. Przybysz, J. D. Adam, and H. Zhang, "Carbon nanotube field-effect transistor operation at microwave frequencies," Applied Physics Letters, vol. 88, no. 11, Article ID 113103, 2006.

[20] C. W. J. Beenakker, “Colloquium: Andreev reflection and Klein tunneling in graphene," Reviews of Modern Physics, vol. 80, no. 4, pp. 1337-1354, 2008.

[21] M. Ishigami, J. H. Chen, W. G. Cullen, M. S. Fuhrer, and E. D. Williams, "Atomic structure of graphene on $\mathrm{SiO}_{2}$," Nano Letters, vol. 7, no. 6, pp. 1643-1648, 2007.

[22] J.-H. Chen, C. Jang, S. Adam, M. S. Fuhrer, E. D. Williams, and M. Ishigami, "Charged-impurity scattering in graphene," Nature Physics, vol. 4, no. 5, pp. 377-381, 2008.

[23] C. Jang, S. Adam, J.-H. Chen, E. D. Williams, S. Das Sarma, and M. S. Fuhrer, "Tuning the effective fine structure constant in graphene: opposing effects of dielectric screening on shortand long-range potential scattering," Physical Review Letters, vol. 101, no. 14, Article ID 146805, 2008.

[24] Y. Ouyang, P. Campbell, and J. Guo, "Analysis of ballistic monolayer and bilayer graphene field-effect transistors," Applied Physics Letters, vol. 92, no. 6, Article ID 063120, 2008.

[25] J.-H. Chen, C. Jang, S. Xiao, M. Ishigami, and M. S. Fuhrer, "Intrinsic and extrinsic performance limits of graphene devices on $\mathrm{SiO}_{2}$," Nature Nanotechnology, vol. 3, no. 4, pp. 206-209, 2008.

[26] J.-H. Chen, M. Ishigami, C. Jang, D. R. Hines, M. S. Fuhrer, and E. D. Williams, "Printed graphene circuits," Advanced Materials, vol. 19, no. 21, pp. 3623-3627, 2007.

[27] D. Jena, T. Fang, Q. Zhang, and H. Xing, "Zener tunneling in semiconducting nanotube and graphene nanoribbon $\mathrm{p}-\mathrm{n}$ junctions," Applied Physics Letters, vol. 93, no. 11, Article ID 112106, 2008.

[28] R. M. Westervelt, "Graphene nanoelectronic," Science, vol. 320, article 324, 2008.

[29] P. Zhao, J. Chauhan, and J. Guo, "Computational study of tunneling transistor based on graphene nanoribbon," Nano Letters, vol. 9, no. 2, pp. 684-688, 2009.

[30] C. N. Chervin, A. M. Lubers, K. A. Pettigrew et al., "Making the most of a scarce platinum-group metal: conductive ruthenia nanoskins on insulating silica paper," Nano Letters, vol. 9, no. 6, pp. 2316-2321, 2009.
[31] D. R. Rolison, J. W. Long, J. C. Lytle et al., "Multifunctional 3D nanoarchitectures for energy storage and conversion," Chemical Society Reviews, vol. 38, no. 1, pp. 226-252, 2009.

[32] D. M. Adams, L. Brus, C. E. D. Chidsey et al., "Charge transfer on the nanoscale: current status," Journal of Physical Chemistry B, vol. 107, no. 28, pp. 6668-6697, 2003.

[33] A. J. McEvoy and W. Gissler, "A ruthenium dioxidesemiconductor Schottky barrier photovoltaic device," Journal of Applied Physics, vol. 53, no. 2, pp. 1251-1252, 1982.

[34] C. S. Hsieh, G. Wang, D. S. Tsai, R. S. Chen, and Y. S. Huang, "Field emission characteristics of ruthenium dioxide nanorods," Nanotechnology, vol. 16, no. 9, pp. 1885-1891, 2005.

[35] M. D. Smolin, V. N. Fedorov, V. G. Grebenkina, G. M. Artamonov, and Yu. A. Kornatovskii, "Influence of the degree of dispersion of ruthenium dioxide on the electrical properties of thick-film ruthenium resistors," Poroshkovaya Metallurgiya, vol. 6, no. 294, pp. 71-75, 1987, translation in Soviet Metallurgy Ceramics vol. 26, pp. 495-499, 1987.

[36] L. M. Rossi, J. Dupont, G. Machado et al., "Ruthenium dioxide nanoparticles in ionic liquids: synthesis, characterization and catalytic properties in hydrogenation of olefins and arenes," Journal of the Brazilian Chemical Society, vol. 15, no. 6, pp. 904-910, 2004.

[37] H. Over, "Ruthenium dioxide, a fascinating material for atomic scale surface chemistry," Applied Physics A, vol. 75, no. 1, pp. 37-44, 2002.

[38] S. I. Maximenko, L. Mazeina, Y. N. Picard, J. A. Freitas, V. M. Bermudez, and S. M. Prokes, "Cathodoluminescence studies of the inhomogeneities in sn-doped $\mathrm{Ga}_{2} \mathrm{O}_{3}$ nanowires," Nano Letters, vol. 9, no. 9, pp. 3245-3251, 2009.

[39] V. M. Bermudez, "The structure of low-index surfaces of $\beta$ $\mathrm{Ga}_{2} \mathrm{O}_{3}$," Chemical Physics, vol. 323, no. 2-3, pp. 193-203, 2006.

[40] M. Passlack, E. F. Schubert, W. S. Hobson et al., " $\mathrm{Ga}_{2} \mathrm{O}_{3}$ films for electronic and optoelectronic applications," Journal of Applied Physics, vol. 77, no. 2, pp. 686-693, 1995.

[41] B. Liu, M. Gu, and X. Liu, "Lattice dynamical, dielectric, and thermodynamic properties of $\beta-\mathrm{Ga}_{2} \mathrm{O}_{3}$ from first principles," Applied Physics Letters, vol. 91, no. 17, Article ID 172102, 2007.

[42] G. W. Paterson, J. A. Wilson, D. Moran et al., "Gallium oxide $\left(\mathrm{Ga}_{2} \mathrm{O}_{3}\right)$ on gallium arsenide-A low defect, high-K system for future devices," Materials Science and Engineering B, vol. 135, no. 3, pp. 277-281, 2006.

[43] J. Liu and J. P. Coleman, "Nanostructured metal oxides for printed electrochromic displays," Materials Science and Engineering A, vol. 286, no. 1, pp. 144-148, 2000.

[44] Y. Zhang, S. H. Lee, A. Mascarenhas, and S. K. Deb, "An UV photochromic memory effect in proton-based $\mathrm{WO}_{3}$ electrochromic devices," Applied Physics Letters, vol. 93, no. 20, Article ID 203508, 2008.

[45] G. Gu, B. Zheng, W. Q. Han, S. Roth, and J. Liu, "Tungsten oxide nanowires on tungsten substrates," Nano Letters, vol. 2, no. 8, pp. 849-851, 2002.

[46] M. E. Franke, T. J. Koplin, and U. Simon, "Metal and metal oxide nanoparticles in chemiresistors: does the nanoscale matter?" Small, vol. 2, no. 1, pp. 36-50, 2006.

[47] O. N. Mryasov and A. J. Freeman, "Electronic band structure of indium tin oxide and criteria for transparent conducting behavior," Physical Review B, vol. 64, no. 23, Article ID 233111, 3 pages, 2001.

[48] H. Kim, C. M. Gilmore, A. Piqué et al., "Electrical, optical, and structural properties of indium-tin-oxide thin films for 
organic light-emitting devices," Journal of Applied Physics, vol. 86, no. 11, pp. 6451-6461, 1999.

[49] C. Laberty-Robert, J. W. Long, K. A. Pettigrew, R. M. Stroud, and D. R. Rolison, "Ionic nanowires at $600{ }^{\circ} \mathrm{C}$ : using nanoarchitecture to optimize electrical transport in nanocrystalline gadolinium-doped ceria," Advanced Materials, vol. 19, no. 13, pp. 1734-1739, 2007.

[50] W. H. Chang, C. H. Lee, P. Chang et al., "High $\kappa$ dielectric single-crystal monoclinic $\mathrm{Gd}_{2} \mathrm{O}_{3}$ on $\mathrm{GaN}$ with excellent thermal, structural, and electrical properties," Journal of Crystal Growth, vol. 311, no. 7, pp. 2183-2186, 2009.

[51] J. Wei, Z. Wang, W. Chen, and D. H. Cobden, "New aspects of the metal-insulator transition in single-domain vanadium dioxide nanobeams," Nature Nanotechnology, vol. 4, no. 7, pp. 420-424, 2009.

[52] G. L. Zhao, D. Bagayoko, and T. D. Williams, "Local-densityapproximation prediction of electronic properties of $\mathrm{GaN}, \mathrm{Si}$, C, and $\mathrm{RuO}_{2}$," Physical Review B, vol. 60, no. 3, pp. 1563-1572, 1999.

[53] K. Benyahia, Z. Nabi, A. Tadjer, and A. Khalf, "Ab initio study of the structural and electronic properties of the complex structures of $\mathrm{RuO}_{2}$," Physica B, vol. 339, no. 1, pp. 1-10, 2003.

[54] U. Lundin, L. Fast, L. Nordström, B. Johansson, J. M. Wills, and O. Eriksson, "Transition-metal dioxides with a bulk modulus comparable to diamond," Physical Review B, vol. 57, no. 9, pp. 4979-4982, 1998.

[55] K. M. Glassford and J. R. Chelikowsky, "Electron transport properties in $\mathrm{RuO}_{2}$ rutile," Physical Review B, vol. 49, no. 11, pp. 7107-7114, 1994.

[56] K. M. Glassford and J. R. Chelikowsky, "Electronic and structural properties of $\mathrm{RuO}_{2}$," Physical Review B, vol. 47, no. 4, pp. 1732-1741, 1993.

[57] O. V. Krasovska, E. E. Krasovskii, and V. N. Antonov, "Ab initio calculation of the optical and photoelectron properties of $\mathrm{RuO}_{2}$," Physical Review B, vol. 52, no. 16, pp. 11825-11829, 1995.

[58] J. H. Xu, T. Jarlborg, and A. J. Freeman, "Self-consistent band structure of the rutile dioxides $\mathrm{NbO}_{2}, \mathrm{RuO}_{2}$, and $\mathrm{IrO}_{2}$," Physical Review B, vol. 40, no. 11, pp. 7939-7947, 1989.

[59] K. V. K. Rao and L. Iyengar, "X-ray studies on the thermal expansion of ruthenium dioxide," Acta Crystallographica, vol. A25, pp. 302-303, 1969.

[60] S. Ramo, J. R. Whinnery, and T. van Duzer, Fields and Waves in Communication Electronics, John Wiley \& Sons, New York, NY, USA, 1967.

[61] W. D. Ryden, A. W. Lawson, and C. C. Sartain, "Electrical transport properties of $\mathrm{IrO}_{2}$ and $\mathrm{RuO}_{2}$," Physical Review B, vol. 1, no. 4, pp. 1494-1500, 1970.

[62] E. M. Purcell, Electricity and Magnetism, vol. 2 of Berkeley Physics Course, McGraw-Hill, New York, NY, USA, 1965.

[63] G. Arfken, Mathematical Methods for Physicists, Academic Press, Amsterdam, The Netherlands, 1968.

[64] I. S. Gradshteyn and I. M. Ryzhik, Table of Integrals, Series, and Products, Academic Press, 1980.

[65] S. M. Sze, Physics of Semiconductor Devices, John Wiley \& Sons, New York, NY, USA, 1981.

[66] C. M. Krowne and R. E. Neidert, "Theory and numerical calculations for radially inhomogeneous circular ferrite circulators," IEEE Transactions on Microwave Theory and Techniques, vol. 44, no. 3, pp. 419-431, 1996.

[67] C. M. Krowne, “Theory of the recursive dyadic Green's function for inhomogeneous ferrite canonically Sshaped microstrip circulators," in Advances in Imaging and Electron
Physics, P. W. Hawkes, Ed., vol. 98, pp. 77-321, Academic Press, Edinburg, UK, 1996.

[68] H. S. Newman and C. M. Krowne, "Analysis of ferrite circulators by 2 -d finite-element and recursive Green's function techniques," IEEE Transactions on Microwave Theory and Techniques, vol. 46, no. 2, pp. 167-177, 1998.

[69] C. M. Krowne, “Dyadic Green's function microstrip circulator theory for inhomogeneous ferrite with and without penetrable walls," in Advances in Imaging and Electron Physics, P. W. Hawkes, Ed., vol. 103, pp. 151-275, Academic Press, Amsterdam, The Netherlands, 1998.

[70] C. M. Krowne, “Implicit 3-D dyadic Green's function using self-adjoint operators for inhomogeneous planar ferrite circulator with vertically layered external material employing mode-matching," IEEE Transactions on Microwave Theory and Techniques, vol. 46, no. 4, pp. 359-377, 1998.

[71] C. M. Krowne, "Anisotropic recursive dyadic Green's function 3D theory for a radially inhomogeneous microstrip circular ferrite circulator," International Journal of Numerical Modelling: Electronic Networks, Devices and Fields, vol. 12, no. 5, pp. 355-387, 1999.

[72] C. M. Herzinger, B. Johs, W. A. McGahan, J. A. Woollam, and W. Paulson, "Ellipsometric determination of optical constants for silicon and thermally grown silicon dioxide via a multisample, multi-wavelength, multi-angle investigation," Journal of Applied Physics, vol. 83, no. 6, pp. 3323-3336, 1998.

[73] P. Hones, T. Gerfin, and M. Grätzel, "Spectroscopic ellipsometry of $\mathrm{RuO}_{2}$ films prepared by metalorganic chemical vapor deposition," Applied Physics Letters, vol. 67, article 3078, 1995.

[74] G. Mondio, F. Neri, M. Allegrini, A. Lembo, and F. Fuso, "Energy loss spectroscopy of $\mathrm{RuO}_{2}$ thin films," Journal of Applied Physics, vol. 82, no. 4, pp. 1730-1735, 1997.

[75] C. M. Krowne, "Multi-species two-level atomic media displaying electromagnetic left handedness," Physics Letters A, vol. 372, no. 13, pp. 2304-2310, 2008.

[76] H. B. Dwight, Table of Integrals and Other Mathematical Data, Macmillan, New York, NY, USA, 1961.

[77] P. M. Morse and H. Feshbach, Methods of Theoretical Physics, McGraw-Hill, New York, NY, USA, 1953.

[78] M. N. Jones, Y. W. Kwon, and D. P. Norton, "Dielectric constant and current transport for $\mathrm{HfO}_{2}$ thin films on ITO," Applied Physics A, vol. 81, no. 2, pp. 285-288, 2005.

[79] H. J. Wadsworth, S. Bhattacharya, D. W. McNeill et al., "Germanium MOS capacitors with hafnium dioxide and silicon dioxide dielectrics," Materials Science in Semiconductor Processing, vol. 9, no. 4-5, pp. 685-689, 2006.

[80] X. Liu, S. Ramanathan, and T. E. Seidel, "Atomic layer deposition of hafnium oxide thin films from tetrakis(dimethylamino)hafnium (TDMAH) and ozone," Materials Research Society Symposium Proceedings, vol. 765, pp. 97-102, 2003.

[81] X. Garros, C. Leroux, D. Blin, J. F. Damlencourt, A. M. Papon, and G. Reimbold, "Investigation of $\mathrm{HfO}_{2}$ dielectric stacks deposited by ALD with a mercury probe," in Proceedings of the 39th European Solid-State Device Research Conference (ESSDERC'02), p. 411, 2002.

[82] W. C. Lee, B. H. Chin, L. K. Chu et al., "Molecular beam epitaxy-grown $\mathrm{Al}_{2} \mathrm{O}_{3} / \mathrm{HfO}_{2}$ high- $\kappa$ dielectrics for germanium," Journal of Crystal Growth, vol. 311, no. 7, pp. 21872190, 2009.

[83] J. P. Zheng and T. R. Jow, "High energy and high power density electrochemical capacitors," Journal of Power Sources, vol. 62, no. 2, pp. 155-159, 1996. 
[84] B. E. Conway, V. Birss, and J. Wojtowicz, "The role and utilization of pseudocapacitance for energy storage by supercapacitors," Journal of Power Sources, vol. 66, no. 1-2, pp. 1-14, 1997.

[85] C. M. Krowne, "Intrinsic quantum conductances and capacitances of nanowires and nanocables," Physics Letters A, vol. 374, no. 4, pp. 614-619, 2010.

[86] C. M. Krowne, "Nanowire and nanocable junction capacitances: results for metal and semiconducting oxides," Physics Letters A, vol. 374, no. 9, pp. 1172-1179, 2010.

[87] I. S. Bayer and A. Biswas, "Ceramic catalysts for the growth of single wall carbon nanotubes (SWCNTs)," Vacuum Technology \& Coating, vol. 10, article 26, 2009.

[88] X. Zhou, T. Zifer, B. M. Wong, K. L. Krafcik, F. Léonard, and A. L. Vance, "Color detection using chromophore-nanotube hybrid devices," Nano Letters, vol. 9, no. 3, pp. 1028-1033, 2009.

[89] T. S. Kang, A. P. Smith, B. E. Taylor, and M. F. Durstock, "Fabrication of highly-ordered $\mathrm{TiO}_{2}$ nanotube arrays and their use in dye-sensitized solar cells," Nano Letters, vol. 9, no. 2, pp. 601-606, 2009.

[90] Q. Wang, K. Zhu, N. R. Neale, and A. J. Frank, "Constructing ordered sensitized heterojunctions: bottom-up electrochemical synthesis of p-type semiconductors in oriented $\mathrm{n}-\mathrm{TiO}_{2}$ nanotube arrays," Nano Letters, vol. 9, no. 2, pp. 806-813, 2009.

[91] S. W. Lee, N. Yabuuchi, B. M. Gallant et al., "High-power lithium batteries from functionalized carbon-nanotube electrodes," Nature Nanotechnology, vol. 5, no. 7, pp. 531-537, 2010.

[92] L. Mai, Y. Gu, C. Han et al., "Orientated langmuir-blodgett assembly of $\mathrm{VO}_{2}$ nanowires," Nano Letters, vol. 9, no. 2, pp. 826-830, 2009.

[93] J. Huang, W. K. Chim, S. Wang, S. Y. Chiam, and L. M. Wong, "From germanium nanowires to germanium-silicon oxide nanotubes: influence of germanium tetraiodide precursor," Nano Letters, vol. 9, no. 2, pp. 583-589, 2009.

[94] L. Mai, Y. Dong, L. Xu, and C. Han, "Single nanowire electrochemical devices," Nano Letters, vol. 10, no. 10, pp. 4273-4278, 2010.

[95] Y. Zhang, L. W. Wang, and A. Mascarenhas, "'Quantum coaxial cables" for solar energy harvesting," Nano Letters, vol. 7, no. 5, pp. 1264-1269, 2007.

[96] M. Kaempgen, C. K. Chan, J. Ma, Y. Cui, and G. Gruner, "Printable thin film supercapacitors using single-walled carbon nanotubes," Nano Letters, vol. 9, no. 5, pp. 1872-1876, 2009.

[97] B. P. Lathi, Signals, Systems and Communication, John Wiley \& Sons, New York, NY, USA, 1965.

[98] M. Abramowitz and I. A. Stegun, Handbook of Mathematical Functions with Formulas, Graphs, and Mathematical Tables, vol. 55 of National Bureau of Standards, Applied Matematics Series, 10th edition, 1972.

[99] R. F. Harrington, Time-Harmonic Electromagnetic Fields, McGraw-Hill, New York, NY, USA, 1961. 

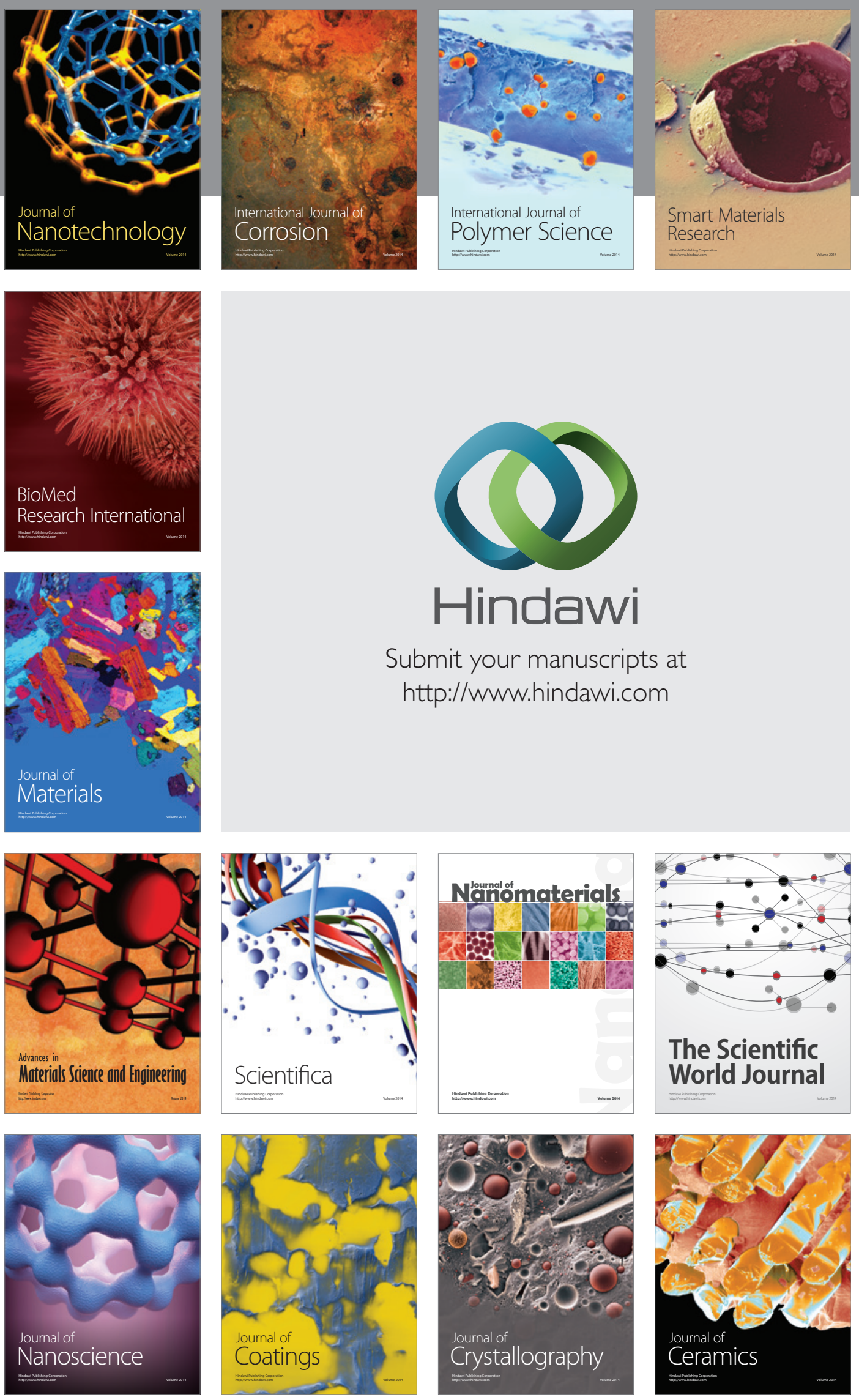

The Scientific World Journal

Submit your manuscripts at

http://www.hindawi.com

\section{World Journal}

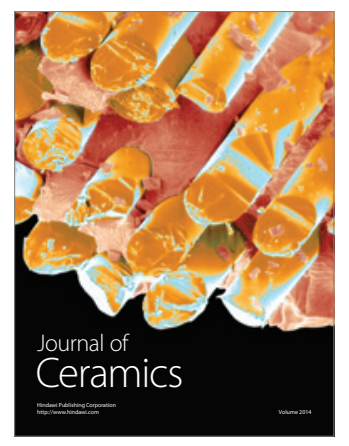

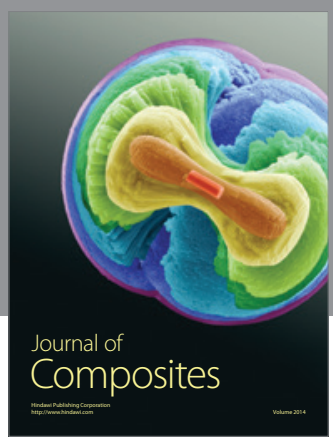
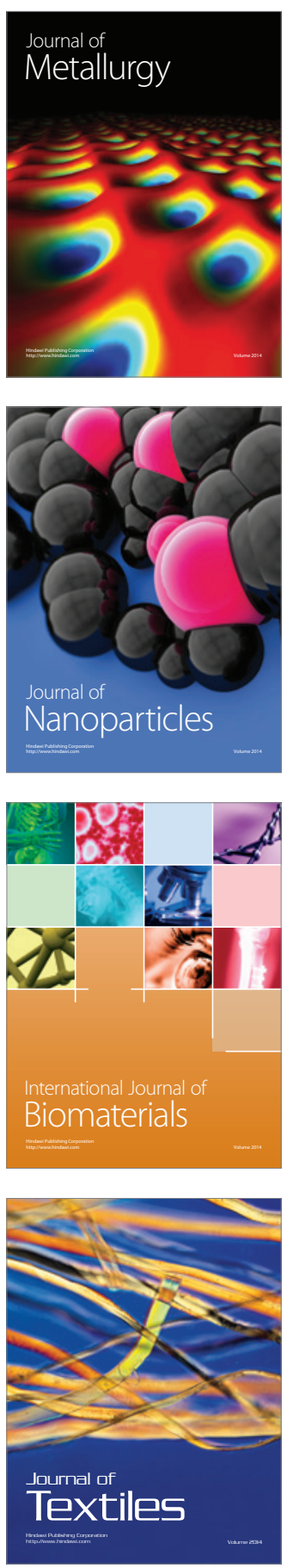